\title{
Use of Streamline Analysis Method to Model Aerodynamic Forces in Axial Compressors
}

\author{
A Thesis \\ Presented to \\ the faculty of the School of Engineering and Applied Science \\ University of Virginia
}

\author{
In Partial Fulfillment \\ of the requirements for the Degree \\ Master of Science
}

by

Ami Nalin Patel

May 2013 


\begin{abstract}
APPROVAL SHEET
The thesis is submitted in partial fulfillment of the requirements for the degree of

Master of Science
\end{abstract}

Ami N. Patel, Author

This thesis has been read and approved by the examining Committee:

Robert J. Ribando, Advisor

Houston G. Wood, Committee Chair

Christopher P. Goyne, Committee Member

Eric Loth, Committee Member

Accepted for the School of Engineering and Applied Science:

James H. Aylor, Dean

School of Engineering and Applied Science

May 2013 


\section{Abstract}

Within the field of rotor dynamics, the aerodynamic loading on turbomachinery blades is an important factor for calculating the overall stability of these machines. In order to determine the magnitude of this force, it is necessary to analyze the flow of the fluid through the turbomachinery. With respect to modern jet engines, an axial compressor is the component through which the incoming air passes first. As the performance of the compressor often determines the efficiency of the overall engine, it is crucial to analyze and understand the fluid effects in the compressor properly. Therefore, the goal of this thesis is to develop a tool for determining the aerodynamic forces on the blades of axial compressors in jet engines for further use in rotor dynamic calculations.

To avoid a time consuming and computationally expensive procedure, a twodimensional "streamline" or "through-flow" analysis is adopted, in which various assumptions are used to simplify the Navier-Stokes equations. For the streamline model, the flow between blades is divided into "annuli", and fluid properties are calculated from hub to casing while marching axially downstream through the multiple stages making up the compressor. The streamline analysis method was coded in Matlab as a software package called CompFlow. Computational results are then compared with experimental values available in literature. Cases studied include a single-stage rotor case called Stage 37, and a multistage compressors case called NASA 74A. A case study was also conducted on the GEnx-2B, a current jet engine consisting of a low-pressure compressor and high-pressure compressor that was developed by GE Aviation. 


\section{Acknowledgments}

I would first like to thank my advisor, Bob Ribando, who gave me guidance during the course of my project. Also, I would like to thank the ROMAC research group at the University of Virginia for providing financial support for me as a Graduate Research Assistant for three semesters and providing the chance to pursue a Master's degree. Also I'd like to thank the department for supporting me as a Graduate Teaching Assistant for three semesters. I would especially like to thank Pratt and Whitney for granting me the opportunity to co-op with them, which allowed me to obtain valuable insight towards my project.

To my colleagues ROMAC, thank you for answering all my frantic questions and providing moral support. To my friends in and out of grad school, this process wouldn't have been the same without your amazing friendships and the many wonderful memories I've gained. I'd especially like to thank my roommate April for making sure that I ate properly while I was working on my thesis.

This thesis would not be possible without my family. I want to thank my parents and godparents for their quiet support and constant faith in my abilities. Much gratitude goes to my best friends JB and Caroline, for always being there for me. And finally, many thanks to my sister Rita for making me laugh every day, especially when I needed it the most.

odi et amo. quare id faciam fortasse requires?

nescio, sed fieri sentio et excrucior.

$\sim$ Catullus 


\section{Table of Contents}

\section{Chapter 1: Introduction ..................................................................................1}

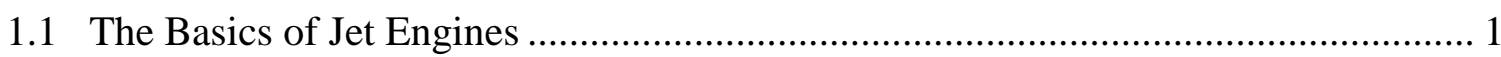

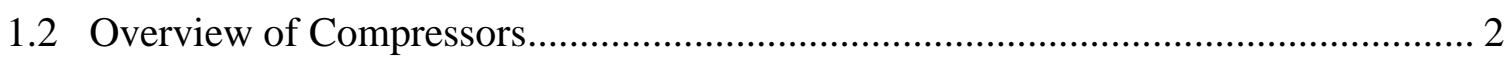

1.3 Fluid Dynamics of Compressors.......................................................................... 3

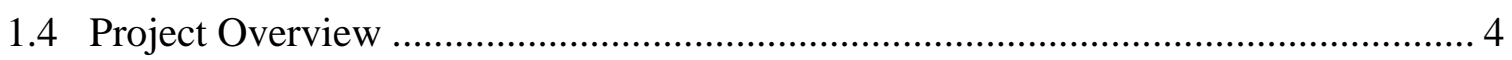

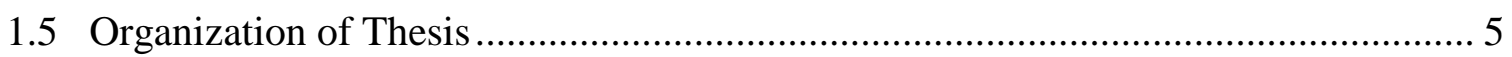

\section{Chapter 2: Literature Research ..................................................................7}

2.1 Overview of Mathematical Models .................................................................. 7

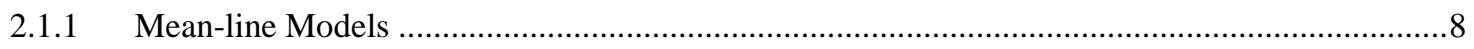

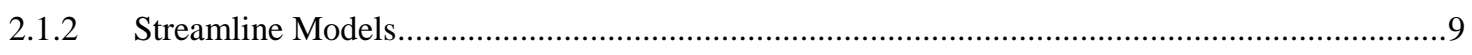

2.1.3 CFD . .

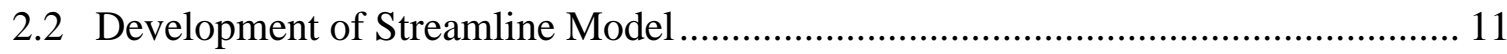

\section{Chapter 3: CompFlow Methodology ......................................................................13}

3.1 Introduction to CompFlow ............................................................................. 13

3.2 Assumptions .............................................................................................. 14

3.2.1 Derivation of Simple Radial Equilibrium Equation ..............................................................15

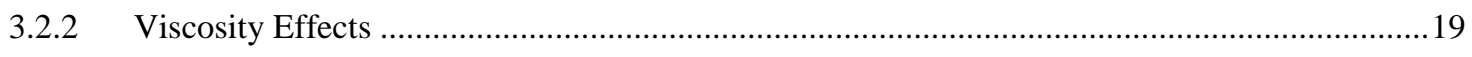

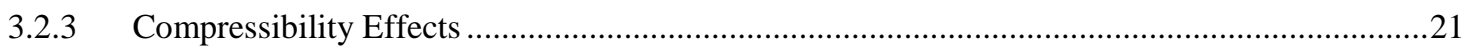

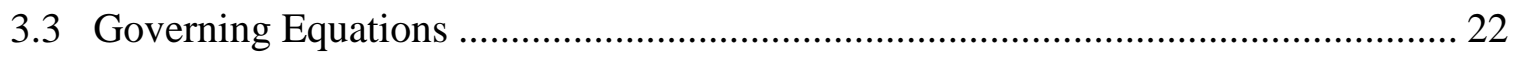

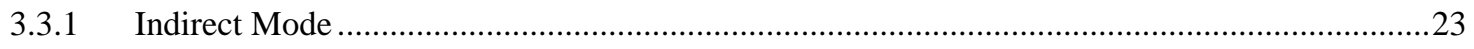

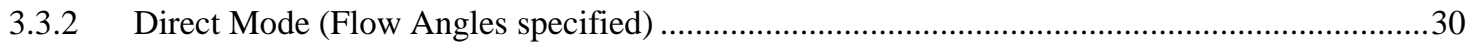

3.4 Post-Processing Calculations …………………………………………………... 33 
3.4.1 Overall Pressure and Temperature Ratios ...............................................................................3

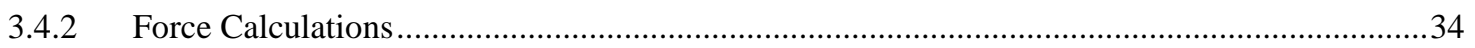

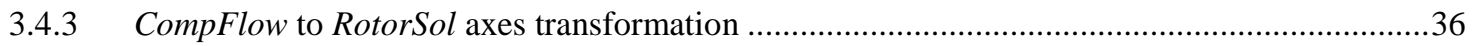

\section{Chapter 4: Verification and Validation...................................................38}

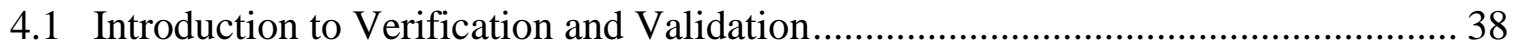

4.2 Verification using Flack's Single Stage Compressor ......................................... 38

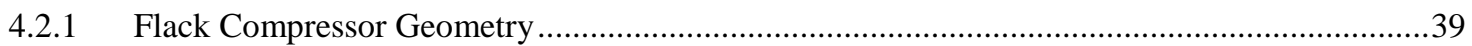

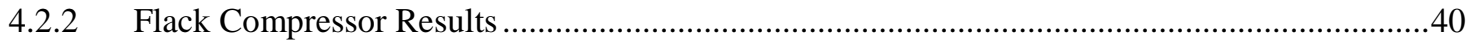

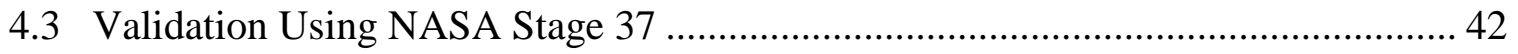

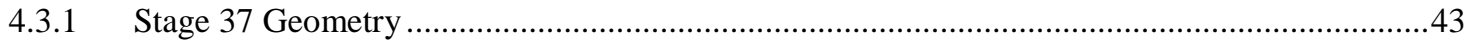

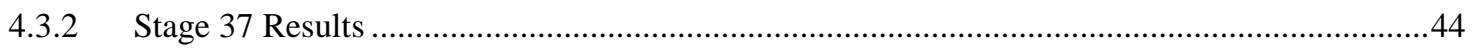

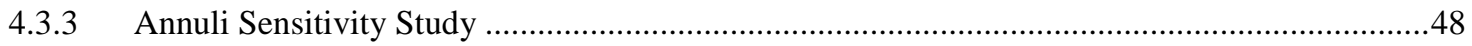

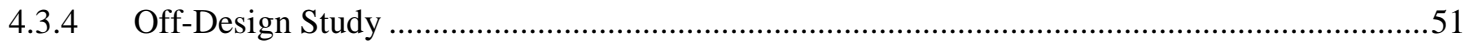

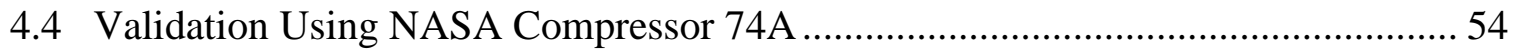

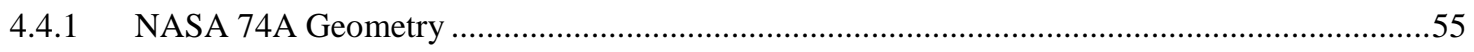

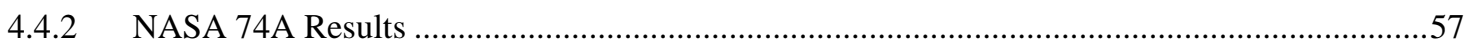

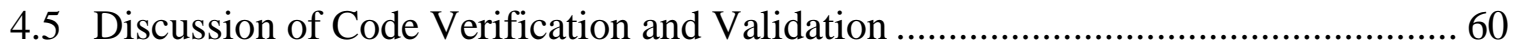

Chapter 5: GEnx-2B Case Study ......................................................................62

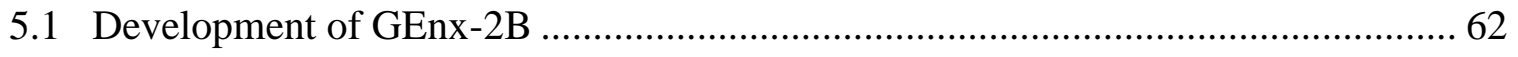

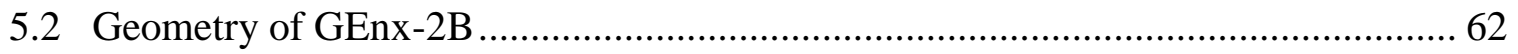

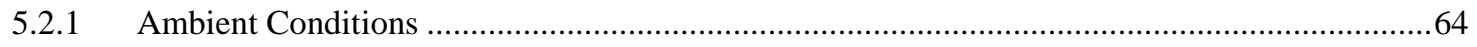

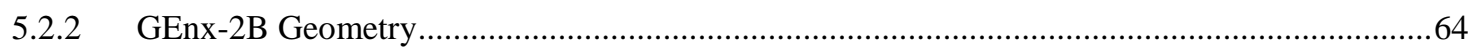

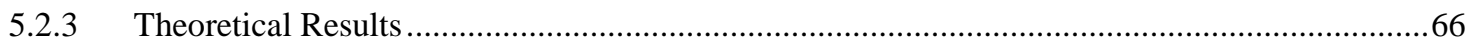

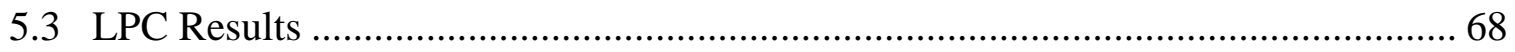

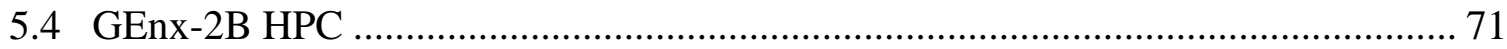

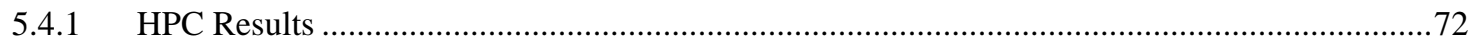


Chapter 6: Conclusions ......................................................................................74

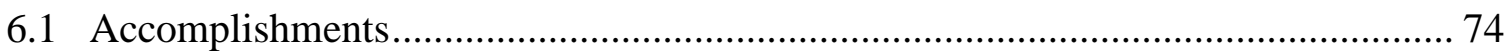

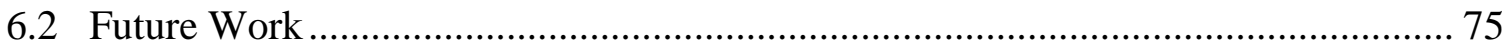

Appendix A: Density Calculations ............................................................80

Appendix B: Summary of Equations .......................................................81

Appendix C: CompFlow GUI User Guide ................................................86 


\section{List of Figures}

Figure 1.1: Schematic showing main components of high-bypass ratio turbofan [3] ....... 2

Figure 2.1: Range of Mathematical Models. [8] ...................................................... 7

Figure 2.2: Example of mean-line geometry for a single stage [10] ............................ 9

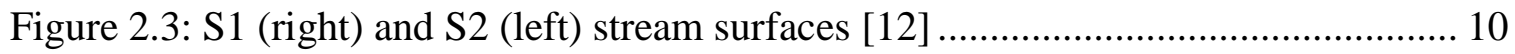

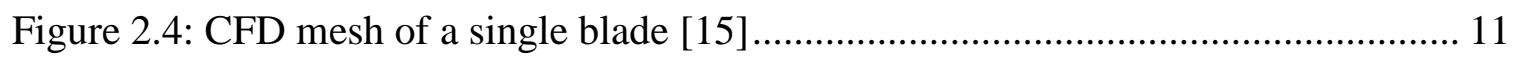

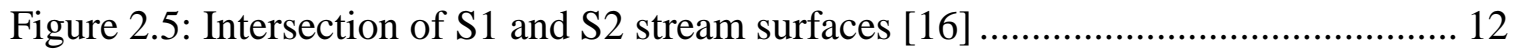

Figure 3.1: Depiction of annulus and axial station positions ..................................... 13

Figure 3.2: Wedge of radial fluid for radial equilibrium derivation [5] ........................ 16

Figure 3.3: Boundary layers and wakes along the surface of stator blades [5] ............... 20

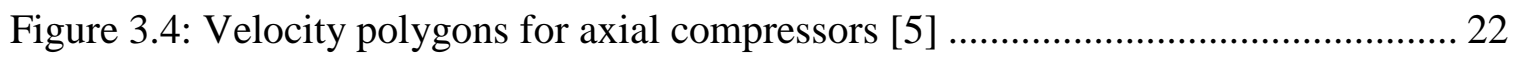

Figure 3.5: Flowchart for indirect mode calculations ............................................. 29

Figure 3.6: Flowchart for direct mode calculations ............................................... 32

Figure 3.7: Control volume for force component calculations ................................. 35

Figure 3.8: RotorSol and CompFlow (red) coordinate systems [22]............................ 37

Figure 4.1: Geometry for Flack's single stage compressor [5] .................................. 39

Figure 4.2: Axial Velocity comparison for CompFlow (a) and Flack (b) [5].................. 41

Figure 4.3: Stage 37 results for axial velocity (a) and Mach number (b) ...................... 45

Figure 4.4: Stage 37 results for total pressure ratio (a) and total temperature ratio (b) .... 45

Figure 4.5: Stage 37 results for blade force (a) and streamlines (b) ............................ 46

Figure 4.6: Comparison with experimental data for Stage 37 ................................ 47 
Figure 4.7: Results for Stage 37 sensitivity study with (a) 11 annuli, (b) 9 annuli, (c) 7

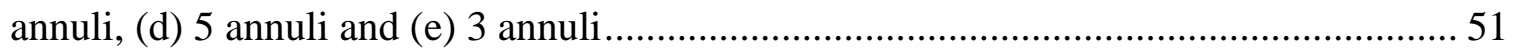

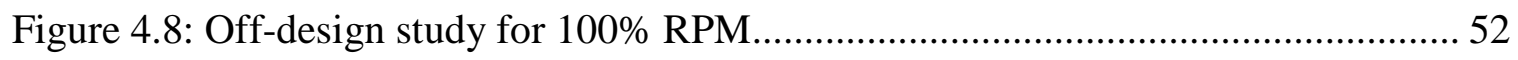

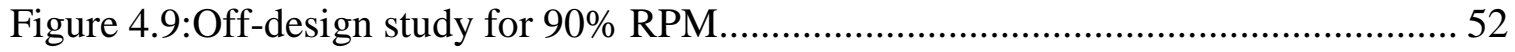

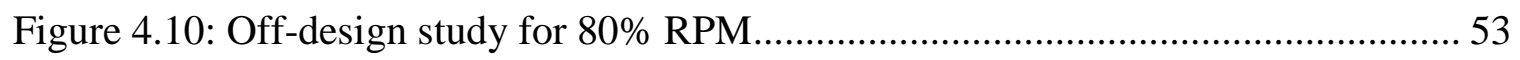

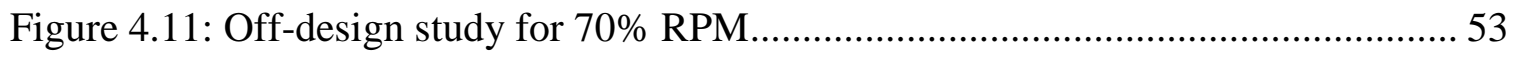

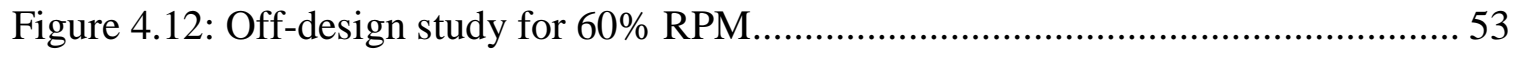

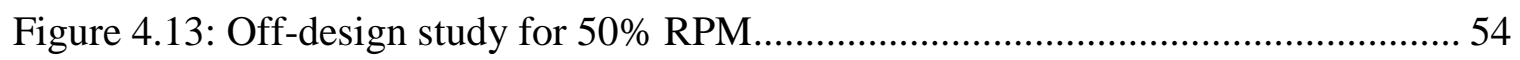

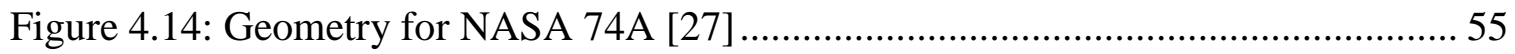

Figure 4.15:NASA 74A results for axial velocity (a) and Mach number (b) ................. 57

Figure 4.16: NASA 74A results for total pressure ratio (a) and total temperature ratio (b) 58

Figure 4.17: NASA 74A results for total blade force (a) and streamline (b) ................... 58

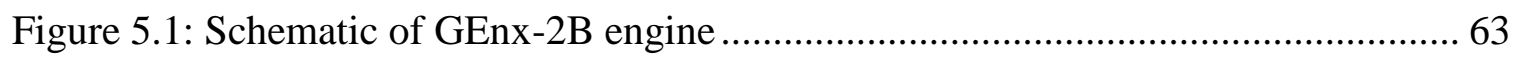

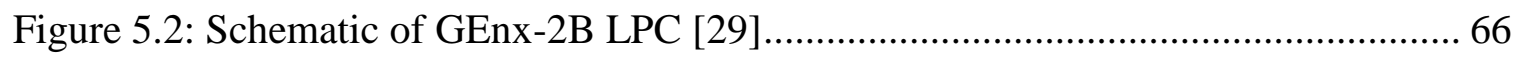

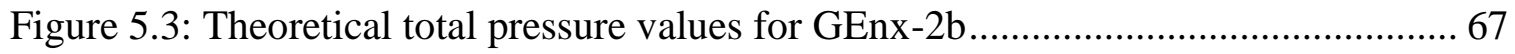

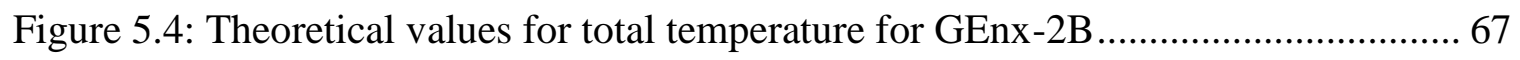

Figure 5.5: LPC distributions for (a) axial velocity and (b) Mach Number .................... 68

Figure 5.6: LPC results for (a) blade force distribution and (b) streamline movement... 69

Figure 5.7: Comparison of theoretical total pressures to CompFlow results .................. 70

Figure 5.8: Comparison of theoretical total temperatures to CompFlow results ............. 70

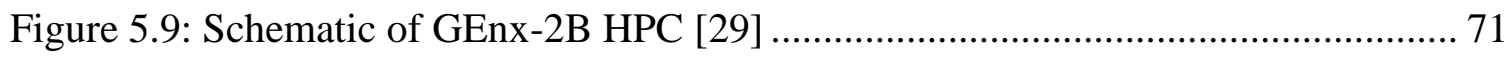

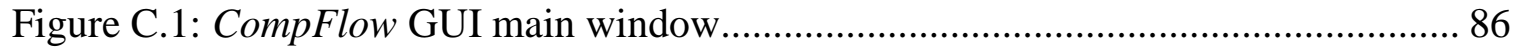




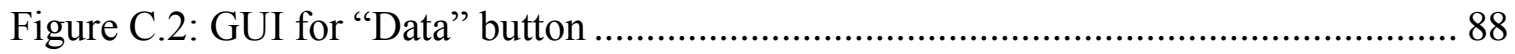

Figure C.3: Window for selecting .mat file using "Calculations" option in main window

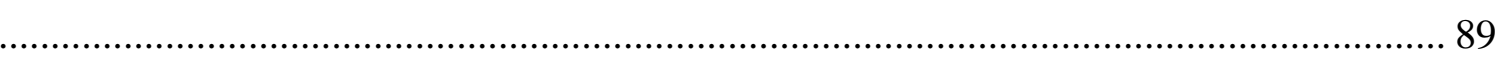

Figure C.4: Example of using "Calculations" option in main window ............................. 90

Figure C.5: Window for plotting results from CompFlow ………………………........ 92 


\section{List of Tables}

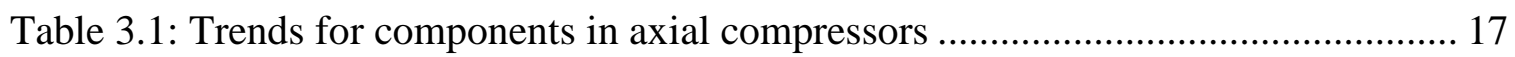

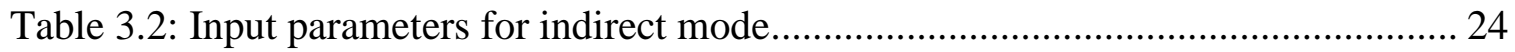

Table 3.3: Flow of Calculations for Indirect Method ................................................. 28

Table 3.4: Flow of Calculations for Direct Mode.......................................................... 31

Table 4.1: Input parameters for Flack compressor .................................................... 40

Table 4.2: Comparison of static and total pressures for Flack compressor ..................... 42

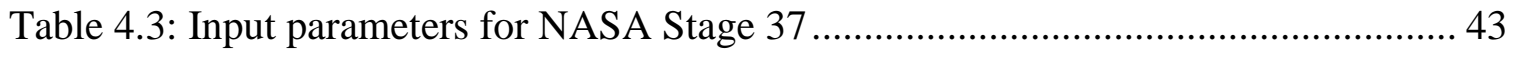

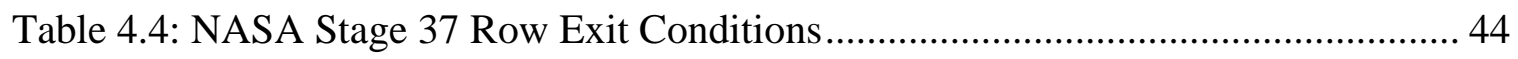

Table 4.5: Comparison of Overall Compressor Data............................................... 48

Table 4.6: Rotor Exit Flow Angles for Senstivity Study ......................................... 49

Table 4.7: Efficiency Distributions for Sensitivity Study ........................................ 49

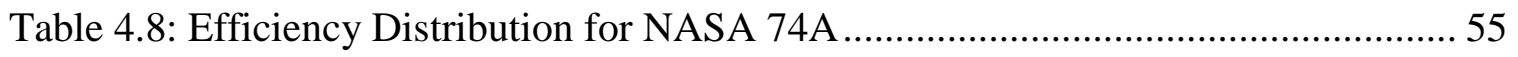

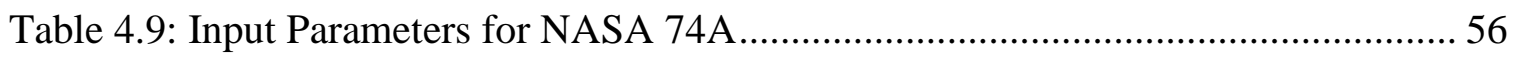

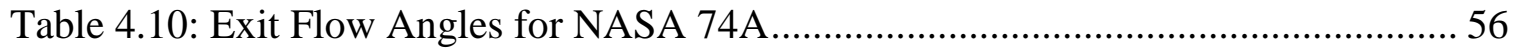

Table 4.11: Comparison of computational and experimental data for NASA74A.......... 59

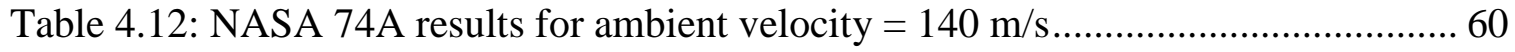

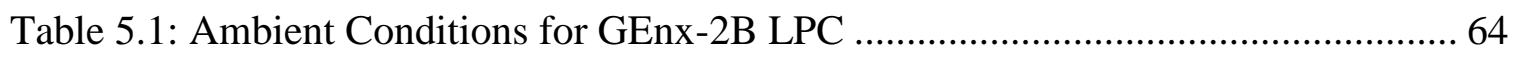

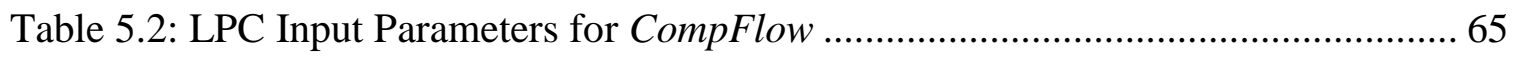

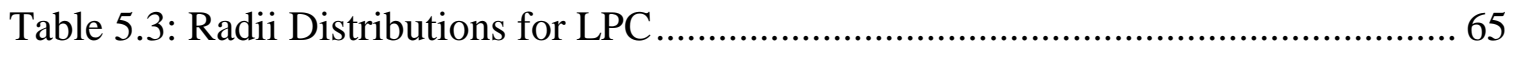

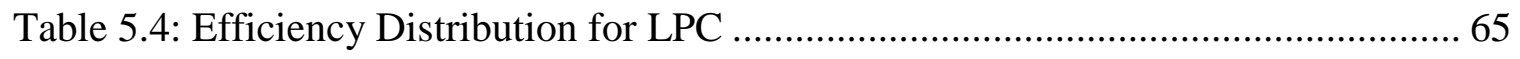

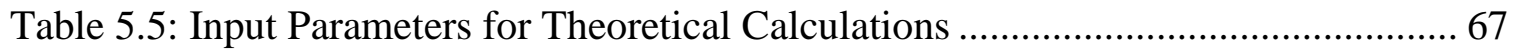




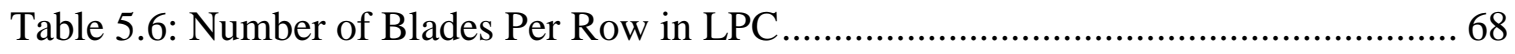

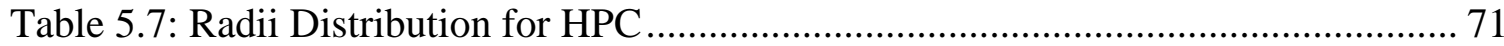

Table 5.8: HPC Input Parameters for CompFlow .................................................. 72

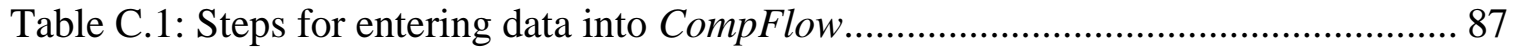

Table C.2: Steps for plotting results in CompFlow................................................. 92 


\section{Nomenclature}

\begin{tabular}{|c|c|}
\hline 2D & Two dimensional \\
\hline 3D & Three dimensional \\
\hline $\mathbf{a}$ & Speed of sound \\
\hline $\mathbf{A}$ & Area \\
\hline c & Absolute velocity component \\
\hline CFD & Computational Fluid Dynamics \\
\hline $\mathbf{F}$ & Force component \\
\hline h & Height of blades in a row \\
\hline HPC & High-pressure compressor \\
\hline IGV & Inlet guide vane \\
\hline LPC & Low-pressure compressor \\
\hline $\mathbf{M}$ & Mach number \\
\hline$\dot{\mathbf{m}}$ & Mass flow rate \\
\hline $\mathbf{M}_{\mathbf{0}}$ & Moment in the tangential direction \\
\hline $\mathbf{p}$ & Static pressure \\
\hline PR & Pressure ratio across a blade row \\
\hline $\mathbf{P t}$ & Total (stagnation) pressure \\
\hline $\mathbf{Q}$ & Volumetric flow rate \\
\hline $\mathbf{R}$ & Radius of the blade \\
\hline $\mathbf{r}$ & Radial axis for CompFlow \\
\hline ROMAC & Rotating machinery \\
\hline $\mathbf{S}$ & Spacing between blades in a row \\
\hline S1 & Stream surface describing blade-to-blade flow \\
\hline $\mathbf{S 2}$ & Stream surface describing hub-to-tip flow \\
\hline $\mathbf{T p}$ & Static temperature \\
\hline $\mathbf{T}_{\mathbf{q}}$ & Torque \\
\hline TR & Temperature ratio across a blade row \\
\hline $\mathbf{T t}$ & Total (stagnation) temperature \\
\hline $\mathbf{U}$ & Blade velocity \\
\hline $\mathbf{w}$ & Relative velocity component \\
\hline$\dot{\mathbf{W}}$ & Power \\
\hline
\end{tabular}


$\mathbf{X}$

$\mathbf{y}$

$\alpha$

$\beta$

$\eta$

$\pi_{\mathrm{c}}$

p

$\tau$

$\omega$

$\underline{\text { Subscripts }}$

a

ad

i

$\mathbf{j}$

m

$N$

pc

$\mathbf{r}$

S_tot

$\mathbf{u}$
Axial axis for CompFlow

Tangential axis for CompFlow

Absolute flow angle

Relative flow angle

Efficiency

Overall total pressure ratio for the compressor

Density

Overall total temperature ratio for the compressor

Rotational speed of the shaft

Axial component

Adiabatic

Annulus position

Axial station

Mean or average value

Last annulus position (blade tip)

Polytropic

Radial component

Last axial station

Tangential component 


\section{Chapter 1}

\section{Introduction}

\subsection{The Basics of Jet Engines}

A jet engine can be viewed as a collection of various complicated subsystems. In order to determine the overall efficiency and mechanical stability of a particular engine, each of the major subsystems will need to be analyzed. In general, a jet engine is considered to be a type of turbomachinery, which is a machine where the energy of a moving fluid is changed through the use of moving blades [1]. Rotordynamics is the study of rotating machinery and the analysis of "mechanical side effects" due to operation that lead to vibrations [2]. Vibrations can exist in three primary modes: lateral, torsional, and axial. Also, the interaction of fluid on the rotors and stators produce forces that can lead to self-excited vibrations, which when sustained can lead to stability issues [2]. The field of rotordynamics focuses on predicting machinery responses to different operation modes and the corresponding vibrations.

A jet engine has three major components associated with it downstream of the fan blades: the compressor, combustor, and turbine. After the moving fluid passes through the fan blades it encounters the compressor, which consists of alternating rows of rotating (rotors) and stationary (stators) blades. To prepare the air for combustion, the overall pressure is increased as it passes through the compressor. The combustor injects and mixes fuel into the air, then ignites the mixture for burning. The hot combustion products 
then pass through the turbine, where energy is extracted to drive the compressor and fan. The turbine also consists of alternating rows of rotors and stators. The work of this thesis will focus on analyzing jet engine compressors.

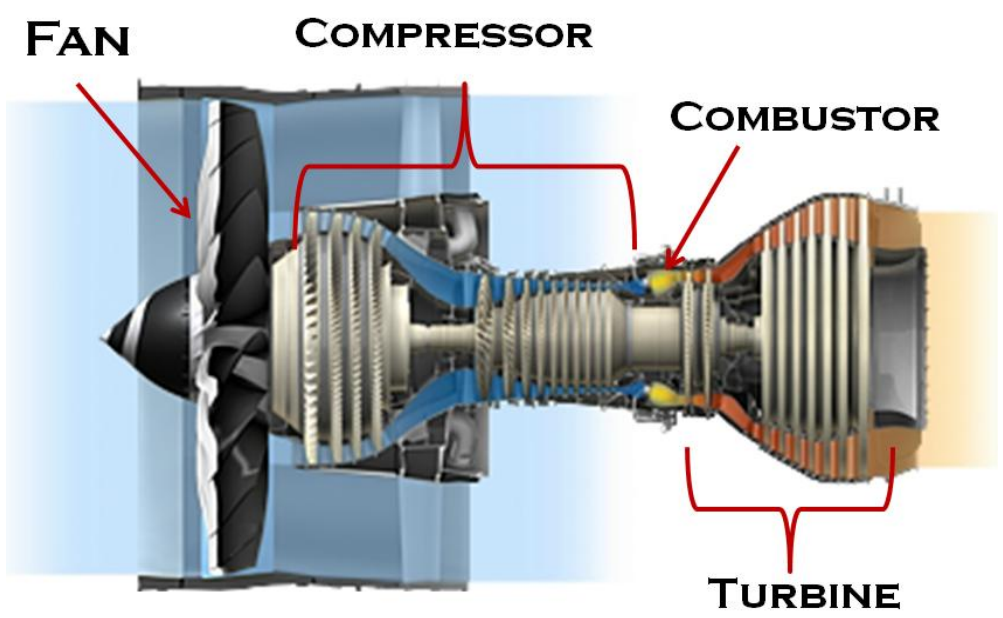

Figure 1.1: Schematic showing main components of high-bypass ratio turbofan [3]

\subsection{Overview of Compressors}

For turbomachinery, there exist three types of compressors: axial, radial, and mixed. Each type is classified with respect to the flow path. For axial flow, the flow is primarily in the axial direction, and for radial compressors the flow is mostly radial [4]. Mixed-flow turbomachinery has a combination of radial and axial flow. As the majority of modern jet engines employ axial flow compressors, this thesis will focus on that category. The focus of this thesis is narrowed to axial-flow compressors, as the compressor is the first type of turbomachinery the air encounters in a jet engine. 
The compressor is an important component within a jet engine because the performance of the compressor will affect the performance of the combustor and turbine as well. As mentioned before, the compressor adds kinetic energy to the moving fluid through the use of rotating blades, which leads to an increase in the total pressure [5]. A rise in the static pressure is achieved by decelerating the flow through the rotor and stator blades of the compressor [6]. A compressor is organized by stages, where each stage contains a rotor and a stator. Additionally, the first stage in a compressor may also contain an inlet guide vane (IGV), which directs the fluid from the fan into the compressor. Most modern jet engines have two sets of compressors that rotate at different rotational speeds on coannular shafts. The collection of rotating-stationary blade pairs rotating at a lower speed make up the low-pressure compressor (LPC), and the pairs that are located further downstream and rotating at a higher speed are labeled the highpressure compressor (HPC).

\subsection{Fluid Dynamics of Compressors}

Aerodynamic forces within an axial compressor could lead to self-excited vibrations, which could lead to instability and mechanical failure [7]. Knowing the force loading on the blades in the compressors would therefore be a necessary parameter for rotordynamics calculations. However, in order to determine the extent of this force, it is necessary to analyze the flow of the fluid through the compressor first. In the case of a jet engine, the analysis of the fluid flow is crucial in order to determine any points of instability and to prevent failure during operation. Therefore, the primary goal of this 
thesis is to determine the aerodynamic forces acting upon the blades of axial compressors for further use in rotor dynamic calculations.

The analysis increases in complexity once the three dimensionality of the fluid is included. The use of a full Navier-Stokes solver is avoided in this case to prevent a computationally expensive procedure. Instead, a "through-flow" analysis is adopted, in which various assumptions are used to simplify the equations of motion. Dixon describes the three common variations of the through-flow method: streamline curvature, matrix through-flow or finite-difference solutions, and time-marching [1]. The streamline curvature method is the oldest method and requires an iterative process. The matrix through-flow method uses radial equilibrium to calculate fluid elements at axial locations along a blade row. The last method is the slowest and requires a large number of iterations to converge. The next section summarizes the literature research conducted on various computational fluid dynamic methods.

\subsection{Project Overview}

The overall goal of this thesis is to determine the loading on compressor blades due to aerodynamic forces. Many fluid dynamic solvers used in industry involve the approximate solution of the Navier-Stokes equations, using procedures generally called computational fluid dynamics (CFD). Use of CFD greatly increases the computational power and time needed for a case. Even with the use of parallel processing (meaning the CFD calculations are spread over multiple computers) the computational time could last days depending on the complexity of the geometry. Therefore, a two-dimensional or 
quasi-three-dimensional approach will be utilized. After research, a "through-flow" method was selected because it provides a good compromise between computational speed and the amount of empirical data necessary for the calculations. As stated earlier, the scope of this thesis for jet engine analysis will be narrowed to axial compressors.

The Rotating Machinery (ROMAC) research group at the University of Virginia has been developing a software package that will allow for the analysis of different types of turbomachinery. The majority of the modules included in this package deal with rotor dynamics and stability calculations. The efforts of this thesis will create an "aerodynamic module" that will added to the software package. Since the main computational programs within the ROMAC software suite have been written most recently in Matlab, this commercial package has also been chosen for ease of integration. The uniqueness of this project is derived from the fact that the streamline method has been directed for rotordynamics calculations and specifically for blade force calculations. The codes written for the aerodynamic module will be generally referred to as CompFlow in the remainder of the thesis.

\subsection{Organization of Thesis}

Chapter 2 discusses the development of various mathematical methods for axial compressors and turbomachinery fluid dynamics. It further expands on the selection of the through-flow method as the ideal tool for the flow calculations. Chapter 3 presents the methodology and governing equations used within CompFlow. Chapter 4 discusses the implementation of CompFlow on several validation cases and presents the results of the 
agreement with experimental data. Chapter 5 presents a full aerodynamic analysis using the GEnx-2B low-pressure compressor and high-pressure compressor as a case study. For this final test, the results will be coupled by using the exit conditions of the low-pressure compressor as the inlet conditions for the high-pressure compressor. 


\section{Chapter 2}

\section{Literature Research}

\subsection{Overview of Mathematical Models}

The flow within most turbomachinery itself is very complex. This section will provide a summary of the major mathematical models used to analyze flow through turbomachinery. The figure below represents the relationship between the various models. At the far right denoted by blue is the one-dimensional model, also referred to as a mean-line model. The middle region represents the two-dimensional models, known as streamline. The far left represents the fully three-dimensional codes, generally known as Navier-Stokes models or computational fluid dynamics (CFD). Each of these types of models is further discussed below.

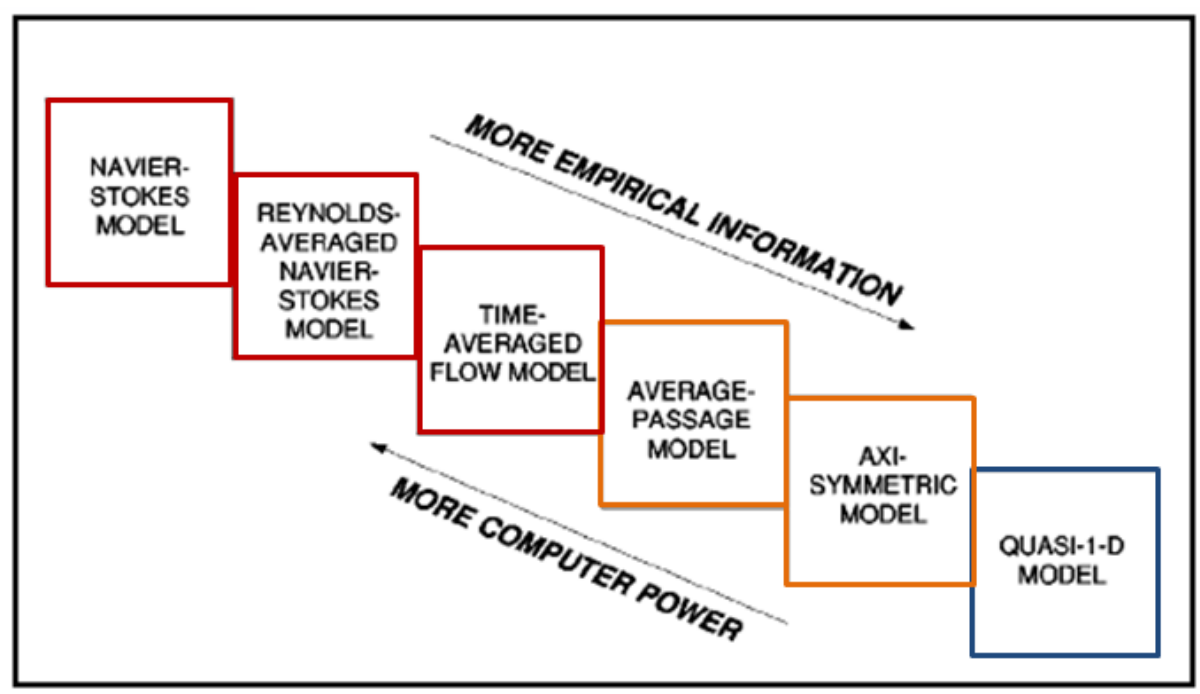

Figure 2.1: Range of Mathematical Models. [8] 


\subsubsection{Mean-line Models}

As seen in the figure above, mean-line models are the least computationally expensive, but require the most amount of empirical information. Since mean-line methods are computationally cheap, they are useful for preliminary design phases where rapid changes are being made to the turbomachinery design. However, these methods are not sufficient for detailed and complex designs [8]. The mean-line method can be considered a simplification of the two-dimensional through-flow analysis discussed next, where variations from hub-to-shroud are ignored [9]. However, as computers have become more powerful, the mean-line method has increasingly been replaced with higher-order methods.

Veres presents a mean-line method that was used for both axial and centrifugal compressors [10]. This mean-line code was written in order to fulfill a need for an efficient method during the conceptual design phase of single or multistage compressors. The thermodynamic and fluid calculations are computed at the mean radial location, as seen below in Figure 2.2. The equations used by the code presented by Veres are based on compressible fluid flow, and the code was validated using various compressor stages. These cases included the single stage axial compressor NASA 37, and the three stage axial compressors NASA 74-A and NASA 76-B. The same data from these mean-line cases reported by Veres are used in to compare the results from CompFlow. These results will be discussed in later chapters. 


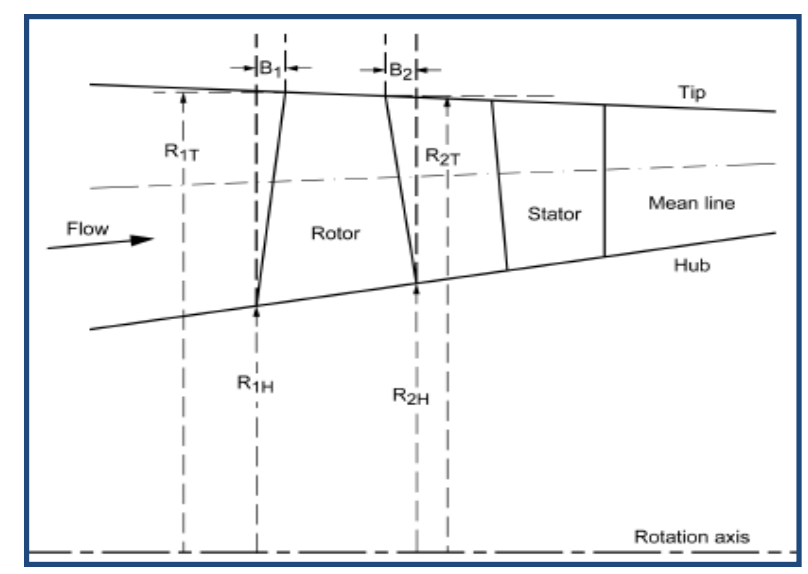

Figure 2.2: Example of mean-line geometry for a single stage [10]

\subsubsection{Streamline Models}

The streamline model is still one of the most widely used methods in industry. To date, it still represents a good compromise between computational expense and extensive knowledge of geometry. In 1952, Wu was the first to reduce the three-dimensional flow through turbomachinery into two intersecting "stream surfaces", which he refers to as S1 and S2 [11]. The through-flow analysis deals with solving equations in the hub-to-shroud plane at stations in between blade rows. In a stream surface, there is no fluid velocity component normal to it and mass does not flow across it [9]. One of the major simplifications for the through-flow method is that flow is assumed to be axisymmetric. This allows for calculations to be done for a single representative blade within an entire row.

The streamline method was deemed to be the most favorable within the project scope. Therefore, a later section will detail the development of the streamline method. 
Chapter 3 will discuss how the streamline method is implemented within the code. The figure below is a graphical representation of the two types of stream surfaces.
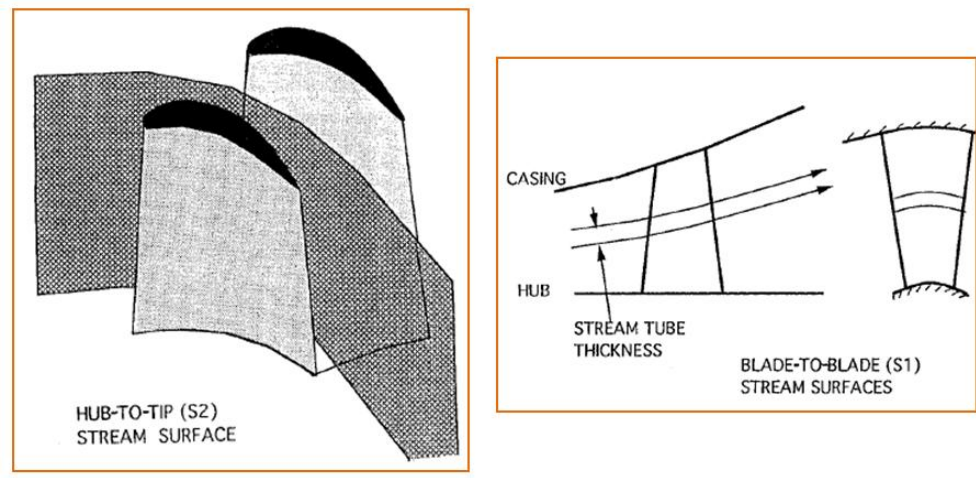

Figure 2.3: S1 (right) and S2 (left) stream surfaces [12]

\subsubsection{CFD}

In 1952, Wu was one of the first to recognize that flow in turbomachinery is three-dimensional in nature, but during his time the computational power necessary for the full 3D calculations were not available [13]. Indeed, use of fully 3D models did not become prevalent in industry until the 1990s. There are various mathematical reductions that can be used for applying 3D models, but the most common are time-marching solutions for the Euler (inviscid) or Navier-Stokes equations (viscous) [12]. The primary purpose of using these 3D methods is to predict secondary flows.

Calculations can be done for a single blade row or multistage geometry. However, the required computational time and power is extensive for the increasing degree of geometric complexity. For example, for a mesh containing 70,000 grid points per blade row and having twelve blade rows, such a solution might take 24 hours on a modern workstation [12]. One major issue is that CFD is still not the main part of the design 
phase, being used primarily for checking a particular design as a post-analysis step [14]. A reason for this can be attributed to the fact that CFD is not agile enough to account for the rapid changes to the geometry during the preliminary design phase. This method also often requires manual manipulation of meshes before a case can be run, which is a timeconsuming process. An example of a 2D mesh around a single blade is shown below in Figure 2.4.

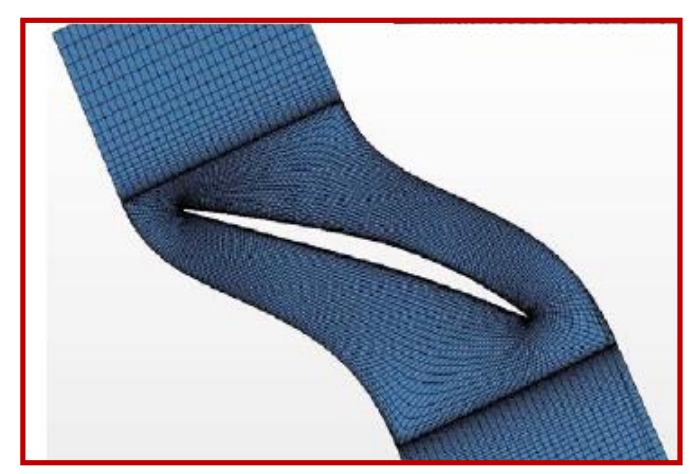

Figure 2.4: CFD mesh of a single blade [15]

\subsection{Development of Streamline Model}

Wu's sophisticated approach to breaking the three-dimensionality of turbomachinery flow into two intersecting stream surfaces is shown below in Figure 2.5. The S1 surface refers to the "through-flow" or "meridional flow", as shown in red. The flow in between blades, from hub to tip as outlined in blue, is referred to as the S2 stream surface. In order to obtain a good prediction of the three-dimensionality of the flow, the two stream surfaces methods must be coupled [14]. For example, the blade flow angles determined using the $\mathrm{S} 2$ method are the input for the $\mathrm{S} 1$ method in order to predict the streamlines. 


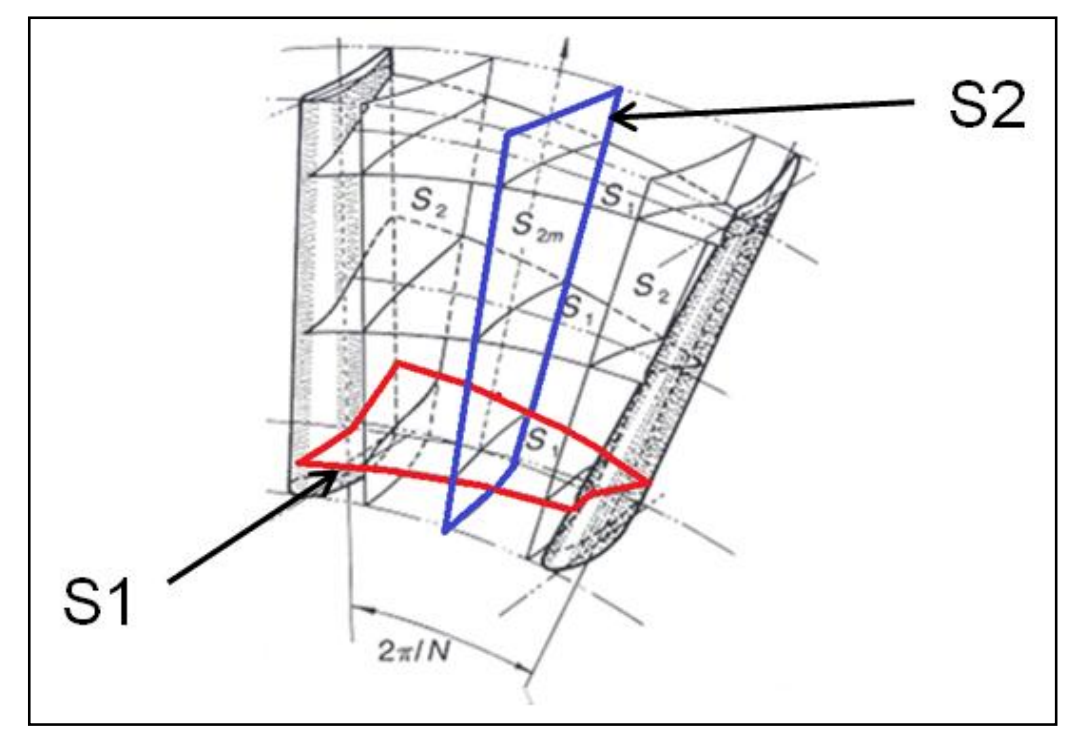

Figure 2.5: Intersection of S1 and S2 stream surfaces [16]

Wu's theory was not implemented until 1966 when Marsh presented a computational method using matrices. The through-flow method was used to get the overall flow pattern while ignoring viscosity or time-dependent flow characteristics. The equations were solved assuming steady, axisymmetric inviscid flow [17]. Dixon also provides a summary of through-flow methods which include streamline curvature, matrix throughflow or finite-difference solutions, and time-marching solutions [1].

As recently as 1998, Denton and Dawes refer to the through-flow method as the "backbone of turbomachinery design" [12]. The S1 calculations can be used to determine the shape of various turbomachinery blades, and the through-flow calculations determine the span-wise changes in flow angle at the inlet and exit of each blade row. Chapter 3 will discuss the implementation of the through-flow method in CompFlow. 


\section{Chapter 3}

\section{CompFlow Methodology}

\subsection{Introduction to CompFlow}

The equations governing CompFlow were developed primarily using the derivations of Flack [5]. This chapter will discuss the assumptions used to reduce the equations of motion into algebraic expressions. Flack uses a control volume approach by dividing the flow through a compressor into a finite number of annuli. Axial stations are chosen to be located in front of and behind each blade row. The flow is then analyzed at each axial station for each annulus, moving from hub to casing and marching downstream axially. A schematic is shown below in Figure 3.1, where axial stations are denoted by $j$ and annuli by $i$. The streamlines, denoted by dashed lines, shown below represent the S2 stream-surface, flow designated from hub to tip, as denoted by $\mathrm{Wu}$ and shown earlier in Figure 2.5.

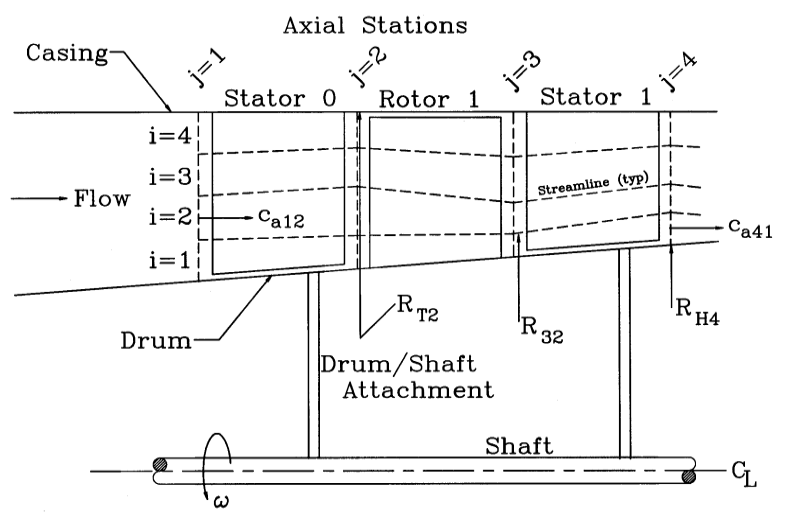

Figure 3.1: Depiction of annulus and axial station positions 


\subsection{Assumptions}

The flow through an actual compressor will exhibit three-dimensionality as well as viscous and unsteady operating conditions. The real flow field is very complex, with boundary layers and areas of laminar and turbulent flow and secondary flows [18]. Various assumptions were made to reduce the flow field to two-dimensional steady flow, which are discussed further below. The streamline analysis method derived by Flack assumes that the fluid flow around blade rows is axisymmetric, which allows for calculations to be done on a single representative blade for each row.

There are multiple levels within the streamline analysis model, and the one employed by Flack is derived using the simple radial equilibrium theory. This theory is based on the idea that the all radial flow in a blade passage occurs inside the blade row, with flow directly outside the blade row in radial equilibrium [1]. The flow is also assumed to be steady throughout the blade passage, where transient responses are not considered and the blade rows are analyzed at a "frozen" position. Adiabatic flow is also assumed, meaning there is no heat transfer from the fluid [18]. Therefore, the governing equations in CompFlow are based on the combination of conservation of mass, the simple radial equilibrium equation, Euler's turbine equation, and various supporting equations for a perfect gas [18]. The next section will explain the derivation of the radial equilibrium equation. 


\subsubsection{Derivation of Simple Radial Equilibrium Equation}

The application of different assumptions to the radial momentum equation will yield different forms of radial equilibrium. For simple radial equilibrium, variations of the radial velocity are assumed to be negligible. Also, surface curvature is neglected and the variation of entropy inside the blade row is ignored [9]. A model that includes the entropy gradient is referred to as a "simple non-isentropic radial equilibrium" and including both curvature and entropy is called "full radial equilibrium" [9]. The following derivations for CompFlow are based on the principles of "simple radial equilibrium". The governing equation is derived from the radial momentum equation, as shown below [4]:

$$
\frac{\partial V_{r}}{\partial t}+V_{r} \frac{\partial V_{r}}{\partial r}+V_{x} \frac{\partial V_{r}}{\partial x}-\frac{V_{u}^{2}}{r}=-\frac{1}{\rho} \frac{\partial p}{\partial r}+F_{r}
$$

Looking at Equation (3.1) shown above, the radial direction is denoted by the subscript $r$, the axial direction by $x$, and the tangential direction by $u$. For steady flow, variations with respect to time are set to zero, which means the first term on the left side of Equation (3.1) disappears. As the radial velocities are negligible, the variations in the radial and axial direction are very small [16]. Therefore, the second and third term on the left side can also be dropped. Cumpsty also states that for most axial compressors the radial force due to the blade $\left(F_{r}\right)$ on the fluid is small enough to drop [16]. Therefore, the radial equilibrium equation is reduced to the form shown below.

$$
\frac{\partial p}{\partial r}=\rho \frac{V_{u}^{2}}{r}
$$


Flack arrives at the same conclusion using differential analysis on a radial wedge of fluid [5]. Figure 3.2 displays that the wedge sweeps an angle of $d \theta$, and extends from a radius of $r$ to $r+d r$. The pressure acting on the inner radius of the wedge is $p$, and on the outer radius $p+d p$. The pressures on the sides are equal to $p+d p / 2$. The summation of the pressure forces must equal the centrifugal forces for radial equilibrium. As the tangential forces cancel out, only the radial components are shown below [5]:

$$
(p+d p)(r+d r) d \theta-p \cdot r \cdot d \theta-2\left(p+\frac{d p}{2}\right) d r \frac{d \theta}{2}=\frac{d m}{2} c_{u}{ }^{2}
$$

The differential mass is given by:

$$
d m=\rho \cdot r \cdot d \theta \cdot d r
$$

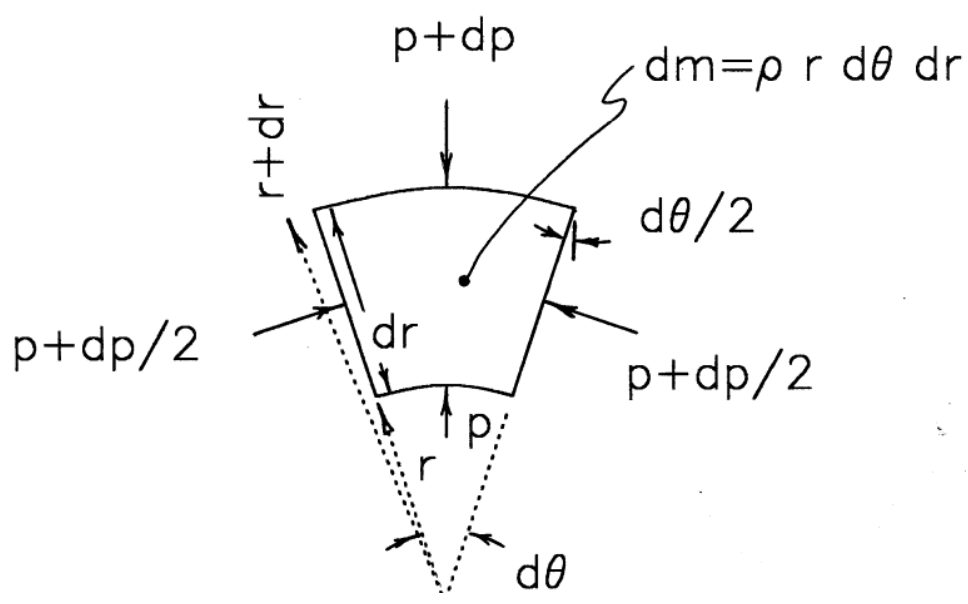

Figure 3.2: Wedge of radial fluid for radial equilibrium derivation [5]

In Flack's derivations, the axial direction is denoted by $a$, and the tangential direction by the subscript $u$. Using Equation (3.3) and neglecting higher order terms, the results match up with Equation (3.2). This result shows that the radial pressure gradient is a function of tangential velocity and radius. By applying different constraints, various 
tangential velocity distributions can be reached that satisfy radial equilibrium. For example, Equation (3.5) shows the "free vortex" distribution in which the tangential velocity varies inversely with the radius, which will be discussed later. Other distributions that also satisfy radial equilibrium are shown below, where Equation (3.6) represents "forced vortex" and Equation (3.7) represents "half-vortex" [19].

$$
\begin{gathered}
c_{u}=\frac{a}{r} \\
c_{u}=b r \\
c_{u}=\frac{a}{r}+b r
\end{gathered}
$$

Early efforts for this thesis showed that the code calculations would yield axial velocities that increased significantly across a compressor, leading to proportionally high Mach numbers. This intuitively did not make sense as the main purpose of the compressor is to slow the flow down at intervals in order to achieve a pressure rise. The component trends for an axial compressor that were sought in the calculations are summarized by Flack and shown in the table below [5].

Table 3.1: Trends for components in axial compressors

\begin{tabular}{|l|c|c|c|c|c|}
\hline & $\begin{array}{c}\text { Absolute } \\
\text { Velocity }\end{array}$ & $\begin{array}{c}\text { Relative } \\
\text { Velocity }\end{array}$ & Area & $\begin{array}{c}\text { Static } \\
\text { Pressure }\end{array}$ & $\begin{array}{c}\text { Total } \\
\text { Pressure }\end{array}$ \\
\hline IGV & increases & increases & decreases & decreases & constant \\
\hline Rotor & increases & decreases & increases & increases & increases \\
\hline Stator & decreases & decreases & increases & increases & constant \\
\hline
\end{tabular}

The prior research yielded results that showed for a simple compressor case, the calculations done using the radial equilibrium equation shown in Equation (3.2) matched with the given data, even following the velocity trends in the table above. However, a compressor case with a more complex geometry, such as a narrowing duct, led to 
increasingly higher values of axial velocity. These results could be attributed to the neglect of significant curvature in the geometry by the radial equilibrium expression. To account for this, Equation (3.2) was modified to account for the axial velocity gradient seen in Equation (3.1). This modification was successful in preventing the axial velocity from increasing drastically across the compressor.

The derivation for the modified equation begins with Equation (3.2) and using the thermodynamic expression for entropy as below as described by Lakshminarayana [4]:

$$
\frac{1}{\rho} \frac{d p}{d r}=\frac{d h}{d r}-T \frac{d s}{d r}
$$

The static enthalpy is then substituted by the stagnation enthalpy yielding:

$$
h_{0}=h+\frac{V^{2}}{2}=h+\frac{V_{u}^{2}}{2}+\frac{V_{x}^{2}}{2}
$$

Finally, Equation (3.9) is substituted into the right hand side of Equation (3.8), which with some rearranging yields:

$$
\frac{\partial p}{\partial r}=\rho\left(\frac{V_{u}{ }^{2}}{r}+V_{u} \frac{\partial V_{u}}{\partial r}+V_{x} \frac{\partial V_{x}}{\partial r}\right)
$$

The above equation was applied in the direct mode of calculations using a discrete form. More information on this implementation is provided in Section 3.3.2 starting on page 30 . 


\subsubsection{Viscosity Effects}

Although the real flow through the compressor blade passage is viscous in nature, inviscid flow is primarily assumed for ease of calculations. This condition means that any terms associated with transport phenomena are neglected, including viscosity $(\mu)$, mass diffusion and thermal conductivity [20]. Even though viscous effects are not fully considered in this code, a discussion of these effects is done to provide a background for other computational models. Viscosity will introduce losses in the compressor, and the preliminary design phase should be used to limit the amount of loss [18]. In CompFlow, loss is included within the efficiency distributions behind each blade row, which is entered as an input parameter. The efficiency entered into CompFlow is the adiabatic efficiency, which is the ratio of the isentropic work to the actual work of a compressor blade. The area along a blade where viscosity has the largest effect is called the boundary layer, as shown in Figure 3.3 [16]. Since a compressor is inherently an environment of adverse pressure gradients, boundary layers may be thick and have a tendency to separate, which is compounded by secondary flows and tip leakage [19].

Cumpsty states that viscosity has three major effects on compressor performance: it puts a limit on the pressure rise, it leads to flow blockage, and it leads to loss generation [16]. Excessive pressure rise in the compressor could lead to flow instability, surge, and rotating stall. Blockage occurs when the flow area is effectively reduced due to boundary layer interactions, which will impact the mass flow through the compressor and the work required. Cumpsty also asserts that blockage is of larger importance to designers than loss generation, even though loss generation is important for determining the maximum pressure rise in the compressor [16]. 
Modeling these viscous effects can be difficult, and though experimental values of loss yield good matching, empirical correlations may be restricted to a small range of different types of compressors [12]. Denton and Dawes do refer to a viscous throughflow method written by Gallimore as one of the most sophisticated in that category, as it models the entropy at the endwalls as well as losses due to secondary flows and tip leakage [12]. Conventional boundary layer theory is not applicable for endwall calculations as the flow is inherently three-dimensional in character [19]. In general, as the viscous effects are best calculated using the blade-to-blade (S1) stream surfaces, the assumption of inviscid flow in the meridional (S2) surface is valid.

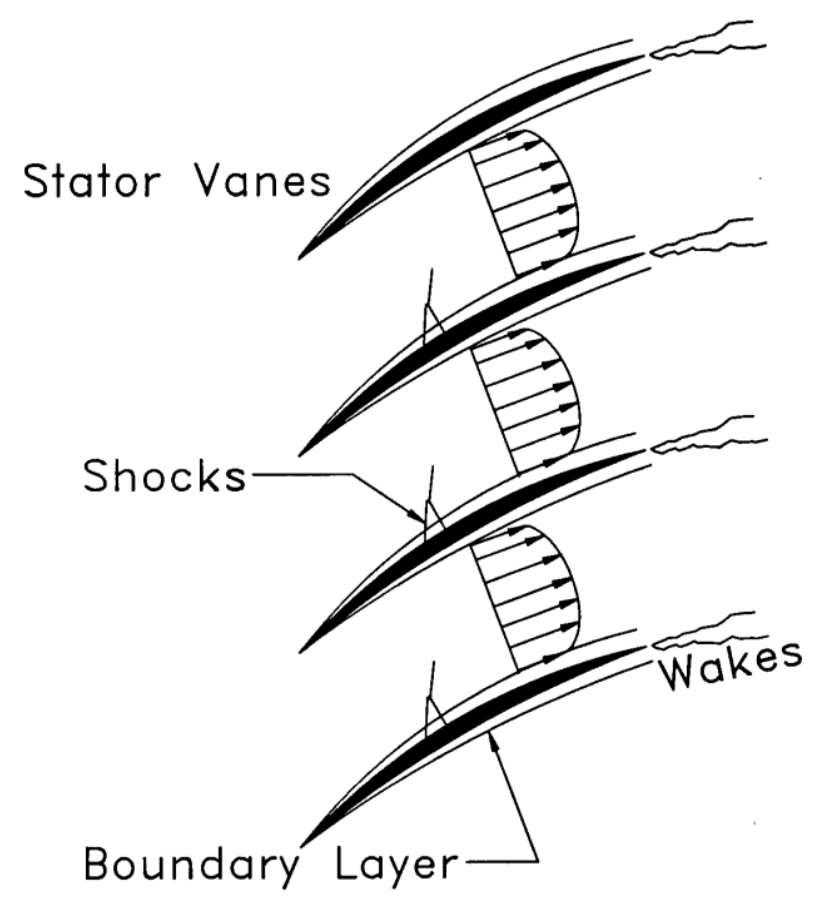

Figure 3.3: Boundary layers and wakes along the surface of stator blades [5] 


\subsubsection{Compressibility Effects}

In his derivations, Flack does not account for changes in density across a compressor. Instead, his streamline analysis method assumes incompressible flow. However, real flow through a compressor does not have a constant density and there may be regions of supersonic flow along the rotor and stator blades. Such a compressor is referred to as a transonic compressor, where the inlet Mach number varies from 0.4 to 0.7 [18]. At this time, CompFlow has been developed to address transonic compressor cases only. In general, a flow cannot be considered incompressible if the Mach number is greater than 0.3 , which is usually the case for most axial compressors. Therefore, compressibility has been included in the equations of CompFlow. The density will increase as the air moves axially downstream through the compressor, which will lead to a decrease in axial velocity [4].

The calculations described in this thesis will employ a "pseudo-compressible" model, where the fluid is assumed to be incompressible within a stage, with density changes occurring in between stages. As the temperature rise across a compressor stage is small, the corresponding density change will be small as well. Therefore, a mean density can be applied within a stage [1]. A fully compressible model was not utilized at this time in order to simplify the governing equations and reduce computational run-time. The density increase will be calculated at the exit of each stage as the inlet condition for the next using the ideal equation of state [1]:

$$
p=\rho R T
$$




\subsection{Governing Equations}

The streamline analysis model can be applied in various ways by specifying different input parameters. Compflow will offer two modes of calculation: indirect, and direct. The equations for the different modes are based on velocity polygons, as shown in Figure 3.4. The indirect calculation mode couples the condition of free vortex with radial equilibrium, meaning the tangential velocity varies inversely with the radius [21]. The direct mode requires iterations for the calculation of the streamlines for specified flow angle distributions. If the distribution of total pressures and total temperatures is known throughout the compressor, the design mode of calculations can be used. The equations associated with each mode are expanded upon in the Sections 3.3.1 and 3.3.2.

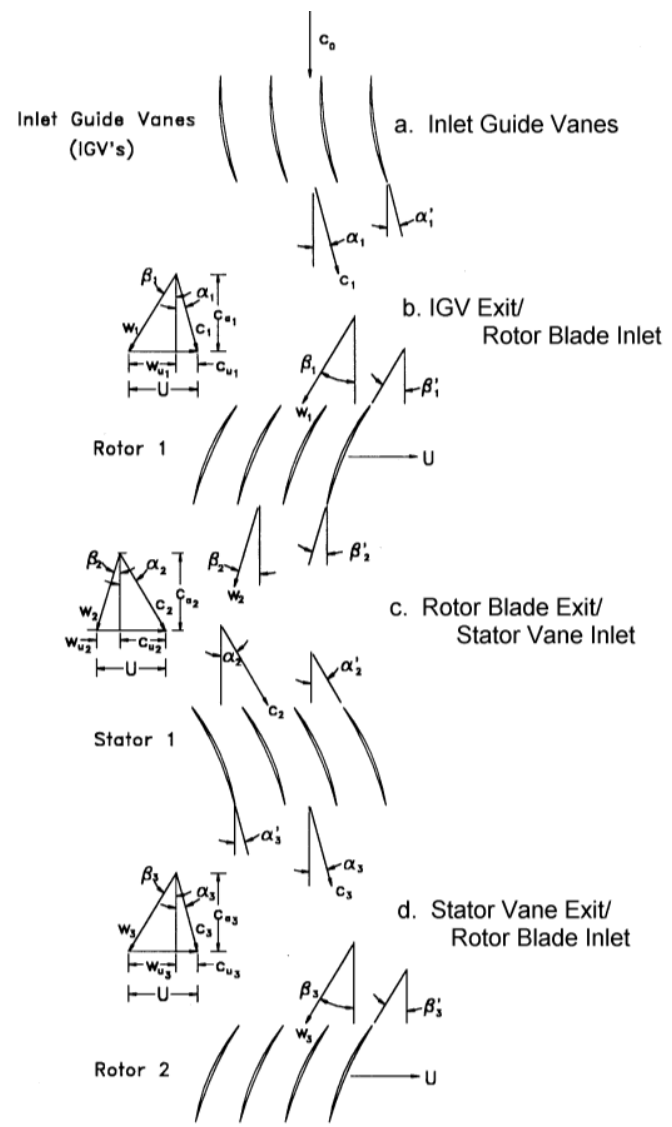

Figure 3.4: Velocity polygons for axial compressors [5] 


\subsubsection{Indirect Mode}

As stated earlier, the indirect mode is based on a reduction of the simple radial equilibrium expression shown in Equation (3.2). The radial equilibrium expression is coupled with the free vortex condition, which is derived by simplifying the radial equilibrium expression in Equation (3.10). Korpela reduces the simple non-isentropic radial expression to Equation (3.12) below by assuming steady flow with no pressure gradients or body forces in the radial direction [21]:

$$
V_{x} \frac{d V_{x}}{d r}+V_{u} \frac{d V_{u}}{d r}+\frac{V_{u}^{2}}{r}=0
$$

If the axial velocity is assumed to be constant through the compressor, then rearranging the equation will yield the expression below in Equation (3.13), for which the solution is that $r V_{u}=$ constant.

$$
\frac{d V_{u}}{V_{u}}=-\frac{d r}{r}
$$

Flack provides a discrete version of this solution using the average radius, which is applied by the code and shown here [5]:

$$
c_{u_{i j}} \bar{R}_{i j}=c_{u_{i+1 j}} \bar{R}_{i+1 j}
$$

The subscript $i$ refers to the annular position and the subscript $j$ refers to the axial station. As seen below in Equation (3.15), any quantity with a bar over it, such as $\bar{R}_{i j}$, represents the average value across two annuli or axial stations. Axial and tangential directions are specified by the subscripts $a$ and $u$ respectively. For instance, 


$$
\bar{R}_{i j}=\frac{1}{2}\left(R_{i j}+R_{i-1 j}\right)
$$

The input parameters for the indirect mode of calculations are summarized in the table below. The inlet parameters can be initialized by specifying either static pressure or total pressure and total temperature. Note that several of the parameters, such as efficiency $(\eta)$, require radial distributions for each axial station (blade row). The total number of axial stations is calculated by multiplying the number of stages by two (accounting for a rotor/stator per stage) accounting for the IGV and adding another station for ambient conditions, which are used for initialization.

Table 3.2: Input parameters for indirect mode

\begin{tabular}{|c|c|}
\hline Number of annuli & $n$ \\
\hline Number of stages & $S$ \\
\hline Is there an inlet guide vane? $(1=$ yes, $0=$ no $)$ & $I G V$ \\
\hline Total number of axial stations & $S_{\text {tot }}$ \\
\hline \multicolumn{2}{|l|}{ Names of stations } \\
\hline \multicolumn{2}{|l|}{ Units (1 = English, $2=$ Metric) } \\
\hline Rotational speed (rpm) & $\omega$ \\
\hline Hub radius for each station & $R_{h}$ \\
\hline Tip radius for each station & $R_{t}$ \\
\hline $\begin{array}{l}\text { Static pressure OR Total pressure and total } \\
\text { temperature }\end{array}$ & $p, P_{t}, T_{t}$ \\
\hline Inlet density & $\rho$ \\
\hline Mass flow rate & $\dot{m}$ \\
\hline $\begin{array}{l}\text { Exit absolute flow angle distribution for each } \\
\text { blade row }\end{array}$ & $\alpha$ \\
\hline Efficiency distribution for each stage & $\eta$ \\
\hline
\end{tabular}

The indirect method begins the calculations by equally spacing annuli in the radial direction through the compressor. Next the inlet axial velocity, denoted by the subscript $a$, will need to be estimated either by the code using Equation (3.16), where $\dot{m}$ is the mass flow rate, or through manual user input. 


$$
c_{a}=\frac{\dot{m}}{\rho A}
$$

Equation (3.17) shows the calculation for the inlet flow area.

$$
A=\pi\left({R_{T}}^{2}-R_{H}{ }^{2}\right)
$$

Although the free vortex condition calls for a constant axial velocity and density (incompressibility) throughout the compressor, CompFlow will apply a "pseudocompressible" model. Literature research and empirical data shows that although the axial velocity is primarily constant throughout the compressor, there is a slight decrease in the value as the flow proceeds downstream. This trend will be enforced in CompFlow by decreasing the axial velocity at each axial station by a small percentage $(\sim 1 \%)$ compared to the previous station. The user will have the ability to enter the percentage values in the GUI. This will force the density to vary across the compressor, and therefore apply a compressibility condition.

For this mode of calculation, the flow angles at the exit of each station need to be specified for mid-stream values only. Mid-stream refers to the annulus located half-way between the hub and tip radius for an axial station, which is essentially the mean radius. The mid-stream values for the tangential and total velocities are determined by using the specified flow angles and the midstream values of the compressor axial velocity. This is accomplished by using the following three equations:

$$
\begin{gathered}
c_{a_{i j}}=c_{i j} \cos \alpha_{i j} \\
c_{u_{i j}}=c_{i j} \sin \alpha_{i j} \\
c_{i j}{ }^{2}=c_{a_{i j}}{ }^{2}+c_{u_{i j}}{ }^{2}
\end{gathered}
$$


Values of the axial $\left(c_{a}\right)$ and tangential $\left(c_{u}\right)$ velocities at the remaining radial positions are obtained by using the free vortex equation derived earlier in Equation (3.14).

Conservation of mass states that the amount of mass entering the control volume is equal to the amount leaving. Since the annuli represent control volumes, no mass flow will cross the streamlines. Flow will enter and leave only at the axial faces [5]. Therefore the volumetric flow rate is calculated by evenly distributing the mass flow rate over the annuli and dividing by the density, as seen in Equation (3.21). The top of the annulus, denoted by $R_{i+1 j}$, is determined by using Equation (3.22).

$$
\begin{gathered}
Q_{j}=\frac{\dot{m}}{\rho_{j} n} \\
Q_{j}=c_{a_{i j}} \pi\left({R_{i+1 j}}^{2}-{R_{i j}}^{2}\right)
\end{gathered}
$$

Since the mass flow rate is evenly divided across the annuli, and the density is assumed to be constant from hub to tip at an axial station, the volumetric flow rate will be constant for an axial station. Other parameters calculated include the blade velocity $(U)$, seen in Equation (3.23), where $\omega$ is the rotational speed in radians per second.

$$
U_{i j}=\bar{R}_{i j} \omega
$$

The torque $\left(T_{q}\right)$ on the shaft due to a particular annulus is given by the Euler turbine equation:

$$
T_{q_{i j}}=Q_{j}\left(\rho_{j+1} \bar{R}_{i j+1} c_{u_{i j+1}}-\rho_{j} \bar{R}_{i j} c_{u_{i j}}\right)
$$

The power $(\dot{W})$ added to each annulus is given by Equation (3.25). The rotational speed $(\omega)$ is set to zero when stator blades are being analyzed. 


$$
\dot{W}_{i j}=T_{q_{i j}} \omega
$$

The relative exit flow angle $(\beta)$ can also be calculated using the blade velocity and velocity components:

$$
\tan \beta_{i j}=\frac{c_{u_{i j}}-U_{i j}}{c_{a_{i j}}}
$$

Equation (3.27) shows how the relative flow angle can also be used with the axial velocity to determine the relative velocity at a point in the flow field.

$$
w_{i j}=\frac{c_{a_{i j}}}{\cos \beta_{i j}}
$$

After the initialization process, the static pressure at the inlet will be known. In order to calculate the static pressure at the next axial station along an annulus, the energy equation is used. Flack's simplification of the energy equation in discrete form [5]:

$$
p_{i j+1}=\rho_{j+1}\left[\dot{W}_{i j} \frac{\eta_{i j}}{\dot{m}_{a}}-\frac{1}{2}\left(c_{i j+1}{ }^{2}-c_{i j}{ }^{2}\right)-\frac{p_{i j}}{\rho_{j}}\right]
$$

In the above expression, $\eta$ is the annular efficiency distribution for a stream tube as specified in the input. The term $\dot{m}_{a}$ is the total mass flow rate, $\dot{m}$, divided by the number of annuli ( $n$ ) being analyzed. Using the static pressure from Equation (3.28), the total pressure can also be determined:

$$
p_{t_{i j}}=\frac{\rho_{j}}{2} c_{i j}^{2}+p_{i j}
$$

Using the pressure ratio across the blade row, the total temperature is determined using: 


$$
T_{t_{i j}}=T_{t_{i j-1}}\left\{\frac{1}{\eta_{i j}}\left[\left(\frac{p_{t_{i j}}}{p_{t_{i j-1}}}\right)^{\gamma / \gamma-1}-1\right]+1\right\}
$$

The static temperature in Equation (3.31), the speed of sound described by Equation (3.32), and the Mach number in Equation (3.33) can all be determined consecutively. These three calculations complete the main computational step for the indirect problem.

$$
\begin{gathered}
T_{s_{i j}}=T_{t_{i j}}-\frac{c_{i j}{ }^{2}}{2 c_{p}} \\
a_{i j}=\sqrt{\gamma \cdot R \cdot T_{s_{i j}}} \\
M_{i j}=\frac{c_{i j}}{a_{i j}}
\end{gathered}
$$

\begin{tabular}{|c|c|}
\hline (1) & Ambient conditions provided as input (first axial station). \\
\hline (2) & Evenly space streamlines throughout compressor $(\mathbf{R})$ \\
\hline (3) & $\begin{array}{l}\text { If there is compressibility, set the value of axial velocity throughout the compressor } \\
\text { using percentage values given. Else, set the axial velocity to ambient conditions. }\end{array}$ \\
\hline (4) & $\begin{array}{l}\text { Calculate } c \text { and } c_{u} \text { at midstream values } M=(n+1) / 2 \text { using Equations (3.18) } \\
\text { and (3.19) }\end{array}$ \\
\hline (5) & Use free vortex condition to obtain remaining $c_{u}$ values using Equation (3.14) \\
\hline (6) & Get remaining values of $c$ using Equation (3.20) \\
\hline (7) & Get remaining values of $\alpha$ using Equation (3.18) \\
\hline (8) & Calculate $U, \beta$ using Equations (3.23) and (3.26) \\
\hline (9) & Calculate torque and power using Equations (3.24) and (3.25) \\
\hline (10) & $\begin{array}{l}\text { For } \mathrm{j}=2 \text { to } \mathrm{s}_{\text {tot }} \text {, calculate } p, P_{t}, T, \text { and } T_{t} \text { using Equations (3.28), (3.29), (3.30), and } \\
\text { (3.31). If the axial station is the exit of a stage, update the velocity. }\end{array}$ \\
\hline (11) & Calculate $a$ and $M$ using Equations (3.32) and (3.33) \\
\hline
\end{tabular}

Table 3.3: Flow of Calculations for Indirect Method 
As stated earlier, density is assumed to be constant through a single stage. Therefore, the density is updated at the exit of each stage, and used as input for the next stage. The values for specific heat $\left(c_{p}\right)$ and $\gamma$ are also updated at this time. Further explanation of these values is provided in Appendix A. The steps for the flow of calculations for the indirect method are summarized below. A graphical representation of the flow of calculations is also shown below.

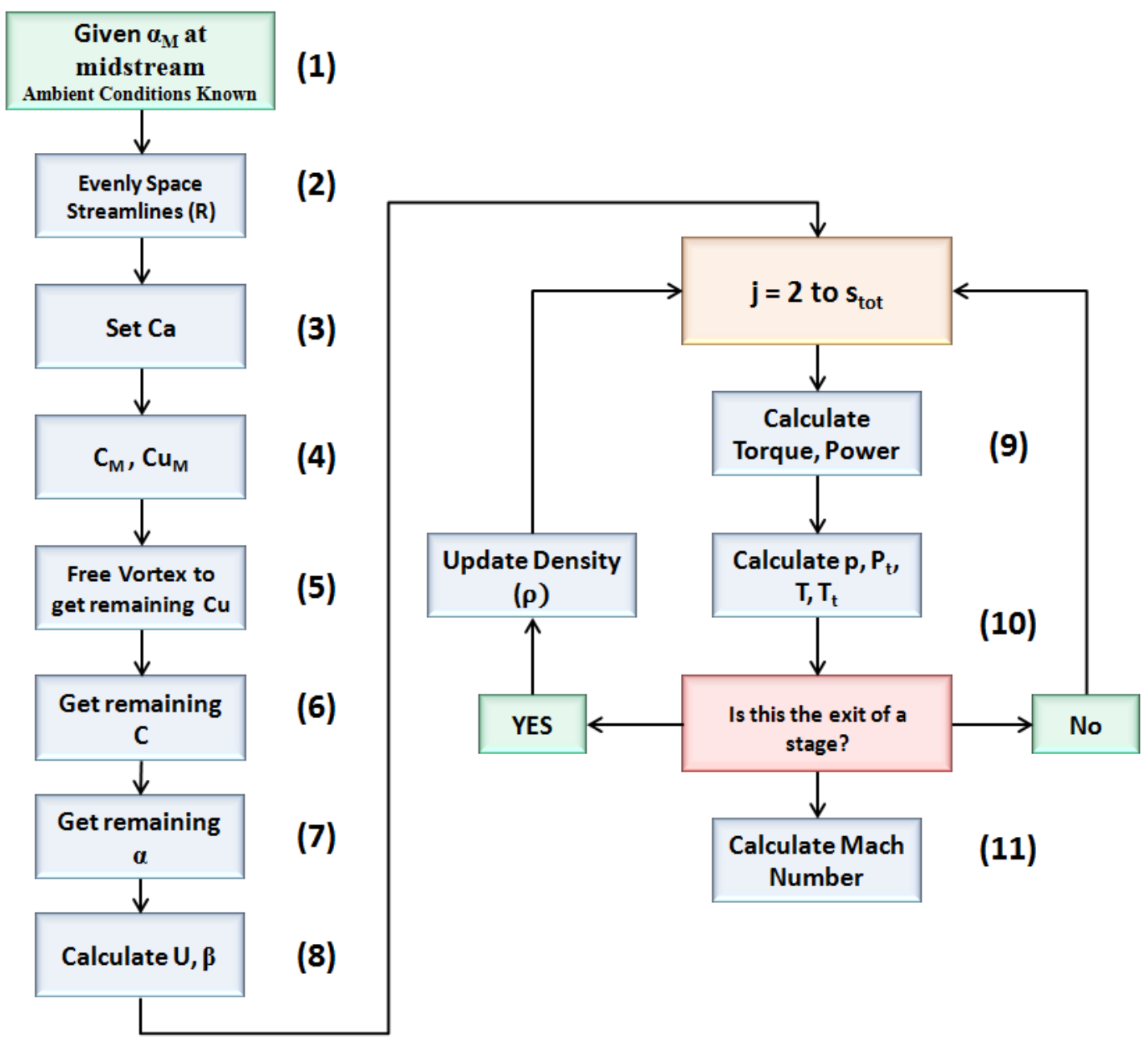

Figure 3.5: Flowchart for indirect mode calculations 


\subsubsection{Direct Mode (Flow Angles specified)}

For the direct mode of calculations, the radial distribution of the exit flow angles and efficiencies is specified for each blade row and the velocity components are computed [1]. Radial equilibrium is assumed at each of the axial stations by using a rearward difference approximation of Equation (3.10), as shown here:

$$
\frac{\left(p_{i j}-p_{i-1 j}\right)}{\bar{R}_{i j}-\bar{R}_{i-1 j}}=\rho_{j}\left[\frac{\bar{c}_{u_{i j}}^{2}}{R_{i j}}+\bar{c}_{u_{i j}} \frac{\left(c_{u_{i j}}-c_{u_{i-1 j}}\right)}{\bar{R}_{i j}-\bar{R}_{i-1 j}}+\bar{c}_{a_{i j}} \frac{\left(c_{a_{i j}}-c_{a_{i-1 j}}\right)}{\bar{R}_{i j}-\bar{R}_{i-1 j}}\right]
$$

This method will require iteration to solve for the velocity components. Also, the size of the stream tubes is found as part of the solution. Flack presents an approach for the direct method of calculations, which is shown in the table below [5].

As used with the indirect mode, the subscript $i$ refers to the annular position, with the value of 1 referring to the hub and $N$ to the tip. The subscript $j$ refers to the axial station, with values increasing as the stations move downstream. The process below involves a double iterative loop in order to carry out the calculations. A schematic of the order of calculations is also shown in Figure 3.6.

As this process involves iteration, a convergence method will need to be used for the axial velocity calculations. These methods seek to find the root of a nonlinear function that describes the physical problem. For the CompFlow calculations, the RegulaFalsi (false position) method is used. The general idea is that two previous values for $x$ and $f(x)$ are used to obtain an estimate of a new $x$. This is done by fitting a linear equation through the two points, with the new value calculated using [5]: 


$$
x_{3}=\left[\frac{x_{1} y_{2}-x_{2} y_{1}}{y_{2}-y_{1}}\right]
$$

Table 3.4: Flow of Calculations for Direct Mode

\begin{tabular}{|c|c|}
\hline (1) & Ambient conditions provided as input (first axial station) \\
\hline (2) & $\begin{array}{l}\text { Increment the axial station }(j=2) \text { and estimate a value for } c_{a_{1 j+1}} \text {, at the hub radius } \\
\text { using Equation }(3.16)\end{array}$ \\
\hline (3) & $\begin{array}{l}\text { Analyze the inner annulus }(i=1) \text { and calculate } R_{i+1 j+1} \text { using mass conservation } \\
\text { and Equation }(3.22)\end{array}$ \\
\hline (4) & $\begin{array}{l}\text { The values for the absolute exit flow angle, } \alpha_{i j+1} \text {, and the efficiency, } \eta_{i j+1} \text {, are } \\
\text { known. }\end{array}$ \\
\hline (5) & Calculate $c_{i j+1}, c_{u_{1 j+1}}, \beta_{i j+1}$. using Equations (3.18), (3.19), and (3.26) \\
\hline (6) & Calculate the torque and power using Equations (3.24) and (3.25) \\
\hline (7) & Calculate the static pressure using Equation (3.28) \\
\hline (8) & Increment to the next annulus $(i=2)$ and repeat Steps 2 through 6 to get $p_{i j+1}$. \\
\hline (9) & Compute static pressure using radial equilibrium and Equation (3.34). \\
\hline (10) & $\begin{array}{l}\text { If the values of static pressure computed in Step } 7 \text { and Step } 8 \text { are equal within a } \\
\text { tolerance, increment } i \text { to analyze the next annulus. If they are not within the } \\
\text { tolerance, estimate a new value of } c_{a_{i j+1}} \text {. }\end{array}$ \\
\hline (11) & $\begin{array}{l}\text { For the last annulus, } c_{a_{N j+1}} \text { can be found from the known value for the tip radius } \\
R_{N j+1} \text {. Steps } 2-8 \text { above are repeated to obtain the static pressure, } p_{N j+1} \text {. }\end{array}$ \\
\hline (12) & $\begin{array}{l}\text { Repeat Step } 9 \text { to obtain the radial equilibrium static pressure at the tip annulus } \\
(i=N) \text {. }\end{array}$ \\
\hline (13) & $\begin{array}{l}\text { If the two values of static pressure computed in Steps } 11 \text { and } 12 \text { are within a set } \\
\text { tolerance, } j \text { is incremented to the next axial station. If they are not equal within the } \\
\text { tolerance, a new value for the hub axial velocity, } c_{a_{1 j+1}} \text { and the whole process is } \\
\text { started again from Step } 1 \text {. }\end{array}$ \\
\hline
\end{tabular}


It is important to note that the initial guess will have serious impact on the convergence of the method. A poor initial guess may lead to non-convergence within the limited number of iterations. For the direct method, the tolerance is measured against the difference between the static pressure calculated from the energy equation using Equation (3.28) and the pressure calculated from radial equilibrium using Equation (3.34). Within CompFlow, the tolerance is set to be one, meaning the difference between the static pressures must be less than one to reach convergence.

(1)

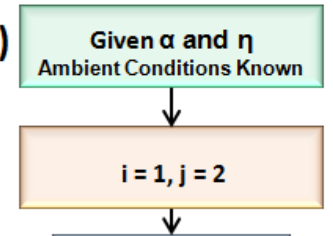

(2)

(3)

(5)

(6)

(7)

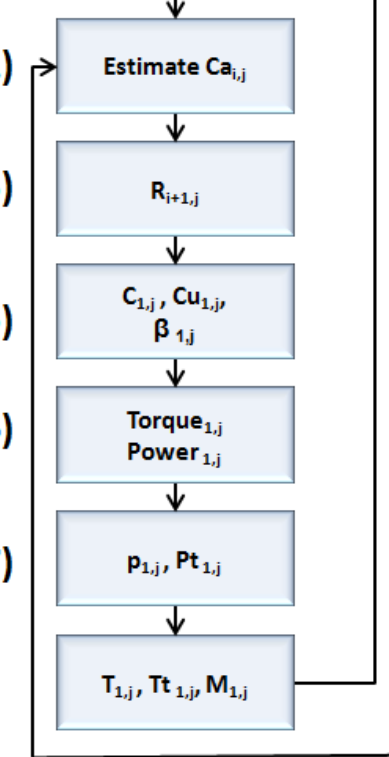

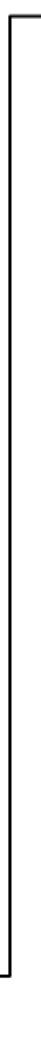
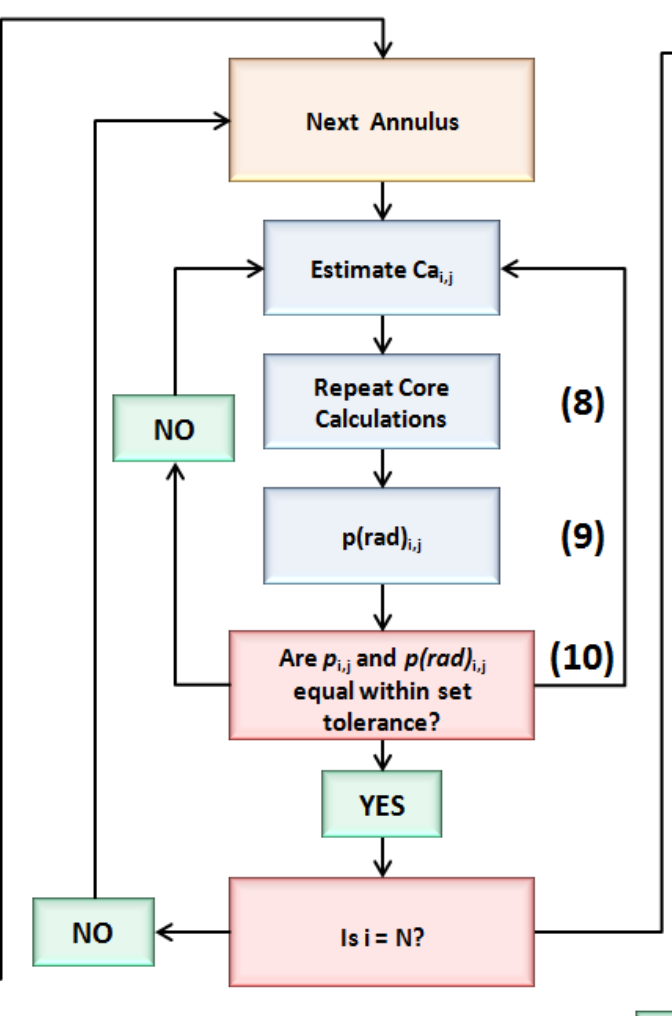

No
(11)

(12)

(13)

Figure 3.6: Flowchart for direct mode calculations 


\subsection{Post-Processing Calculations}

A separate script is used to analyze the results for the different calculation modes of CompFlow. The overall total pressure and temperature ratios for the test compressor case are calculated. The force components for each blade row are also determined. Various plots are also generated that compare velocity components, temperature, Mach number and force components to the percent span of each blade row. The sections below discuss the calculations in more depth. Example plots for test cases are provided in Chapter 4.

\subsubsection{Overall Pressure and Temperature Ratios}

The total pressure ratio and temperature ratio for each blade row is calculated using Equations (3.36) shown below. The average values for the pressure ratio and temperature ratio for each blade row are provided as output by the program.

$$
P R_{j}=\frac{P t_{i j+1}}{P t_{i j}} \quad T R_{j}=\frac{T t_{i j+1}}{T t_{i j}}
$$

The overall pressure ratio and temperature ratio are then calculated using the average value of the radial distribution of total pressure for each blade row.

$$
\begin{gathered}
\pi_{c}=\frac{P t_{s_{-} t o t}}{P t_{1}} \\
\tau_{c}=\pi_{c}^{\gamma-1 / \gamma}
\end{gathered}
$$

Here the subscript $s_{-}$tot refers to the final axial station. The adiabatic and polytropic efficiencies are also calculated using Equations (3.39) and (3.40) respectively: 


$$
\begin{gathered}
\eta_{a d}=\frac{\pi_{c}{ }^{\gamma-1} / \gamma-1}{\tau_{c}-1} \\
\eta_{p c}=\frac{\gamma-1}{\gamma} \frac{\ln \pi_{c}}{\ln \tau_{c}}
\end{gathered}
$$

For plotting purposes, the percent span for each blade row is also calculated using the average radius:

$$
\% \text { span }=\frac{\bar{R}_{i j}-R_{h_{j}}}{R_{t_{j}}-R_{h_{j}}} \cdot 100
$$

\subsubsection{Force Calculations}

The axial and tangential force components are calculated for each blade row. These components will be used to determine the final input values for ROMAC's program RotorSol, which calculates the stability of turbomachines [22]. The control volume used for determining the force components is displayed in Figure 3.7. The spacing $(S)$ between blades can be determined by evenly spacing the total number of blades, $n$. The axial and tangential components of force can be directly calculated from the velocity components and static pressures given by the solution. The force equations shown in Equation (3.44) and (3.45) are provided by Lakshminarayana and account for compressibility [4]. 


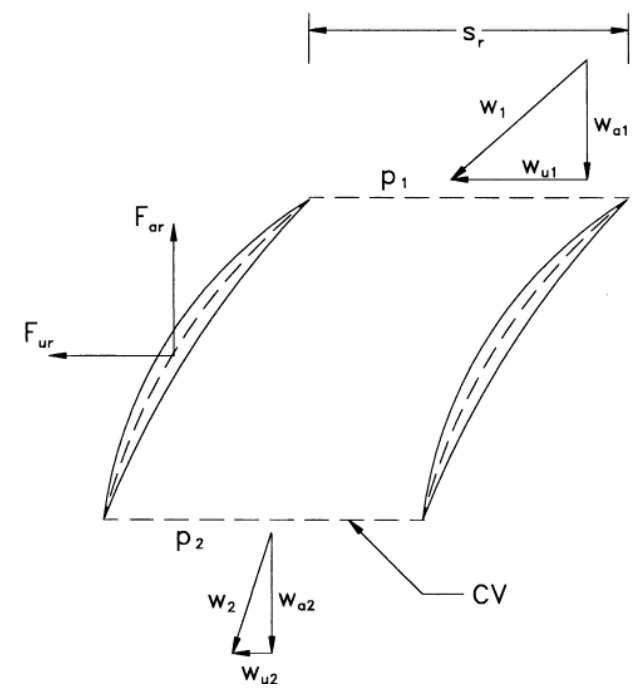

Figure 3.7: Control volume for force component calculations

Geometric parameters used in these calculations are found first. For a blade row, the spacing between blades on the wheel is determined using:

$$
S_{j}=R_{h_{j}} \cdot \frac{2 \pi}{n}
$$

The height of each blade row is determined from:

$$
h_{j}=R_{t_{j}}-R_{h_{j}}
$$

The axial force component is then determined by using the static pressure rise across a blade row and accounting for the axial velocity [4]:

$$
-F_{a_{i j}}=h_{j} \cdot S_{j}\left(p_{i j}-p_{i j-1}\right)+\rho_{j-1} \cdot c_{a_{i j-1}} 2 \cdot S_{j}\left(\frac{\rho_{j-1}}{\rho_{j}}-1\right)
$$

Similarly, the tangential force component, which is based on the difference in tangential velocity components across the blade row, is found using:

$$
F_{u_{i j}}=\rho_{j-1} \cdot h_{j} \cdot S_{j} \cdot c_{a_{i j-1}}\left(c_{u_{i j-1}}-c_{u_{i j}}\right)
$$


Finally, the total force on each stream tube for a blade can be computed by using the axial and tangential force components:

$$
F_{i j}^{2}=F_{u_{i j}}^{2}+F_{a_{i j}}^{2}
$$

\subsubsection{CompFlow to RotorSol axes transformation}

The software package RotorSol consists of Matlab programs that calculate the stability parameters for a specific turbomachinery case, such as critical speed. The two input parameters that CompFlow will provide are the axial force components and the moment due to the tangential force. The axial force components will represent the average axial force for each blade row. The moment about the axial direction is calculated by multiplying the average tangential force by the mean radius:

$$
M_{o_{j}}=F_{u m_{j}} \cdot R_{m_{j}}
$$

The mean radius is computed as the average of the distance between the hub and tip radii at a blade row:

$$
R_{m_{j}}=\frac{1}{2}\left(R_{t_{j}}-R_{h_{j}}\right)
$$

Another point that must be considered is that the axes for CompFlow and RotorSol differ. The axial direction (x) for CompFlow corresponds to the z-axis for RotorSol. The tangential direction (y) for CompFlow lines up with RotorSol's y-axis, and the radial direction $(r)$ with the $x$-axis for RotorSol. The two coordinate systems are 
displayed in Figure 3.8. It is important to make sure that the CompFlow output parameters are correctly entered into RotorSol.

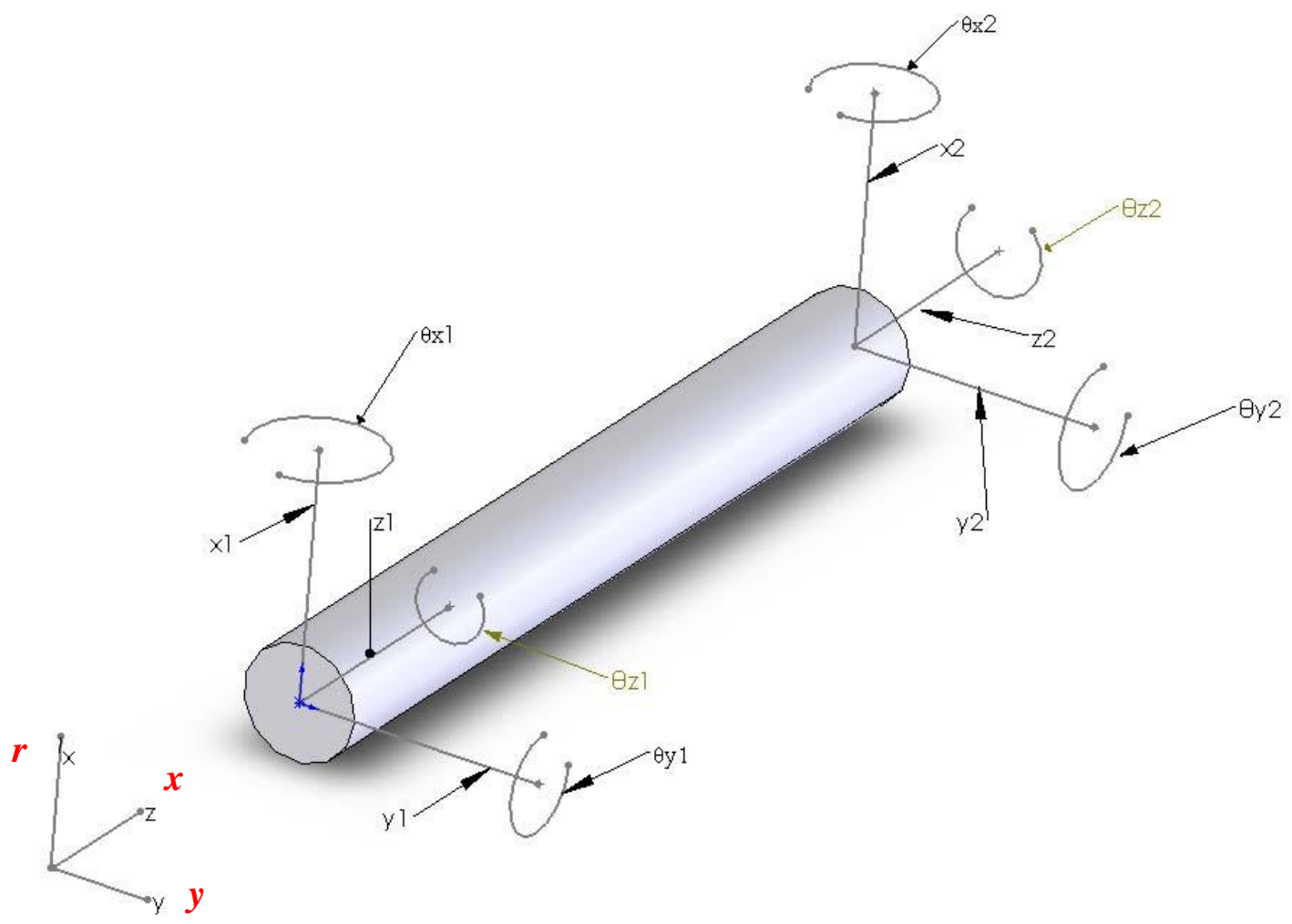

Figure 3.8: RotorSol and CompFlow (red) coordinate systems [22] 


\section{Chapter 4}

\section{Verification and Validation}

\subsection{Introduction to Verification and Validation}

Before a code can be released, confidence in the accuracy of the calculations must first be reached. This step is done through verification and validation. Verification addresses the accuracy of the model by looking at the mathematics involved. Validation addresses the appropriateness of the given model itself in dealing with the physics of the problem [23]. Validation mainly involves comparing the numerical solution to experimental data. The following chapter will discuss the verification of CompFlow using a simple compressor case provided by Flack, and the validation of CompFlow using open source NASA compressor cases.

\subsection{Verification using Flack's Single Stage Compressor}

The verification step of this process will ensure that the program is running as intended, i.e. by debugging the code [24]. The analysis of intermediate results was also utilized to ensure the code was running properly. A simple compressor case was used to test the coding of CompFlow. The input parameters and results from a single-stage compressor case as provided by Flack are further described below [5]. 


\subsubsection{Flack Compressor Geometry}

The test case that was used for verification of the code was a single-stage compressor, which includes an inlet guide vane (IGV), rotor, and stator. This case was selected for verification because the geometry is relatively simple, and results were provided by Flack's streamline method. A schematic of this compressor is shown in Figure 4.1 below. The schematic shows that four axial stations are used by the code, one in front of the IGV to represent ambient or freestream conditions, and one behind each blade row respectively. Although the schematic only shows four annuli, nine were actually used in the calculations.

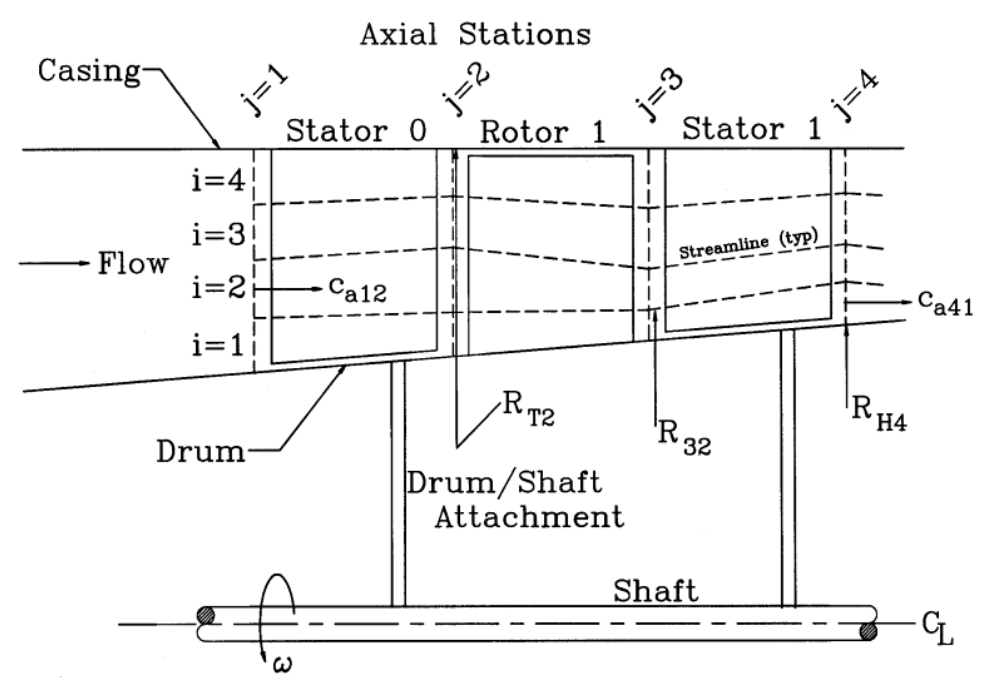

Figure 4.1: Geometry for Flack's single stage compressor [5]

Table 4.1 below summarizes the input parameters for this test case. For this case, the IGV, rotor, and stator blades have the same dimensions, so only one hub radius and tip radius is listed below. The efficiency distribution is the same for each blade row, with a constant value of 0.90 in the middle and reduced values of 0.87 at the hub and tip radii. 
The absolute flow angles at the exit of each blade row are also specified with the IGV and the stator having the same angle of $11^{\circ}$ and the rotor with $34.2^{\circ}$. These values are constant from hub to tip for each corresponding blade row. As described before, either the static pressure or the total pressure and temperature at the inlet can be specified to run both the indirect and direct modes of calculation. For this case, the static temperature has been stated, along with the density and mass flow rate. Finally, the number of annuli and axial stations used are also listed at the bottom of the table.

Table 4.1: Input parameters for Flack compressor

\begin{tabular}{|c|c|c|}
\hline \multicolumn{3}{|c|}{ Flack Compressor Example } \\
\hline \multicolumn{3}{|c|}{ Geometry } \\
\hline Hub Radius & 0.16 & $\mathrm{~m}$ \\
\hline Tip Radius & 0.1955 & $\mathrm{~m}$ \\
\hline \multirow{2}{*}{ Rotational Speed } & 12000 & $\mathrm{rpm}$ \\
\hline & 1256.6 & $\mathrm{rad} / \mathrm{s}$ \\
\hline \multicolumn{3}{|c|}{ Initial Parameters } \\
\hline Efficiency & $\begin{array}{l}0.87 \\
0.90 \\
\end{array}$ & $\begin{array}{c}\text { hub,tip } \\
\text { midspan }\end{array}$ \\
\hline IGV Exit Angle & 11 & degrees \\
\hline Rotor Exit Angle & 34.2 & degrees \\
\hline Stator Exit Angle & 11 & degrees \\
\hline \multicolumn{3}{|c|}{ Ambient Conditions } \\
\hline Ambient Pressure & 97900 & $\mathrm{~Pa}$ \\
\hline Ambient Density & 1.21 & $\mathrm{~kg} / \mathrm{m}^{3}$ \\
\hline Mass Flow Rate & 11.3 & $\mathrm{~kg} / \mathrm{s}$ \\
\hline \multicolumn{3}{|c|}{ SLA Parameters } \\
\hline Number of Annuli & 9 & \\
\hline Number of Axial Stations & 4 & \\
\hline
\end{tabular}

\subsubsection{Flack Compressor Results}

Since "raw experimental" data was not provided for this compressor case, the comparison is done in a more qualitative way. As stated before, this case was used to 
verify the code was working properly whenever changes were made. The first check for each occurrence was to compare the axial velocity distributions to the ones provided by Flack. This comparison is shown below in Figure 4.2. Although the values for the axial velocities vary towards the hub and tip region between the two figures, the shapes of the profiles are very similar. A simple comparison of the two graphs shows that the difference between the axial velocities is less than 3\%, which is acceptable. This difference in value is largely attributed to the fact that Flack utilizes the simple radial equilibrium expression as seen in Equation (3.2), whereas CompFlow uses the modified radial equilibrium expression shown in Equation (3.10). As the values fall within the same range, the code is deemed to not contain major coding errors.
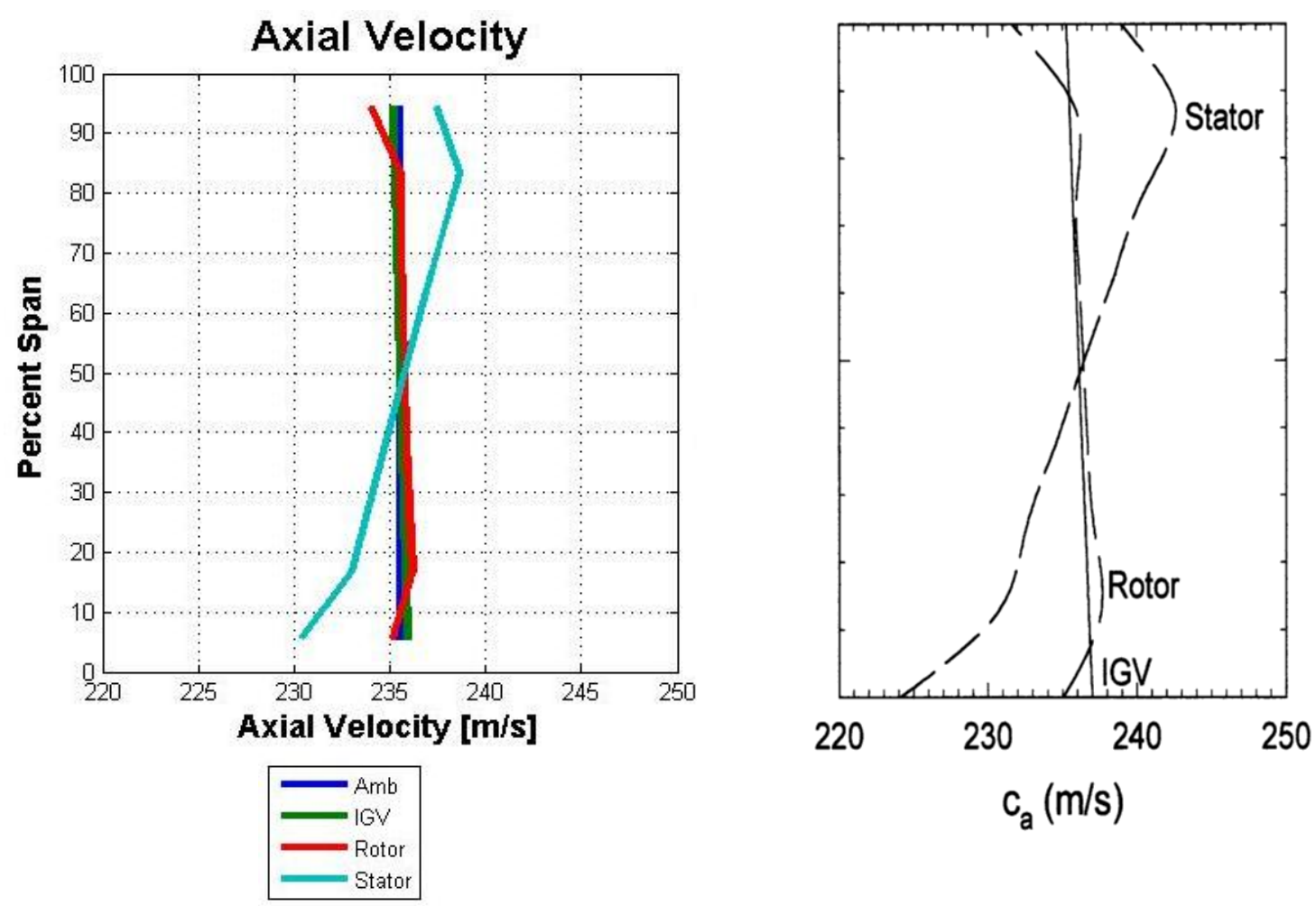

(a)

(b)

Figure 4.2: Axial Velocity comparison for CompFlow (a) and Flack (b) [5] 
Another simple comparison involves the static pressures and total pressures for the two calculations. Table 4.2 below summarizes this study. The values from CompFlow represent the average of the radial distribution of the static and total pressures for the comparison stations listed. The values from Flack were estimated from a graph. As seen in the table, the values match within $1 \%$. Therefore, the code can be deemed to be working. The next section discusses the validation of CompFlow.

Table 4.2: Comparison of static and total pressures for Flack compress
\begin{tabular}{|l|c|c|}
\hline & Comp Flow & Flack \\
\hline & Static Pressure (MPa) \\
\hline Ambient & 0.098 & 0.099 \\
\hline Rotor & 0.110 & 0.110 \\
\hline Stator & 0.124 & 0.123 \\
\hline & \multicolumn{2}{|c|}{ Total Pressure (MPa) } \\
\hline Inlet & 0.132 & 0.131 \\
\hline Exit & 0.159 & 0.161 \\
\hline
\end{tabular}

\subsection{Validation Using NASA Stage 37}

The following section will discuss the validation of CompFlow, in which computational results for a test case are compared to experimental data. The purpose of this study is to determine whether the governing equations chosen present a good model of the flow physics. The compressor case chosen for this process is the open source NASA Stage 37, a single stage compressor.

Stage 37 was chosen as a CFD test case in 1992 by the turbomachinery committee of the International Gas Turbine Institute (IGTI) [25]. The compressor was presented as a "blind" test case, meaning no experimental data was provided. Since then, Stage 37 has become a standard for validating CFD codes due to the plentiful amount of experimental 
data provided with the geometry. For CompFlow, this stage is used to check the validity of the flow model, to conduct an annuli sensitivity study, and to perform an off-design study.

\subsubsection{Stage 37 Geometry}

Stage 37 consists of a single row of rotors and stators respectively. All experimental data associated with Stage 37 has been provided by Reid and Moore [26]. The rotor and stator blades have varying radii, as shown in the table. The rotational speed shown below is representative of $100 \%$ design speed. Later, this value will be varied for an off-design study. The number of annuli used in this case is 11 , which equals the number of radial data points provided by Reid and Moore.

Table 4.3: Input parameters for NASA Stage 37

\begin{tabular}{|c|c|c|c|}
\hline \multicolumn{4}{|c|}{$\begin{array}{c}\text { NASA Stage } 37 \\
\text { Geometrv }\end{array}$} \\
\hline & $\begin{array}{c}\text { Hub } \\
(\mathrm{m})\end{array}$ & $\begin{array}{l}\text { Tip } \\
\text { (m) }\end{array}$ & Number of Blades \\
\hline Ambient & 0.178 & 0.252 & \\
\hline Rotor & 0.187 & 0.245 & 36 \\
\hline Stator & 0.192 & 0.240 & 46 \\
\hline \multicolumn{4}{|c|}{ Initial Parameters } \\
\hline \multirow{2}{*}{ Rotational Speed } & \multicolumn{2}{|c|}{17188.7} & $\mathrm{rpm}$ \\
\hline & \multicolumn{2}{|c|}{1800.0} & $\mathrm{rad} / \mathrm{s}$ \\
\hline \multicolumn{4}{|c|}{ Ambient Conditions } \\
\hline Ambient Pressure & \multicolumn{2}{|c|}{101330} & $\mathrm{~Pa}$ \\
\hline Ambient Density & \multicolumn{2}{|c|}{1.225} & $\mathrm{~kg} / \mathrm{m}^{3}$ \\
\hline Mass Flow Rate & \multicolumn{2}{|c|}{20.2} & $\mathrm{~kg} / \mathrm{s}$ \\
\hline \multicolumn{4}{|c|}{ SLA Parameters } \\
\hline Number of Annuli & \multicolumn{2}{|c|}{11} & \\
\hline Number of Axial Stations & \multicolumn{2}{|c|}{3} & \\
\hline
\end{tabular}


The table below displays the radial distributions for the exit angles and efficiency for each blade row. This information is necessary for the direct mode of calculations. These values will be used for various studies conducted. Since a moderate amount of data is necessary to run these calculations, a graphical user interface (GUI) was developed to aid in the calculation process. Detailed instructions on running and navigating the CompFlow GUI are provided in Appendix C.

Table 4.4: NASA Stage 37 Row Exit Conditions

\begin{tabular}{|c|c|c|c|}
\hline \multicolumn{5}{|c|}{ Row Exit Conditions } \\
\hline Annulus Number & $\begin{array}{c}\text { Rotor Exit Angle } \\
(\mathbf{d e g})\end{array}$ & $\begin{array}{c}\text { Stator Exit Angle } \\
(\mathbf{d e g})\end{array}$ & Efficiency \\
\hline Hub & 48.3 & 12.5 & 0.929 \\
\hline $\mathbf{2}$ & 48.6 & 12.3 & 0.927 \\
\hline $\mathbf{3}$ & 48.8 & 12.2 & 0.924 \\
\hline $\mathbf{4}$ & 48.9 & 12. & 0.922 \\
\hline $\mathbf{5}$ & 48.6 & 11.6 & 0.917 \\
\hline $\mathbf{6}$ & 48.5 & 11.1 & 0.894 \\
\hline $\mathbf{7}$ & 49.1 & 10.8 & 0.854 \\
\hline $\mathbf{8}$ & 50 & 10.6 & 0.818 \\
\hline $\mathbf{9}$ & 50.1 & 10.6 & 0.809 \\
\hline $\mathbf{1 0}$ & 50.4 & 10.6 & 0.797 \\
\hline Tip & 50.7 & 10.6 & 0.786 \\
\hline
\end{tabular}

The ambient velocity used for this case was determined by the code using Equation (3.16), with a value of $163.8 \mathrm{~m} / \mathrm{s}$. Since this is a single stage, the flow will be modeled as incompressible regardless of what option is chosen on the GUI. At this time, no blockage was selected to be present in the calculations.

\subsubsection{Stage 37 Results}

The outputs of CompFlow includes plots of the total temperature and pressure rise across each blade row (Figure 4.4), axial velocity and Mach number (Figure 4.3), and 
blade force distributions (Figure 4.5). A schematic of the streamline movement throughout the compressor is also included. The following studies will focus on agreement between the total pressure and temperature ratios as that is the experimental data available for comparison. Example output plots for the Stage 37 are shown below.

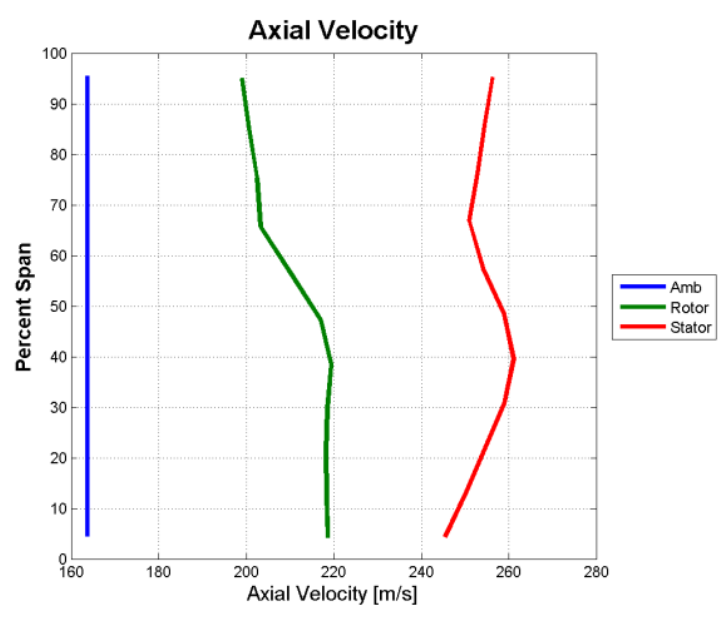

(a)

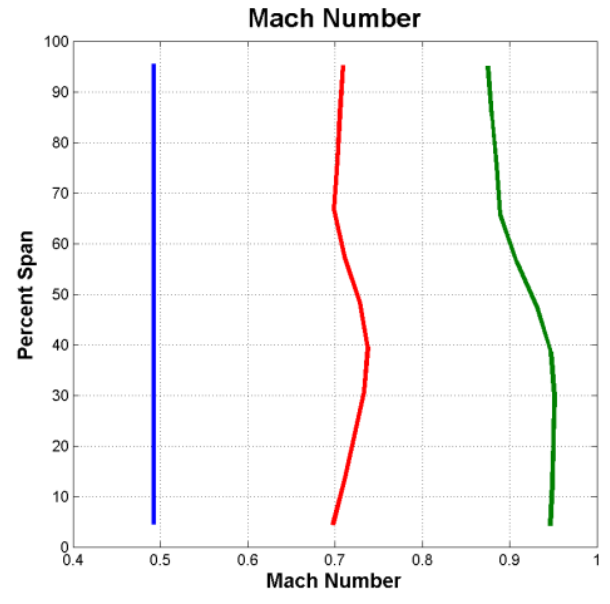

(b)

Figure 4.3: Stage 37 results for axial velocity (a) and Mach number (b)

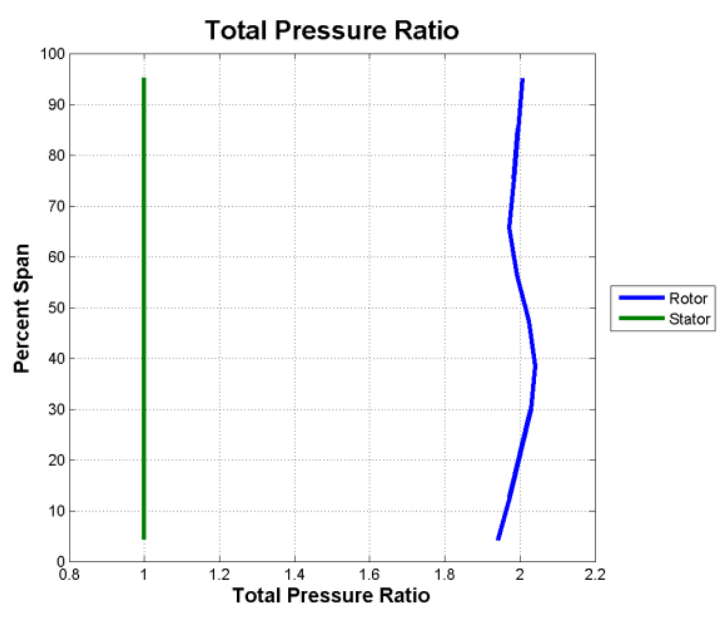

(a)

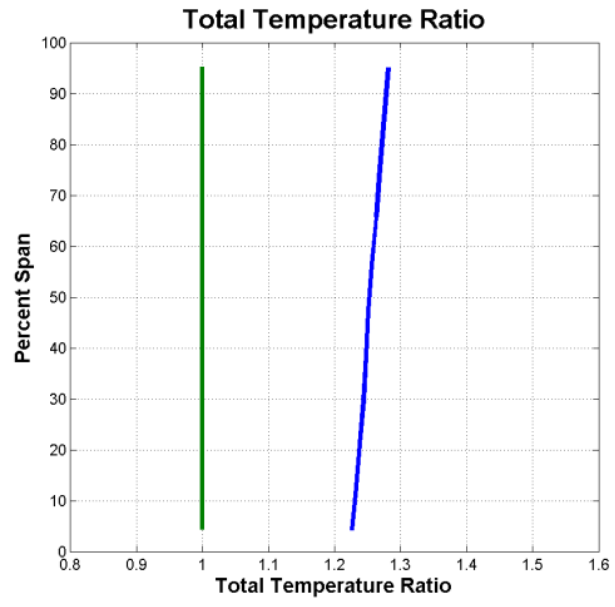

(b)

Figure 4.4: Stage 37 results for total pressure ratio (a) and total temperature ratio (b) 


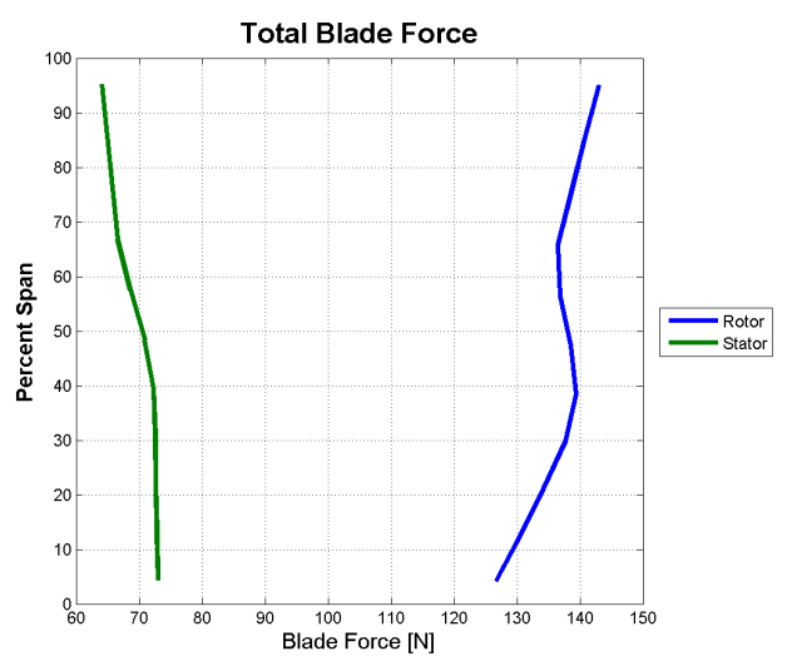

(a)

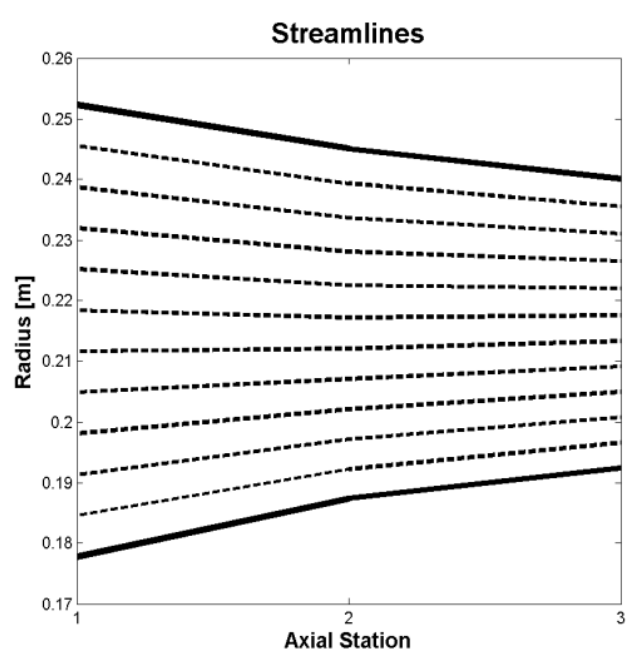

(b)

Figure 4.5: Stage 37 results for blade force (a) and streamlines (b)

Figure 4.3 shows the plots for axial velocity and Mach number for Stage 37 . The axial velocity is seen to increase across the blade rows, but inspection of the total absolute velocity shows that it decreases over the stator. This behavior follows the trend as described in Table 3.1. Figure 4.4 shows the total pressure ratio and total temperature ratio over the blade rows. These graphs also follow the trends described in Table 3.1, where the ratio increases over the rotor, but is constant for the stator as seen by a ratio of unity. The blade forces and streamlines are presented in Figure 4.5. The forces were determined for the rotor wheel having 36 blades and the stator with 46 blades. Note that the streamline plots do not account for the axial distance between blade rows.

Direct comparisons with experimental data can be done for this case. The first analysis done was for the total pressure ratio and total temperature ratio, as shown in Figure 4.6. The experimental data is portrayed by the red data points, and the CompFlow results are shown by solid lines. As the pressure and temperature ratios for the stator are equal to unity for the stator blade rows, it is more meaningful to compare the rotor 
computational and experimental data. The total temperature ratio appears to match well with the experimental data. The total pressure ratio varies more, but the shapes of the profiles are similar to the experimental data.

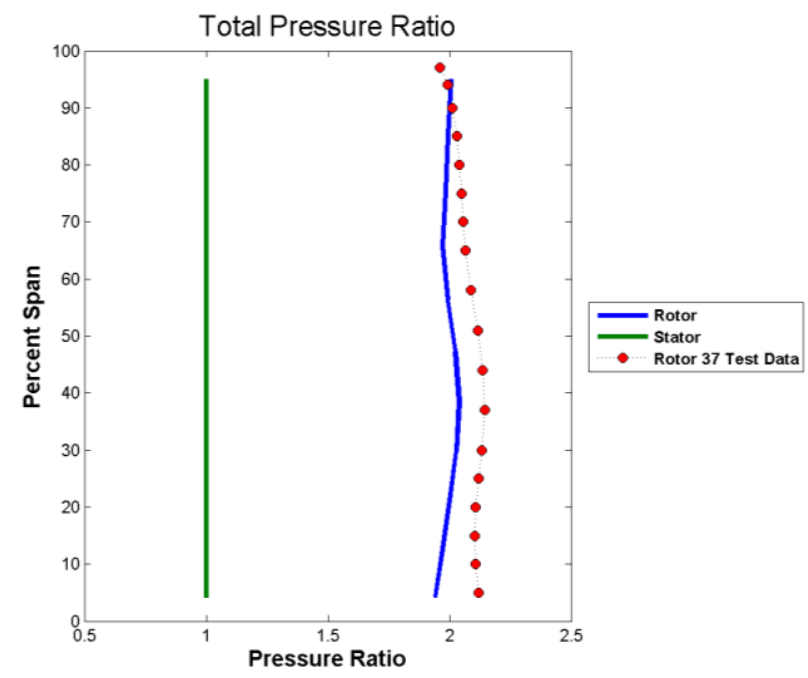

(a)

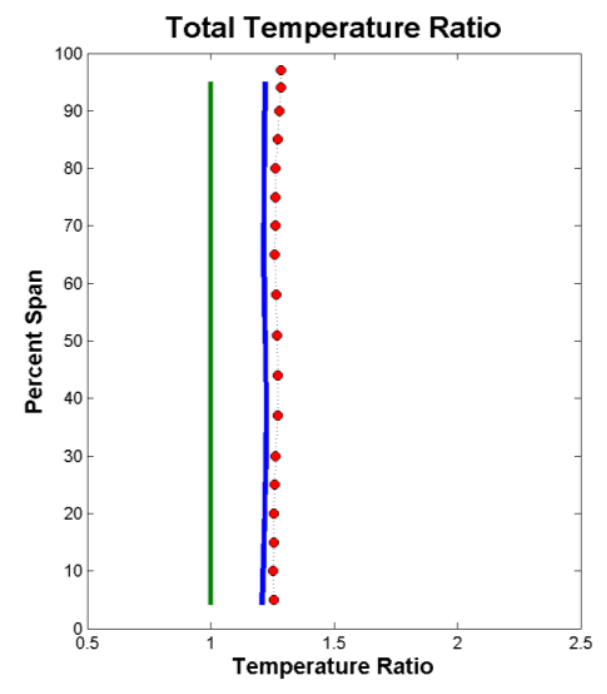

(b)

Figure 4.6: Comparison with experimental data for Stage 37

More comparisons can be made by examining the overall compressor parameters. The values of interest are the stage pressure ratio, temperature ratio, adiabatic efficiency and polytropic efficiency. These comparisons are summarized in Table 4.5 below, which yield that the percent difference between the overall compressor values is less than $3 \%$. Therefore, the modeling of the flow physics within CompFlow represents good matching for the associated experimental data. Although the radial distributions vary slightly between the results, the overall compressor parameters are captured well by the program. These radial variations occur more strongly towards the hub and tip region, which may be associated with end-wall effects that are not completely modeled by the code. 
Table 4.5: Comparison of Overall Compressor Data

\begin{tabular}{|c|c|c|c|}
\hline \multicolumn{4}{|c|}{ NASA Stage 37 } \\
\hline & CompFlow & NASA Data & \% Difference \\
\hline Stage PR & 1.996 & 2.050 & 2.63 \\
\hline Stage TR & 1.253 & 1.270 & 1.34 \\
\hline Adiabatic Efficiency & 0.867 & 0.842 & 2.97 \\
\hline Polytropic Efficiency & 0.879 & 0.857 & 2.57 \\
\hline
\end{tabular}

\subsubsection{Annuli Sensitivity Study}

In terms of computational fluid dynamics, the distribution of annuli and axial stations represents the "mesh" of the geometry. For CFD cases, it is important to control the size of the mesh in order to optimize computational power and run-time with solution accuracy. Within CompFlow, the mesh size is determined by the number of annuli. Therefore, a sensitivity study on the number of annuli is conducted to determine the limits for the program using Stage 37.

For this case, the mesh cannot accurately be increased as there is not enough data. Therefore, the study presented in this section will determine the lower limit for the number of annuli. The mesh starting with 11 annuli was systematically reduced until it approached the input for a mean-line code, which is three data points. This reduction affected the values for the exit flow angle and efficiency. Values for exit angles and efficiency for each case study are presented in Table 4.6 and Table 4.7. 
Table 4.6: Rotor Exit Flow Angles for Senstivity Study

\begin{tabular}{|c|c|c|c|c|c|c|c|c|c|c|}
\hline Annulus & \multicolumn{2}{|c|}{ Case 1 } & \multicolumn{2}{c|}{ Case 2 } & \multicolumn{2}{c|}{ Case 3 } & \multicolumn{2}{c|}{ Case 4 } & \multicolumn{2}{c|}{ Case 5 } \\
\hline & Rotor & Stator & Rotor & Stator & Rotor & Stator & Rotor & Stator & Rotor & Stator \\
\hline $\mathbf{1}$ & 48.30 & 12.30 & 48.30 & 12.30 & 48.30 & 12.30 & 48.30 & 12.30 & 48.30 & 12.30 \\
\hline $\mathbf{2}$ & 48.60 & 12.30 & 48.70 & 12.25 & 48.78 & 12.18 & 48.79 & 12.06 & 48.50 & 11.10 \\
\hline $\mathbf{3}$ & 48.80 & 12.20 & 48.85 & 12.10 & 48.80 & 11.95 & 48.50 & 11.10 & 50.70 & 10.60 \\
\hline $\mathbf{4}$ & 48.90 & 12.00 & 48.75 & 11.80 & 48.50 & 11.10 & 49.98 & 10.63 & & \\
\hline $\mathbf{5}$ & 48.60 & 11.60 & 48.50 & 11.10 & 49.80 & 10.65 & 50.70 & 10.60 & & \\
\hline $\mathbf{6}$ & 48.50 & 11.10 & 49.55 & 10.70 & 50.15 & 10.60 & & & & \\
\hline $\mathbf{7}$ & 49.10 & 10.80 & 50.05 & 10.60 & 50.70 & 10.60 & & & & \\
\hline $\mathbf{8}$ & 50.00 & 10.60 & 50.25 & 10.60 & & & & & & \\
\hline $\mathbf{9}$ & 50.10 & 10.60 & 50.70 & 10.60 & & & & & & \\
\hline $\mathbf{1 0}$ & 50.40 & 10.60 & & & & & & & & \\
\hline $\mathbf{1 1}$ & 50.70 & 10.60 & & & & & & & & \\
\hline
\end{tabular}

Table 4.7: Efficiency Distributions for Sensitivity Study

\begin{tabular}{|l|l|l|l|l|l|}
\hline \multicolumn{1}{|c|}{ Annulus } & \multicolumn{1}{c|}{ Case 1 } & \multicolumn{1}{c|}{ Case 2 } & \multicolumn{1}{c|}{ Case 3 } & \multicolumn{1}{c|}{ Case 4 } & Case 5 \\
\hline 1 & 0.929 & 0.929 & 0.929 & 0.929 & 0.929 \\
\hline 2 & 0.927 & 0.926 & 0.924 & 0.923 & 0.894 \\
\hline 3 & 0.924 & 0.923 & 0.921 & 0.894 & 0.786 \\
\hline 4 & 0.922 & 0.920 & 0.894 & 0.8165 & \\
\hline 5 & 0.917 & 0.894 & 0.825 & 0.786 & \\
\hline 6 & 0.894 & 0.836 & 0.808 & & \\
\hline 7 & 0.954 & 0.814 & 0.786 & & \\
\hline 8 & 0.818 & 0.803 & & & \\
\hline 9 & 0.809 & 0.786 & & & \\
\hline 10 & 0.797 & & & & \\
\hline 11 & 0.786 & & & & \\
\hline
\end{tabular}

The graphical results of the study are presented in Figure 4.7 below. Only the total pressure ratio for each case is plotted as it better exhibits the variation of data due to annuli. For the first case with 11 annuli, it is clear that the profile shape is smooth and follows the data trend for the experimental values. As the number of annuli is decreased, the profile shape becomes more jagged. The study also shows that decreasing the number of annuli shortens the span the data covers. This means valuable information near the hub and tip is not obtained for coarser meshes as seen in Figure 4.7e. 
Although larger meshes were not considered due to the lack of experimental data, a good estimate of an optimal annuli number can be determined. The recommendation is to set the number of annuli to a value between 9 and 15 for optimal performance. This range is predicted to allow for sufficient accuracy while decreasing the runtime. Increasing the number of annuli above 21 would significantly increase the runtime while not necessarily increasing the accuracy of the solution. This would need to be tested more thoroughly in the future for confirmation.

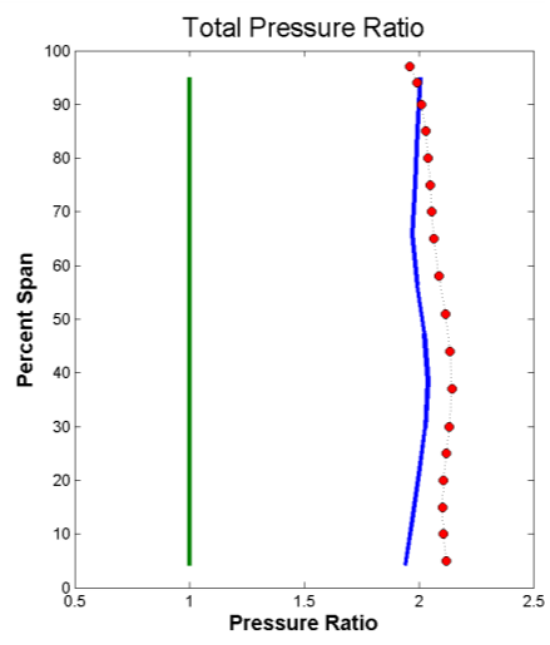

(a)

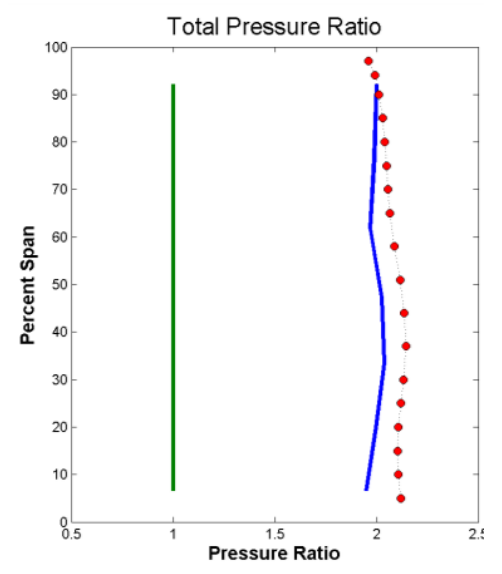

(c)

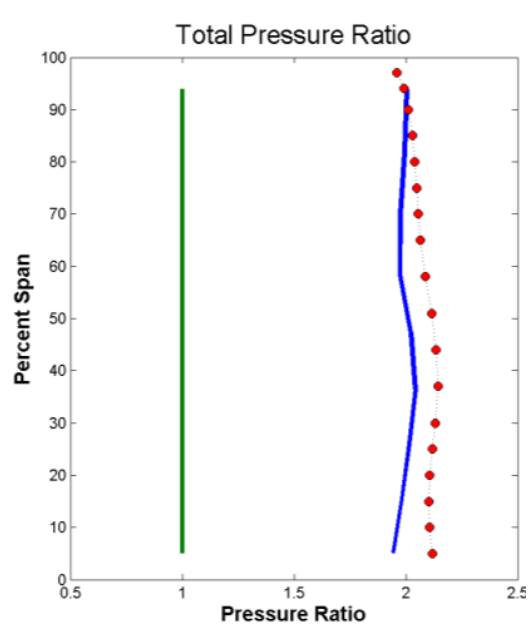

(b)

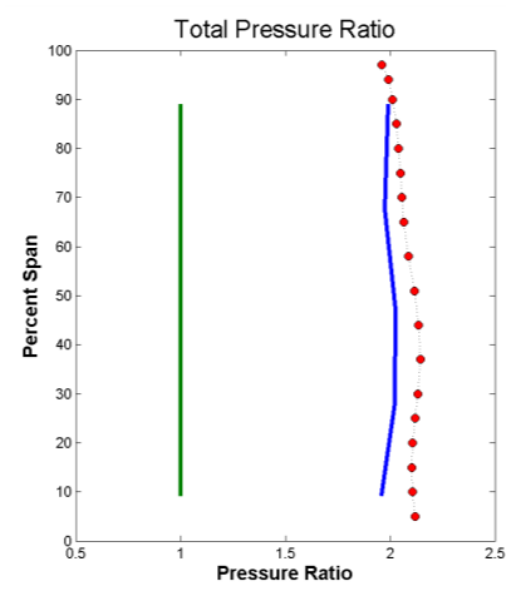

(d) 

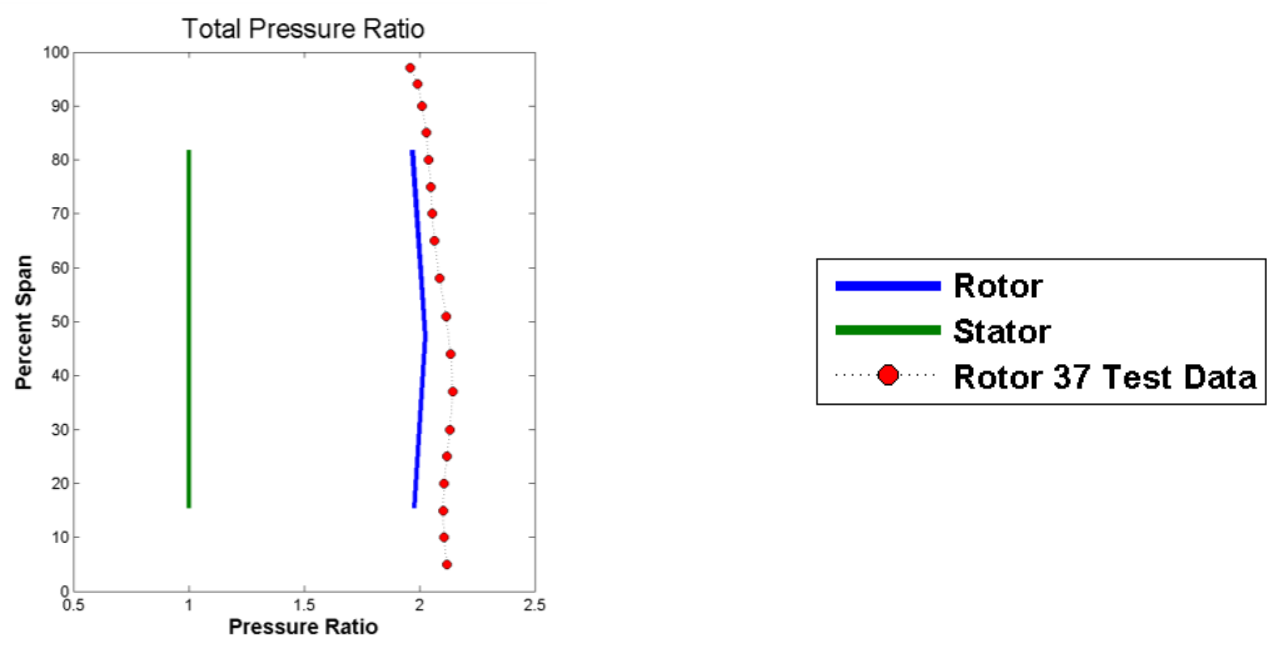

(e)

Figure 4.7: Results for Stage 37 sensitivity study with (a) 11 annuli, (b) 9 annuli, (c) 7 annuli, (d) 5 annuli and (e) 3 annuli

\subsubsection{Off-Design Study}

This section presents the final study conducted on Stage 37. An "off-design" study was conducted in which the design rotational speed the compressor normally operates is decreased. The ability to accurately predict off-design characteristics would be useful for rotordynamic purposes. The operational speeds tested were from $100 \%$ to $50 \%$ in increments of $10 \%$. The number of annuli for all the cases was set to 11 , which is the same as the base case.

The figures below show that as the rotational speed is decreased, the matching of the computational and experimental values becomes increasingly poor. Although the temperature ratio matches well, the total pressure ratio matching becomes significantly worse as the speed is decreased. Therefore, CompFlow at this time cannot accurately predict "off-design" characteristics for a compressor case. Future work should include 
further analysis of this capability. One important point is that in commercial jet engine compressors the stator blades often have the capability to vary the geometry, meaning the exit flow angle is changed with varying operating conditions. Thus, angles specified for $100 \%$ rotational speed may not be optimal for a lower rotational speed. A future study would include changing the exit flow angle with decreasing rotational speed.
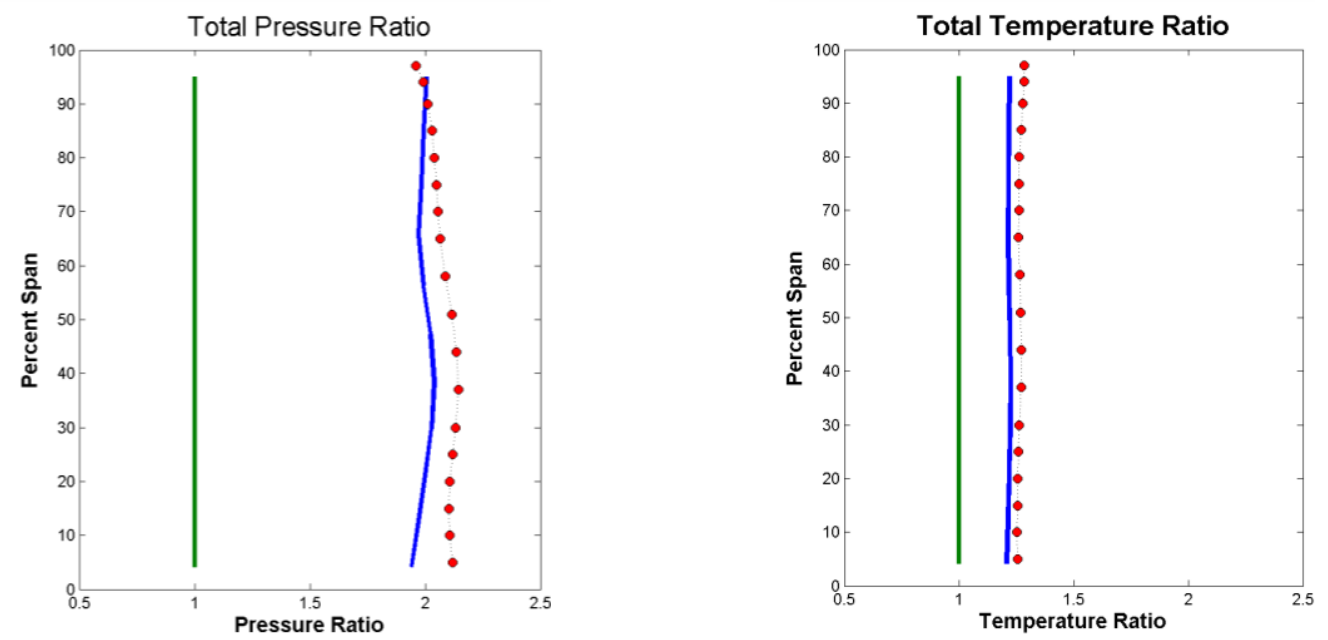

Figure 4.8: Off-design study for $100 \%$ RPM
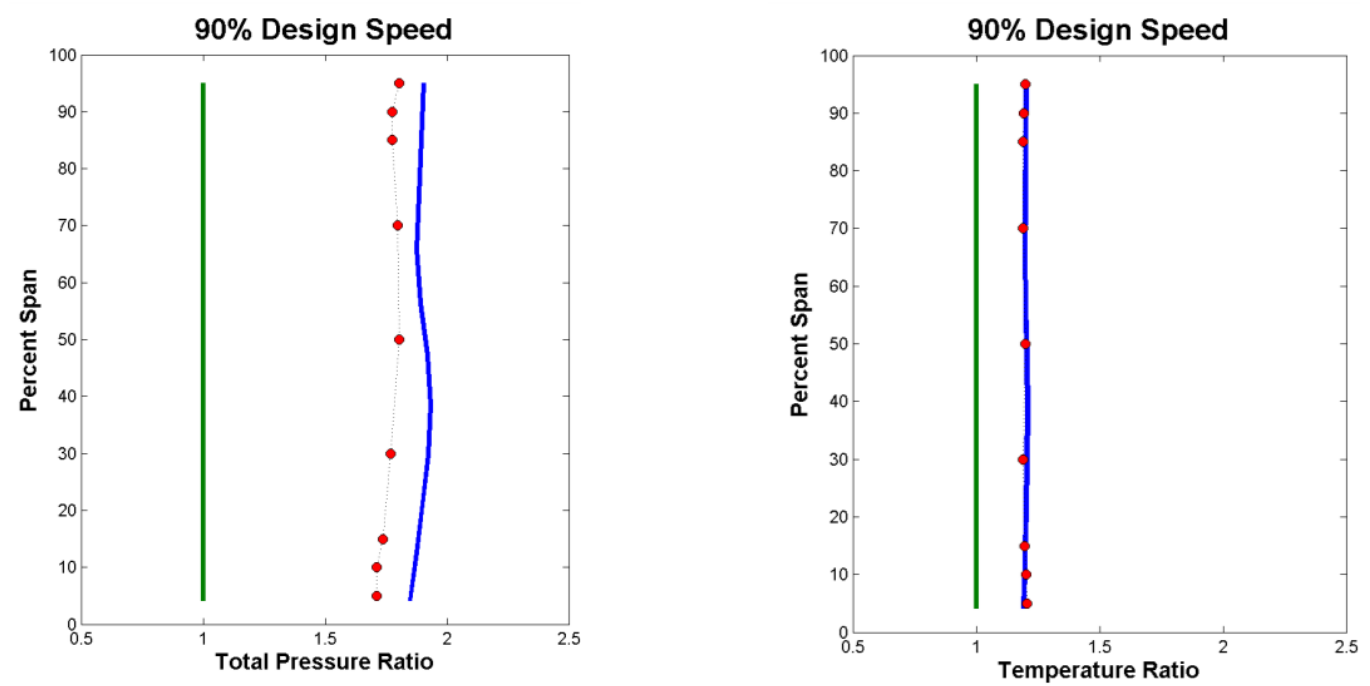

Figure 4.9: Off-design study for 90\% RPM 

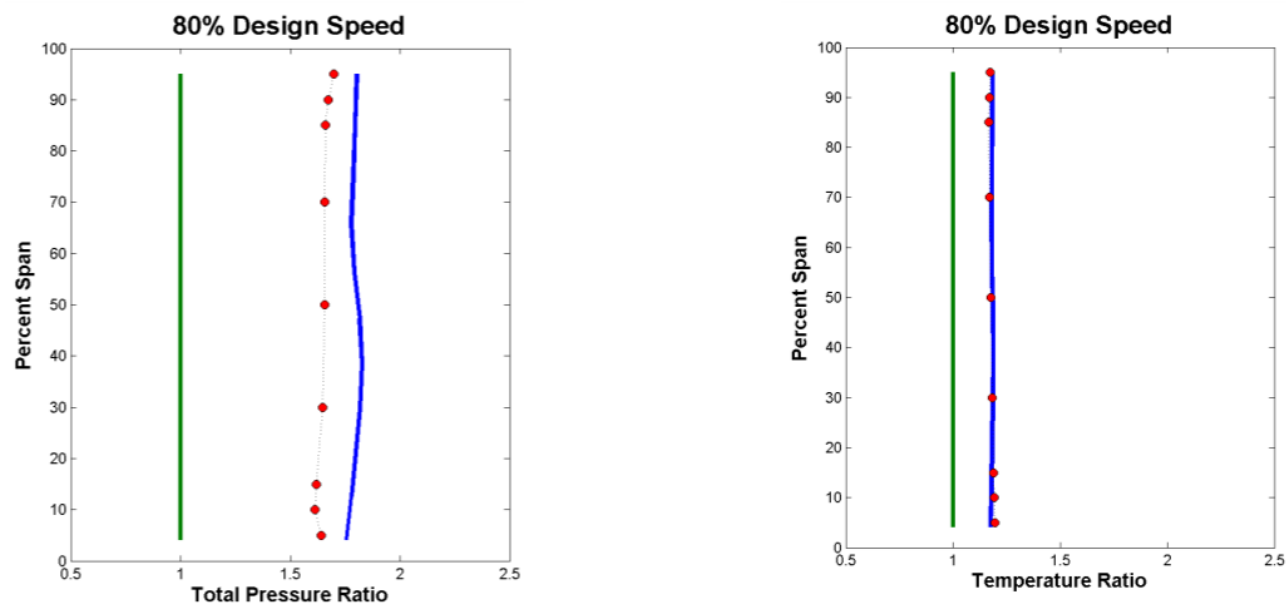

Figure 4.10: Off-design study for $80 \%$ RPM
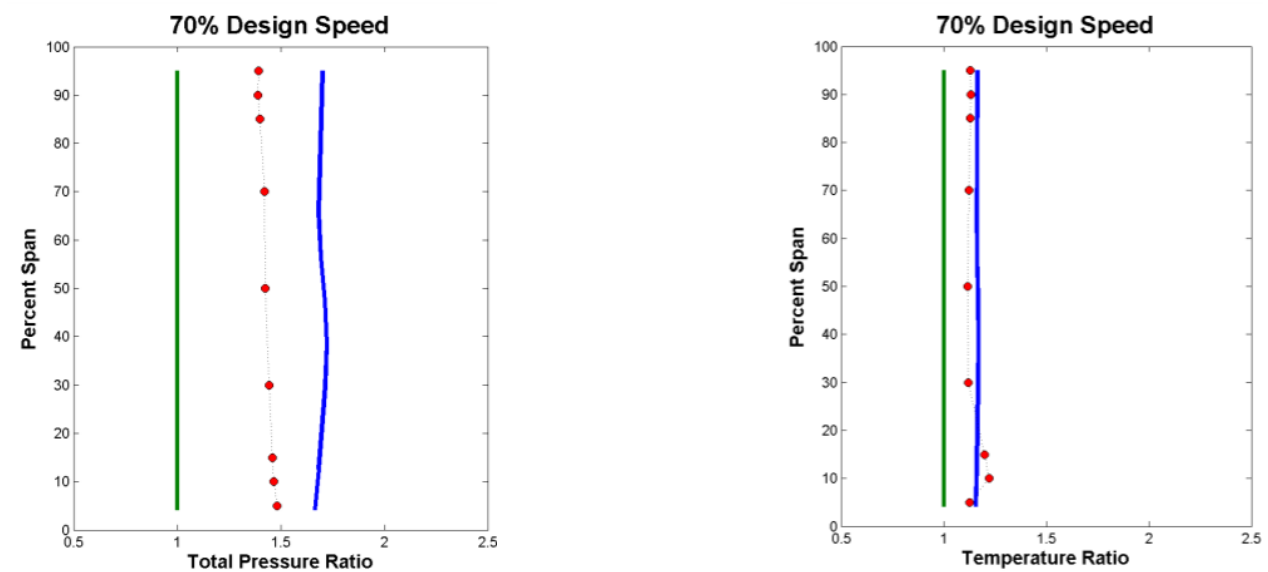

Figure 4.11: Off-design study for 70\% RPM
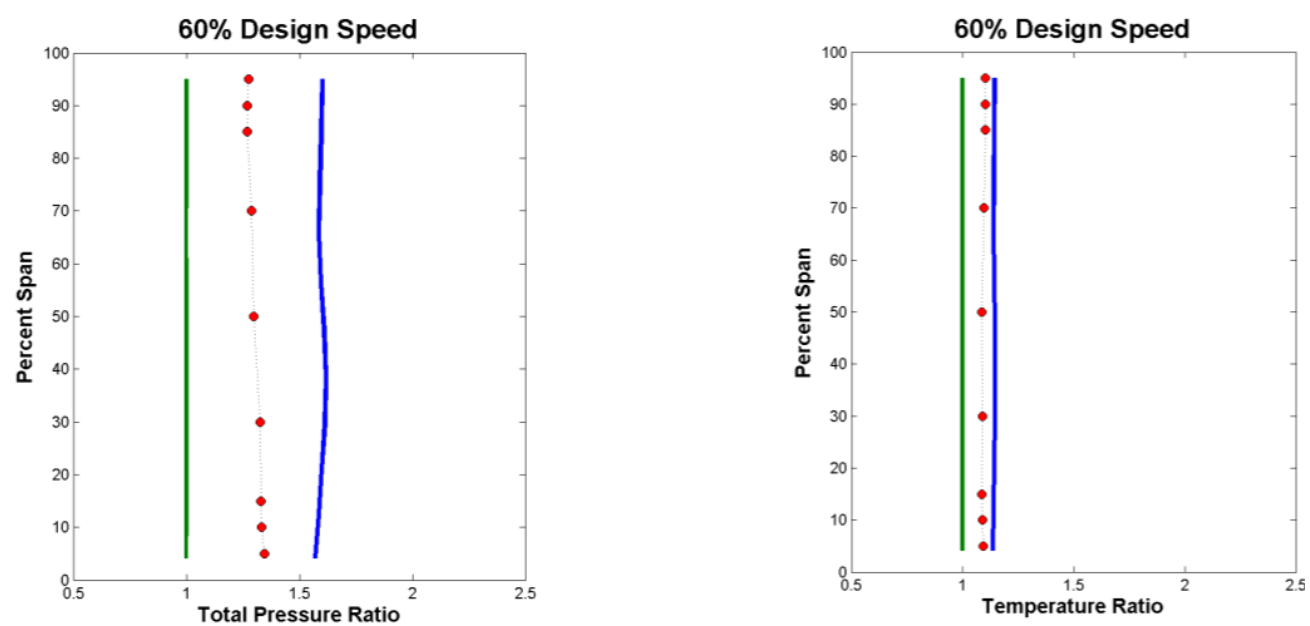

Figure 4.12: Off-design study for 60\% RPM 

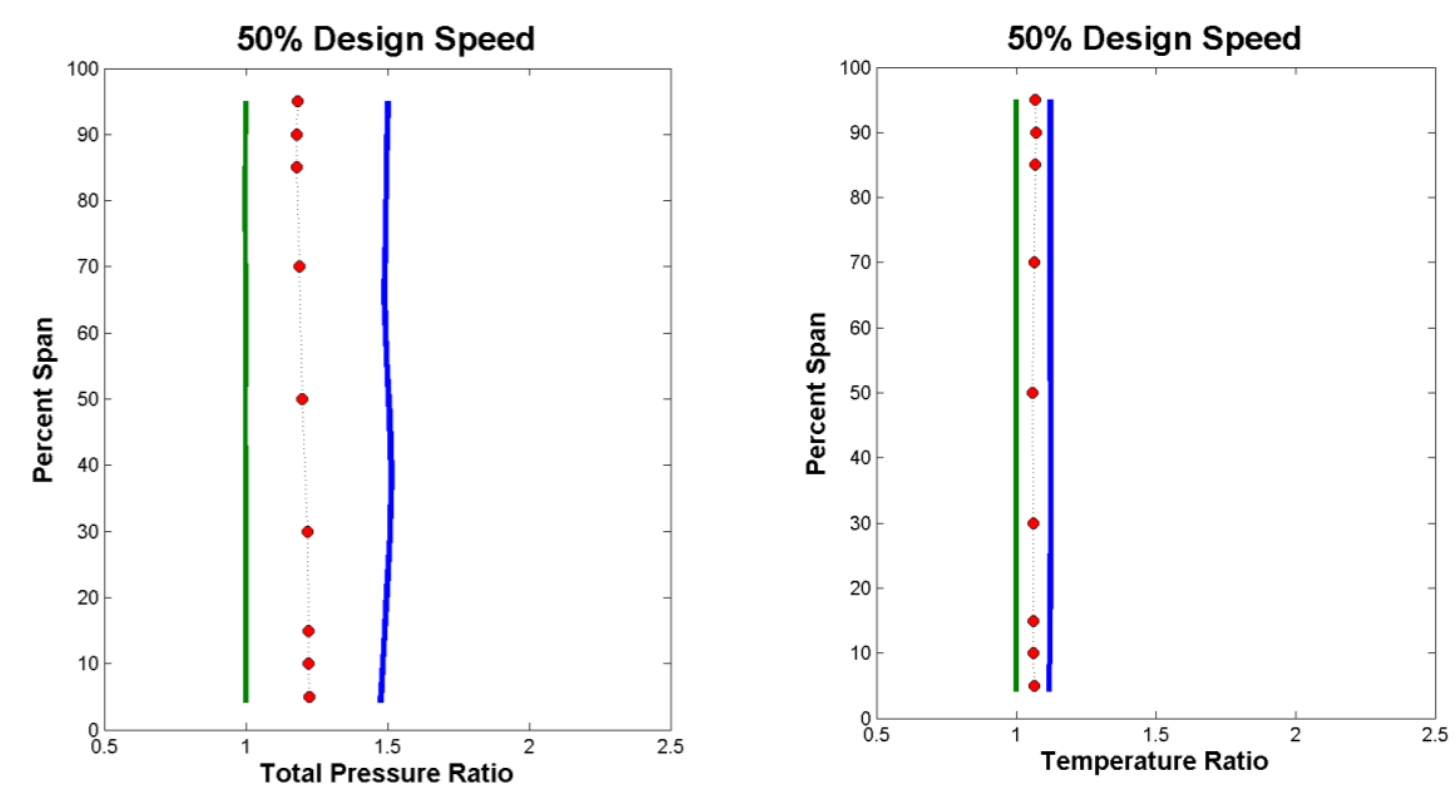

Figure 4.13: Off-design study for 50\% RPM

\subsection{Validation Using NASA Compressor 74A}

Another compressor case that will be used for validation is the NASA 74A compressor. This case was tested experimentally at NASA Lewis in 1986, and consists of a five stage compressor with an IGV at the inlet. As the geometric data was limited to the inlet portion of the compressor, only the first three stages and the IGV will be analyzed in CompFlow. All geometric data for NASA 74A was provided by Steinke [27]. This case is significant for the validation process, as it presents an example of a multi-stage compressor. As there can be compounding effects with multiple stages, matching data across stages is crucial for proper validation. The geometry inputs for this case are described further in the next section. 


\subsubsection{NASA 74A Geometry}

A schematic of the compressor geometry is shown below in Figure 4.14. Note that this case has strong curvature along the hub wall. The amount of data provided allows for the number of annuli to be set to 13 , which is within the optimal range as determined by the sensitivity study. The radial efficiency distribution for all the blade rows is shown in Table 4.8, and the geometry inputs for this case are summarized in.

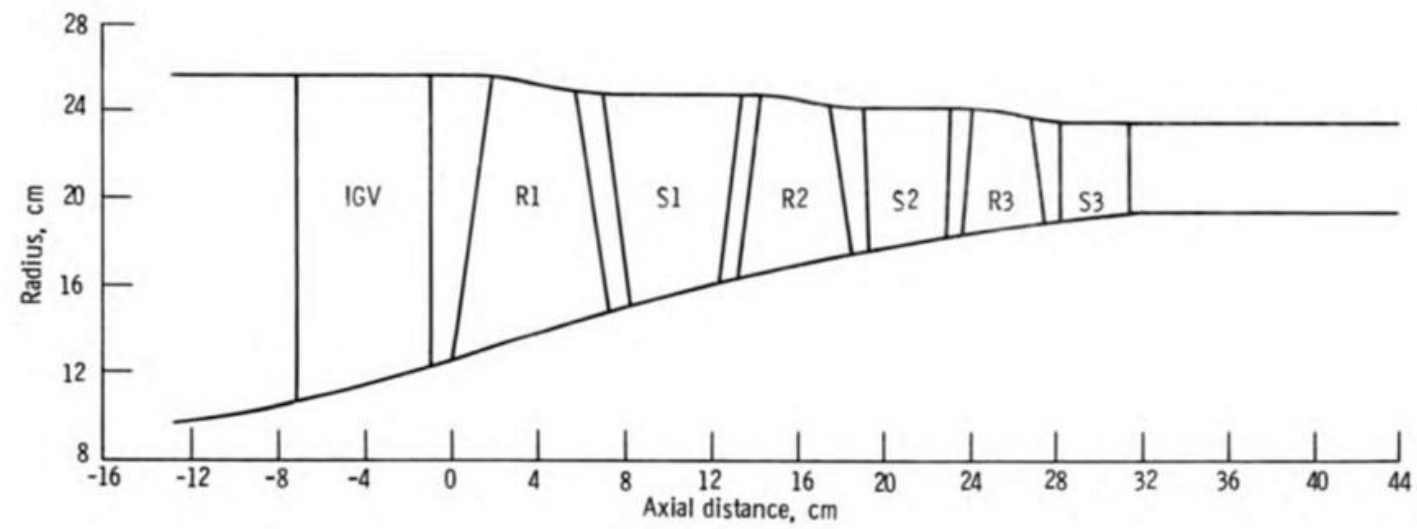

Figure 4.14: Geometry for NASA 74A [27]

Table 4.8: Efficiency Distribution for NASA 74A

\begin{tabular}{|c|c|c|c|}
\hline Annulus & \multicolumn{3}{|c|}{ Efficiency } \\
\hline & Stage 1 & Stage 2 & Stage 3 \\
\hline Hub & 0.934 & 0.934 & 0.922 \\
\hline $\mathbf{2}$ & 0.931 & 0.931 & 0.923 \\
\hline $\mathbf{3}$ & 0.928 & 0.928 & 0.922 \\
\hline $\mathbf{4}$ & 0.920 & 0.920 & 0.920 \\
\hline $\mathbf{5}$ & 0.908 & 0.908 & 0.917 \\
\hline $\mathbf{6}$ & 0.896 & 0.896 & 0.914 \\
\hline $\mathbf{7}$ & 0.883 & 0.883 & 0.911 \\
\hline $\mathbf{8}$ & 0.870 & 0.870 & 0.908 \\
\hline $\mathbf{9}$ & 0.854 & 0.854 & 0.906 \\
\hline $\mathbf{1 0}$ & 0.833 & 0.833 & 0.900 \\
\hline $\mathbf{1 1}$ & 0.808 & 0.808 & 0.891 \\
\hline $\mathbf{1 2}$ & 0.795 & 0.795 & 0.885 \\
\hline Tip & 0.781 & 0.781 & 0.869 \\
\hline
\end{tabular}


Table 4.9: Input Parameters for NASA 74A

\begin{tabular}{|c|c|c|c|}
\hline \multicolumn{5}{|c|}{ NASA 74A } \\
\hline & $\begin{array}{c}\text { Hub } \\
(\mathrm{m})\end{array}$ & $\begin{array}{c}\text { Tip } \\
(\mathrm{m})\end{array}$ & $\begin{array}{c}\text { Number of } \\
\text { blades }\end{array}$ \\
\hline Ambient & 0.1051 & 0.2564 & \\
\hline IGV & 0.1051 & 0.2564 & 26 \\
\hline Rotor 1 & 0.1251 & 0.2561 & 28 \\
\hline Stator 1 & 0.1516 & 0.2485 & 34 \\
\hline Rotor 2 & 0.1649 & 0.2478 & 32 \\
\hline Stator 2 & 0.1771 & 0.2421 & 46 \\
\hline Rotor 3 & 0.1842 & 0.2418 & 39 \\
\hline Stator 3 & 0.1904 & 0.2368 & 54 \\
\hline \multicolumn{5}{|c|}{ Initial Parameters } \\
\hline Rotational Speed & 16042 & $\mathrm{rpm}$ \\
\hline \multicolumn{5}{|c|}{ Ambient Conditions } \\
\hline Ambient Pressure & 101400 & $\mathrm{rad} / \mathrm{s}$ \\
\hline Ambient Density & 1.09 & $\mathrm{~Pa}$ \\
\hline Mass Flow Rate & 29.71 & $\mathrm{~kg} / \mathrm{m}^{3}$ \\
\hline \multicolumn{5}{|c|}{ SLA Parameters } \\
\hline Number of Annuli & 13 & \multicolumn{1}{c}{} \\
\hline Number of Axial Stations & 8 & \\
\hline
\end{tabular}

Table 4.10: Exit Flow Angles for NASA 74A

\begin{tabular}{|c|c|c|c|}
\hline Annulus & \multicolumn{3}{|c|}{ Exit Flow Angles } \\
\hline & Rotor 1 & Rotor 2 & Rotor 3 \\
\hline Hub & 50.5 & 50.5 & 49.6 \\
\hline $\mathbf{2}$ & 49.9 & 49.5 & 48.9 \\
\hline $\mathbf{3}$ & 49.2 & 48.7 & 48.4 \\
\hline $\mathbf{4}$ & 48.0 & 47.4 & 47.6 \\
\hline $\mathbf{5}$ & 47.1 & 46.5 & 46.9 \\
\hline $\mathbf{6}$ & 46.2 & 45.7 & 46.4 \\
\hline $\mathbf{7}$ & 45.4 & 45.0 & 45.8 \\
\hline $\mathbf{8}$ & 44.6 & 44.3 & 45.2 \\
\hline $\mathbf{9}$ & 43.8 & 43.6 & 44.5 \\
\hline $\mathbf{1 0}$ & 43.4 & 43.4 & 44.2 \\
\hline $\mathbf{1 1}$ & 42.9 & 44.4 & 45.2 \\
\hline $\mathbf{1 2}$ & 42.6 & 45.2 & 45.9 \\
\hline Tip & 42.2 & 46.3 & 46.8 \\
\hline
\end{tabular}




\subsubsection{NASA 74A Results}

The output from CompFlow for NASA 74A are shown in the figures below. The axial velocity and Mach number distributions can be seen in Figure 4.15. One item to note is that the curvature of the profile becomes more pronounced with blade row along the compressor. Another item is that the Mach number of the last stator is less than the prior blade rows, which is actually a desired trait as the goal of the compressor is to slow the flow.

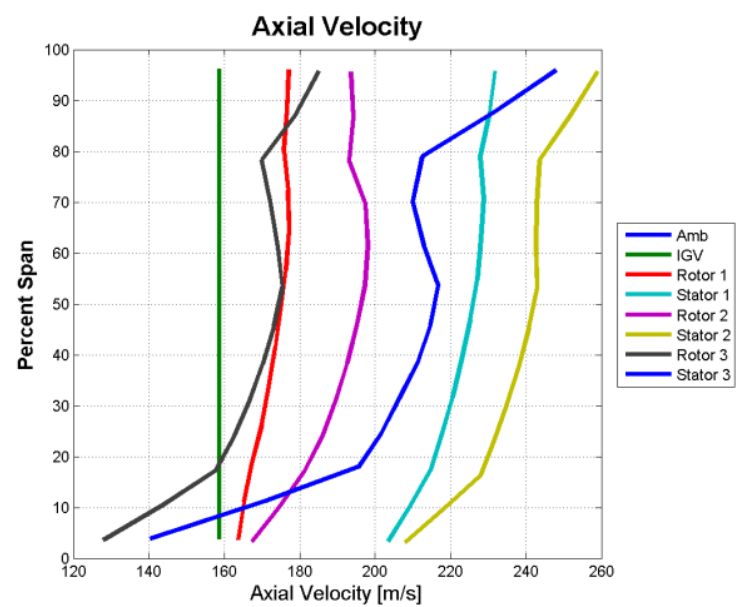

(a)

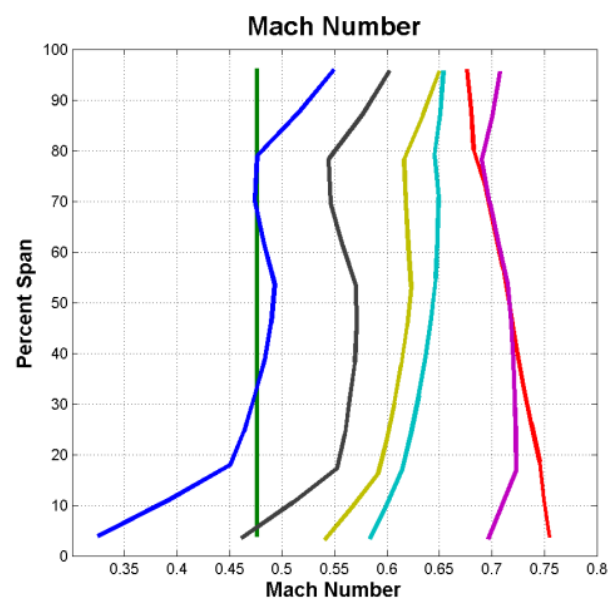

(b)

Figure 4.15:NASA 74A results for axial velocity (a) and Mach number (b)

Figure 4.16 shows the plots for total pressure ratio and total temperature ratio for each blade row. The results show that the total pressure ratio and total temperature ratio for all the stators is unity, which does accurately reflect the trend shown in Table 3.1. The pressure rise and temperature rise also increase with blade row, which intuitively makes sense for a compressor. 


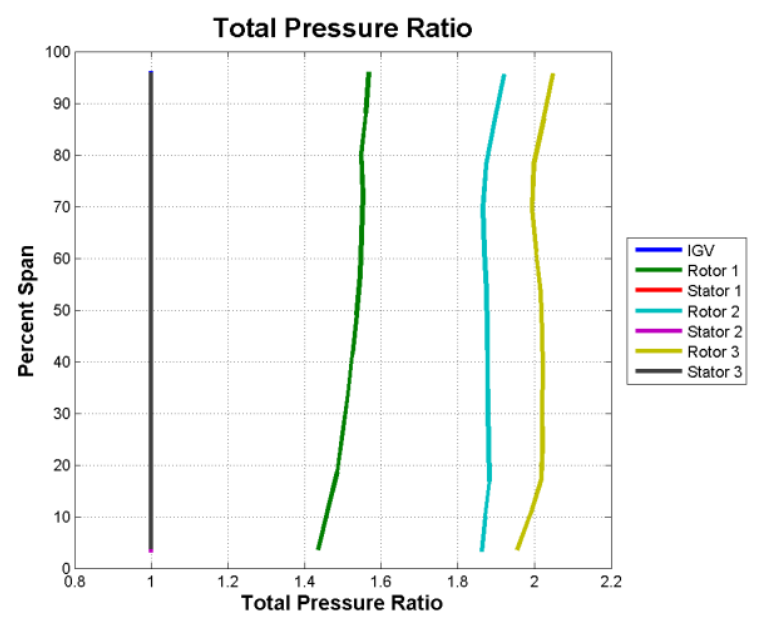

(a)

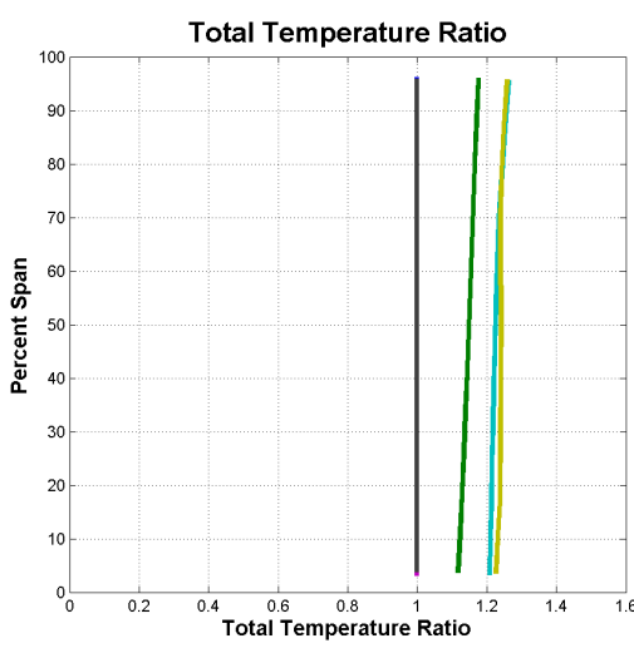

(b)

Figure 4.16: NASA 74A results for total pressure ratio (a) and total temperature ratio (b)

The total blade force and streamlines are plotted in Figure 4.17. Note that the streamlines follow the curvature as seen in Figure 4.14. The blade forces shown are closely grouped together, with the exception of Rotor 3.

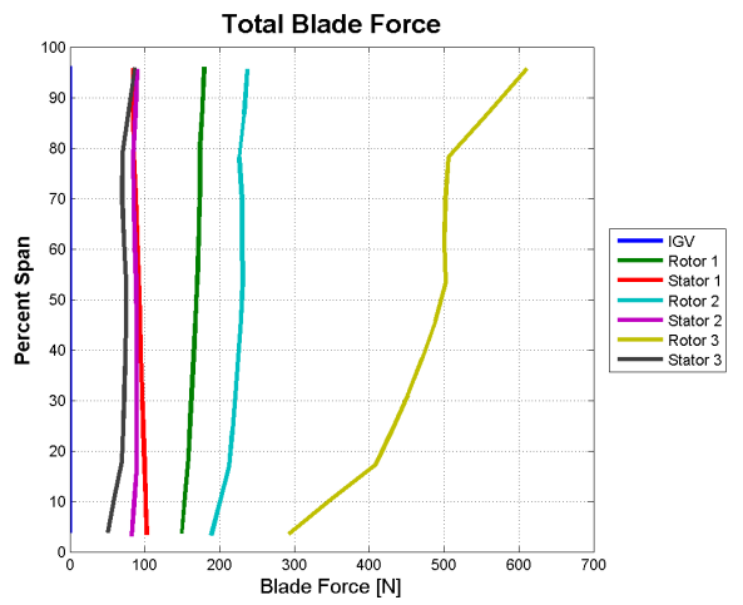

(a)

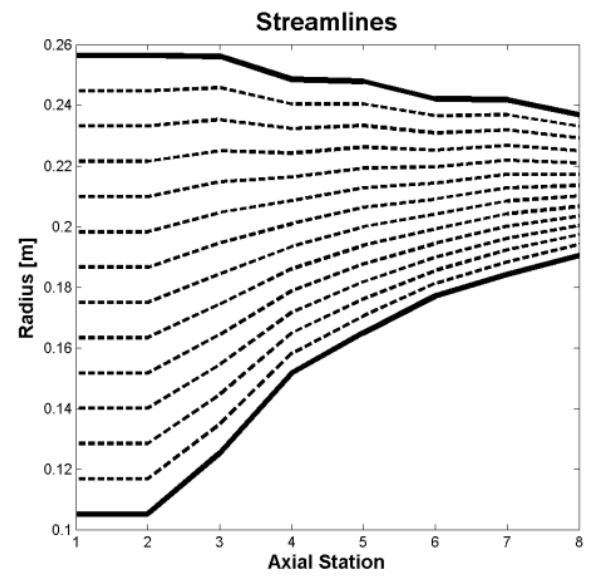

(b)

Figure 4.17: NASA 74A results for total blade force (a) and streamline (b)

Quantitative comparisons to experimental data can also be made for overall stage characteristics. Therefore, the values for total pressure ratio, the total temperature ratio, 
the adiabatic efficiency, and polytropic efficiency given by CompFlow for each stage were compared to experimental data provided by Steinke. Another comparison point for this data set is with values extracted from a mean-line code. Veres also tests NASA 74A using a mean-line code developed at NASA called COMDES [10]. This code serves as a rapid assessment tool for preliminary design phase of compressors. The values calculated by COMDES for NASA 74A are compared against both CompFlow data and experimental values provided by Steinke, as shown in Table 4.11.

Table 4.11: Comparison of computational and experimental data for NASA74A

\begin{tabular}{|l|c|c|c|c|}
\hline & $\begin{array}{c}\text { Total Pressure } \\
\text { Ratio }\end{array}$ & $\begin{array}{c}\text { Total Temperature } \\
\text { Ratio }\end{array}$ & $\begin{array}{c}\text { Adiabatic } \\
\text { Efficiency }\end{array}$ & $\begin{array}{c}\text { Polytropic } \\
\text { Efficiency }\end{array}$ \\
\hline CompFlow & 1.521 & 1.148 & 0.852 & 0.861 \\
\hline Steinke Data & 1.743 & 1.209 & 0.823 & 0.836 \\
\hline COMDES & 1.658 & 1.189 & 0.825 & \\
\hline & \multicolumn{5}{|c|}{ Stage 2 } \\
\hline CompFlow & 1.880 & 1.228 & 0.857 & 0.869 \\
\hline Steinke Data & 1.691 & 1.181 & 0.849 & 0.859 \\
\hline COMDES & 1.645 & 1.180 & 0.852 & \\
\hline & \multicolumn{5}{|c|}{ Stage 3 } \\
\hline CompFlow & 2.010 & 1.241 & 0.906 & 0.915 \\
\hline Steinke Data & 1.613 & 1.159 & 0.863 & 0.872 \\
\hline COMDES & 1.579 & 1.162 & 0.863 & \\
\hline
\end{tabular}

The above results show that the pressure rise determined by CompFlow increases across stage rows, whereas the experimental data and COMDES show the opposite trend. Therefore, CompFlow is over predicting the stage performance for NASA 74A. This result is puzzling as CompFlow did well for matching a single stage using Stage 37. Another case was tested using the same geometry but dropping the ambient axial velocity to $140 \mathrm{~m} / \mathrm{s}$, when it was originally predicted to be $158.6 \mathrm{~ms} / \mathrm{s}$, in an attempt to force a lower pressure rise. The results of this study are more promising and show a better agreement between parameters from CompFlow and experimental data. 
Table 4.12: NASA 74A results for ambient velocity $=140 \mathrm{~m} / \mathrm{s}$

\begin{tabular}{|l|c|c|}
\hline & $\begin{array}{c}\text { Total Pressure } \\
\text { Ratio }\end{array}$ & $\begin{array}{c}\text { Total Temperature } \\
\text { Ratio }\end{array}$ \\
\hline CompFlow & \multicolumn{3}{|c|}{ Stage 1 } \\
\hline Steinke Data & 1.522 & 1.148 \\
\hline & 1.743 & 1.209 \\
\hline CompFlow & 1.732 & Stage 2 \\
\hline Steinke Data & 1.691 & 1.196 \\
\hline & \multicolumn{3}{|c|}{ Stage 3 } \\
\hline CompFlow & 1.934 & 1.181 \\
\hline Steinke Data & 1.613 & 1.227 \\
\hline
\end{tabular}

\subsection{Discussion of Code Verification and Validation}

The purpose of this chapter was to conduct a verification and validation study on CompFLow. Verification primarily involved debugging the code and ensuring that the flow of calculations was correct. This study was performed by utilizing a single-stage compressor with known outputs as provided by Flack [5]. Additionally, sensitivity studies on the mesh size for the computational domain were performed. The results of this study yield the recommended number of annuli to be between 9 and 15 . An offdesign study was also performed in which the rotational speed was decreased by increments. As the matching of the computational data with the experimental data was poor for this study, CompFlow is not currently recommended for use in off-design studies.

Once the code was verified to be working correcting, validation studies were conducted in which the results from CompFlow were compared to experimental data. Results from CompFlow compared well against experimental data for single-stage 
geometries, as seen with Stage 37. However, multi-stage compressors had a more difficult time matching with experimental values. Decreasing the ambient axial velocity appeared to help with matching. However, caution should be used for decreasing the velocity to ensure that the updated value is still meaningful for the flow physics. The next chapter will attempt a case study on two connected multi-stage compressors, with the geometry provided by GE Aviation. 


\section{Chapter 5}

\section{GEnx-2B Case Study}

\subsection{Development of GEnx-2B}

Within recent years, there has been an increased consciousness on the impact of aviation on the environment. NASA-driven standards and more importantly economics have led all jet engine companies to begin developing quieter, cleaner and more fuel efficient products. General Electric (GE) Aviation has developed a line of next generation engines, called GEnx. The GEnx family of engines was developed to power Boeing's 787 Dreamliner and the new Airbus A350 [28]. It will reduce harmful emissions to almost 95\% below today's standards. This chapter will conduct a study on the GEnx-2B engine utilizing CompFlow. The goal of this study is to match the overall pressure ratio as rated by GE.

\subsection{Geometry of GEnx-2B}

The GEnx-2B engine is comprised of several innovative technologies. This engine achieves the highest pressure ratio in aviation while using a low number of high-pressure compressor stages. It also uses an innovative manufacturing technique of bladed disks, referred to as "blisks". This single bladed wheel contains fewer parts than a traditional blade and wedge wheel, therefore weighing less [3]. The GEnx-2B consists of a low- 
pressure compressor (LPC) and a high-pressure compressor (HPC). The LPC contains 3 stages, with an IGV at the inlet, and the HPC contains 10 stages. A schematic of this geometry is shown below in Figure 5.1, which was provided by GE. The dimensions for the hub and tip radii were obtained by scaling the below figure.

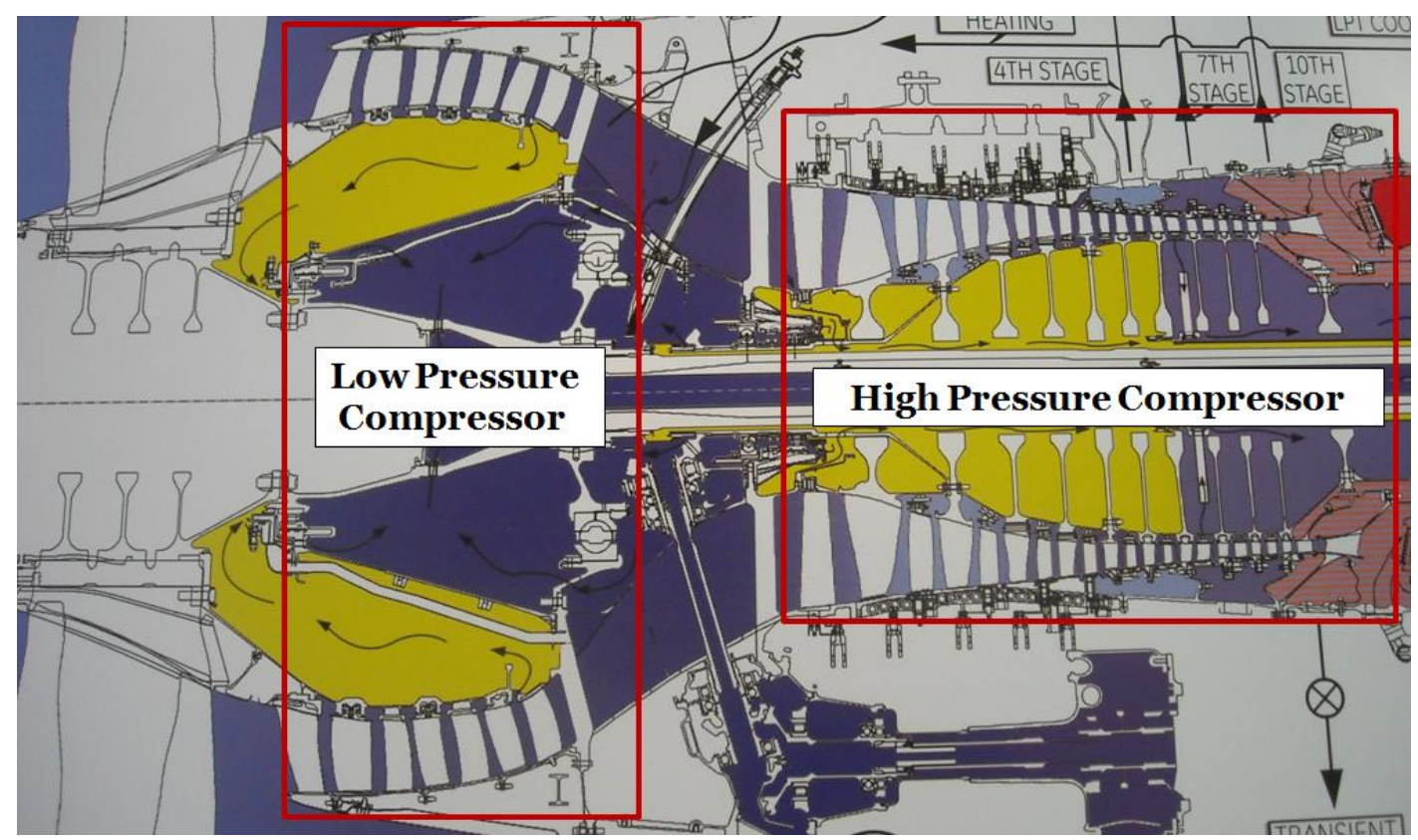

Figure 5.1: Schematic of GEnx-2B engine

Another item to note is that the nature of the LPC and HPC means the two shafts rotate at different speeds. At this point, CompFlow is not able to analyze two separately rotating shafts simultaneously for a compressor case. Therefore, for this study the LPC and HPC will need to be analyzed separately. The two cases will be connected by using the exit conditions of the LPC as the inlet conditions for the HPC. This method will allow for the proper flow of information between the two parts of the compressor. 


\subsubsection{Ambient Conditions}

A lot of the necessary geometric information to run this case was not available. Therefore, various assumptions and estimates have been made in order to obtain values. For this case, the ambient conditions were determined by assuming the engine is at an altitude of 35,000 ft. This altitude is representative for typical cruise conditions for most commercial airplanes. Table 5.1 summarizes the ambient data for this case. Note that this information is only valid for the LPC, as the exit conditions from the LPC will be the inlet conditions for the HPC.

Table 5.1: Ambient Conditions for GEnx-2B LPC

\begin{tabular}{|c|c|c|}
\hline \multicolumn{2}{|c|}{ Ambient Conditions } \\
\hline Altitude & 35,000 & $\mathrm{ft}$ \\
\hline Mach & 0.85 & \\
\hline Static Temperature & 394.1 & ${ }^{\circ} \mathrm{R}$ \\
\hline Static Pressure & 3.467 & $\mathrm{psia}$ \\
\hline Density & $7.382 \times 10^{-4}$ & $\mathrm{slug} / \mathrm{ft}^{3}$ \\
\hline Total Temperature & 452.4 & ${ }^{\circ} \mathrm{R}$ \\
\hline Total Pressure & 5.57 & $\mathrm{psia}$ \\
\hline
\end{tabular}

\subsubsection{GEnx-2B Geometry}

The geometry input for this case is summarized in the tables below. Table 5.2 describes the typical input parameters for CompFlow specific for this case. Note that the number of annuli is set to 9 , which is within the optimal range. Table 5.3 shows the hub and tip radii for the blade rows. For simplicity, the hub and tip radii were assumed to be constant for a blade row. The radial distribution of the efficiency is shown in Table 5.4. 
Again, the distribution of efficiency is assumed to be constant throughout the compressor for each blade row. A schematic of the LPC is shown in Figure 5.2.

Table 5.2: LPC Input Parameters for CompFlow

\begin{tabular}{|l|c|c|}
\hline \multicolumn{3}{|c|}{ GEnx-2B LPC } \\
\hline \multirow{2}{*}{ Rotational Speed } & 2835 & $\mathrm{rpm}$ \\
\cline { 2 - 3 } & 296.9 & $\mathrm{rad} / \mathrm{s}$ \\
\hline IGV Exit Angle & 15 & degrees \\
\hline Rotor Exit Angle & 50 & degrees \\
\hline Stator Exit Angle & 15 & degrees \\
\hline \multicolumn{3}{|c|}{ Ambient Conditions } \\
\hline Total Pressure & 5.57 & $\mathrm{psia}$ \\
\hline Total Temperature & 452.4 & ${ }^{\circ} \mathrm{R}$ \\
\hline Ambient Density & $7.38 \times 10^{-4}$ & $\mathrm{slug} / \mathrm{ft}^{3}$ \\
\hline Mass Flow Rate & 3.23 & $\mathrm{slug} / \mathrm{s}$ \\
\hline \multicolumn{3}{|c|}{ SLA Parameters } \\
\hline Number of Annuli & 9 & \\
\hline Number of Axial Stations & 8 & \\
\hline
\end{tabular}

Table 5.3: Radii Distributions for LPC

\begin{tabular}{|c|c|c|}
\hline \multicolumn{3}{|c|}{ GEnx-2B LPC Geometry } \\
\hline Stage Number & $\begin{array}{c}\text { Hub Radius } \\
\text { (ft) }\end{array}$ & $\begin{array}{c}\text { Tip Radius } \\
\text { (ft) }\end{array}$ \\
\hline IGV & 1.852 & 2.260 \\
\hline 1 & 1.852 & 2.260 \\
\hline 2 & 1.852 & 2.202 \\
\hline 3 & 1.808 & 2.129 \\
\hline
\end{tabular}

Table 5.4: Efficiency Distribution for LPC

\begin{tabular}{|c|c|}
\hline \multicolumn{2}{|c|}{ GEnx-2B LPC } \\
\hline $\begin{array}{c}\text { Annulus } \\
\text { Number }\end{array}$ & Efficiency \\
\hline Hub & 0.920 \\
\hline $\mathbf{2}$ & 0.911 \\
\hline $\mathbf{3}$ & 0.903 \\
\hline $\mathbf{4}$ & 0.894 \\
\hline $\mathbf{5}$ & 0.885 \\
\hline $\mathbf{6}$ & 0.876 \\
\hline $\mathbf{7}$ & 0.868 \\
\hline $\mathbf{8}$ & 0.859 \\
\hline Tip & 0.850 \\
\hline
\end{tabular}




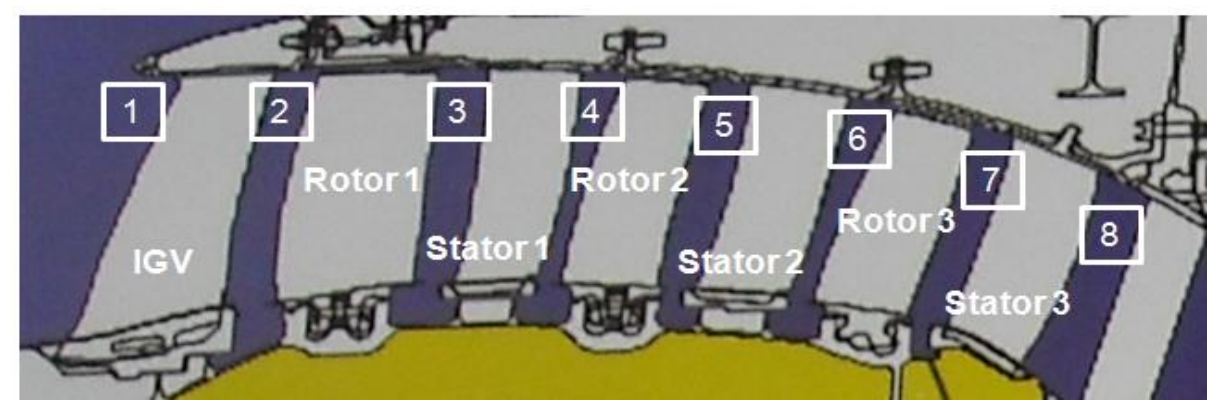

Figure 5.2: Schematic of GEnx-2B LPC [29]

\subsubsection{Theoretical Results}

Before proceeding with calculations, it was necessary to obtain "theoretical" values for comparison with the results as experimental data is not available. These calculations were done by assuming a constant temperature rise across all the stages. A typical value for the exit temperature of compressors is $1000^{\circ} \mathrm{F}$, which was used for this case. Knowing the inlet total temperature to be $452.4{ }^{\circ} \mathrm{R}$ from the above section, the temperature rise per stage was found to be $77.78{ }^{\circ} \mathrm{R}$. The total temperature ratio is then found for each stage by assuming a constant temperature rise across each stage. Knowing the inlet total pressure, the pressure for each stage can be calculated using Equation (3.30). The final total pressure using this method is found to be 213.93 psia, which yields a theoretical overall pressure ratio of 38.4. This is close to the overall pressure ratio of 43 as stated by the manufacturer, GE Aviation. 
Table 5.5: Input Parameters for Theoretical Calculations

\begin{tabular}{|l|c|c|}
\hline \multicolumn{3}{|c|}{ Theoretical Values } \\
\hline Inlet $\mathrm{Tt}$ & 452.4 & ${ }^{\circ} \mathrm{R}$ \\
\hline Exit $\mathrm{Tt}$ & 1000 & ${ }^{\circ} \mathrm{F}$ \\
\hline & 1459.7 & ${ }^{\circ} \mathrm{R}$ \\
\hline Number of Stages & 13 & \\
\hline$\Delta \mathrm{Tt}$ & 77.48 & ${ }^{\circ} \mathrm{R}$ \\
\hline Inlet $\mathrm{Pt}$ & 5.57 & $\mathrm{psia}$ \\
\hline Overall Pressure Ratio & 43 & \\
\hline
\end{tabular}

The theoretical values for total pressure and total temperature are plotted for each axial station in the figures below.

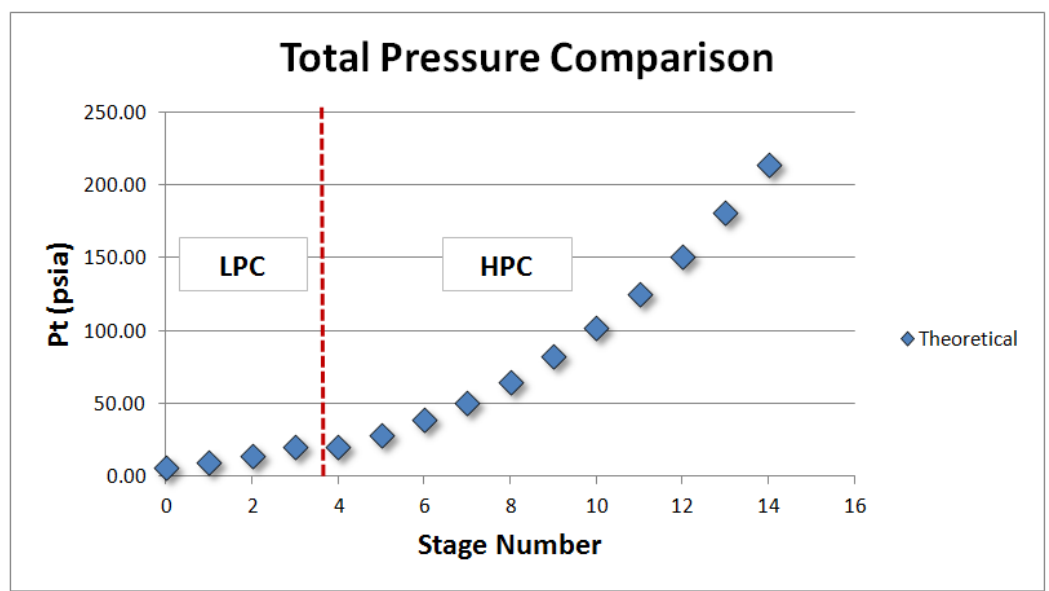

Figure 5.3: Theoretical total pressure values for GEnx-2b

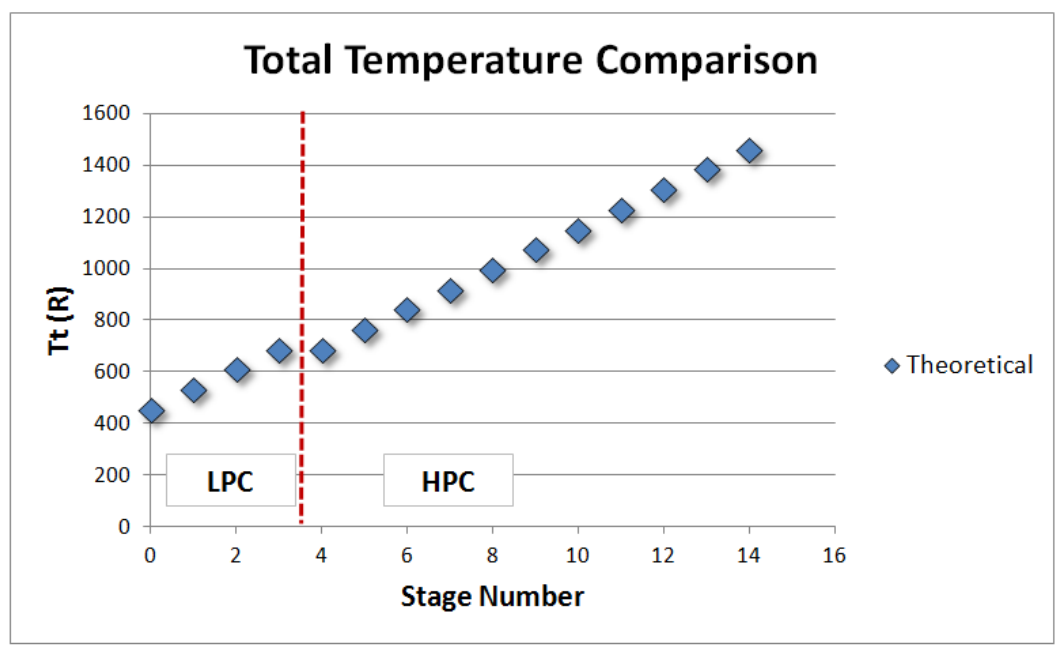

Figure 5.4: Theoretical values for total temperature for GEnx-2B 


\subsection{LPC Results}

The GEnx-2B LPC case was analyzed using the direct mode of calculations within CompFlow. The resulting plots are shown below. For this case, the number of blades per row was not known, so estimates were used based on industry standard. The values shown in Table 5.6 were chosen so that the blades would alternate periodically between rows. This implies that the blades from different stages would not match up as that would affect the flow conditions within the compressor. A combination of odd and even numbers will aid this process.

Table 5.6: Number of Blades Per Row in LPC

\begin{tabular}{|c|c|c|c|c|c|c|c|}
\hline Station & IGV & Rotor 1 & Stator 1 & Rotor 2 & Stator 2 & Rotor 3 & Stator 3 \\
\hline Blades & 58 & 62 & 64 & 63 & 65 & 62 & 60 \\
\hline
\end{tabular}
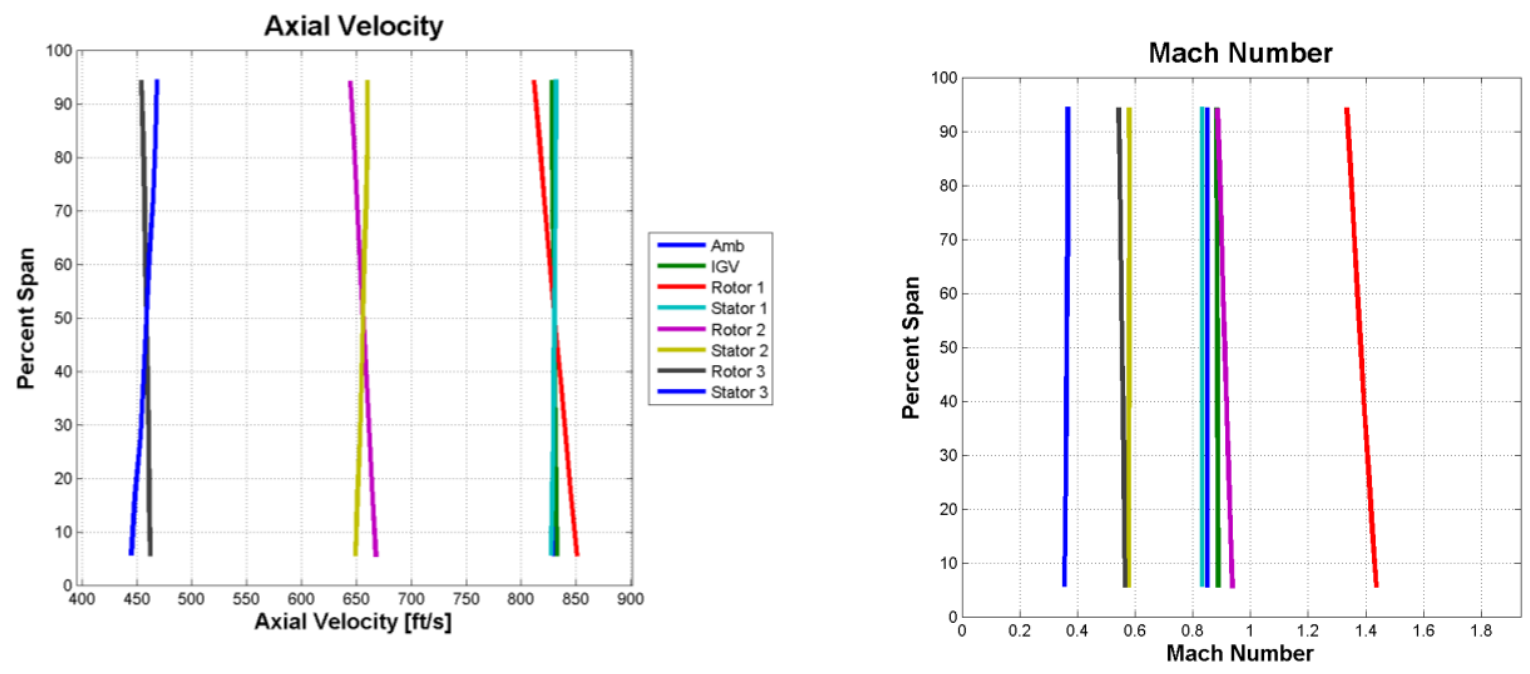

Figure 5.5: LPC distributions for (a) axial velocity and (b) Mach Number

The distribution for axial velocity above shows that the axial velocity decreased throughout the LPC. Looking at Figure 5.5a, it is clear that the axial velocities $\left(c_{a}\right)$ are 
grouped by stage, with the front stages to the rightmost portion of the graph and the exit stage towards the left side. Consulting Table 3.1 and examining the absolute velocity $(c)$ values, the LPC velocities do indeed follow the trend as described in the table. Considering Figure 5.5b, the Mach numbers are also grouped by stage. Another good indication is that the exit Mach number, denoted by the blue line for Stator 3, is between 0.35 and 0.40 which is typical for industry standards.

Figure 5.6 shows the estimates for the total blade force on each row. The plot shows that the maximum value is around $55 \mathrm{lbf}$, which occurs on Rotor 3 in the last stage. This value may seem large, but comparing it to the total thrust produced by the engine shows that it represents a small fraction of the thrust, which is between 53,000 and 75,000 lbf [3]. Figure 5.6b represents the streamline movement across the stages.
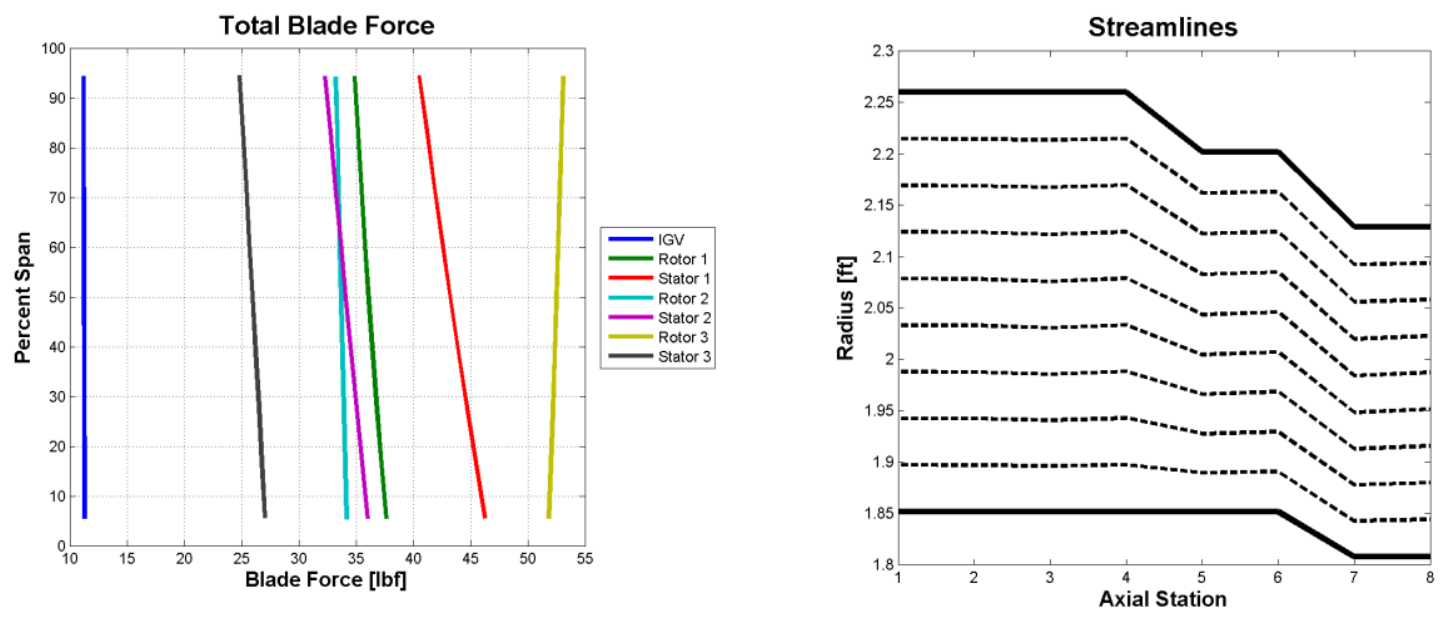

Figure 5.6: LPC results for (a) blade force distribution and (b) streamline movement

Instead of analyzing the pressure and temperature ratios across the stages, the actual values for total pressure and temperature will be compared against theoretical values. Figure 5.7 shows the comparison for the total pressures, where the theoretical values are plotted using blue diamonds, and CompFlow values using red dots. The figure 
shows that there is good agreement between the two sets of data, except near the end. However, the difference between these values is still less than $10 \%$, therefore the agreement is deemed reasonable. The total temperature comparison seen in Figure 5.7 follows the same trend as the total pressure values.

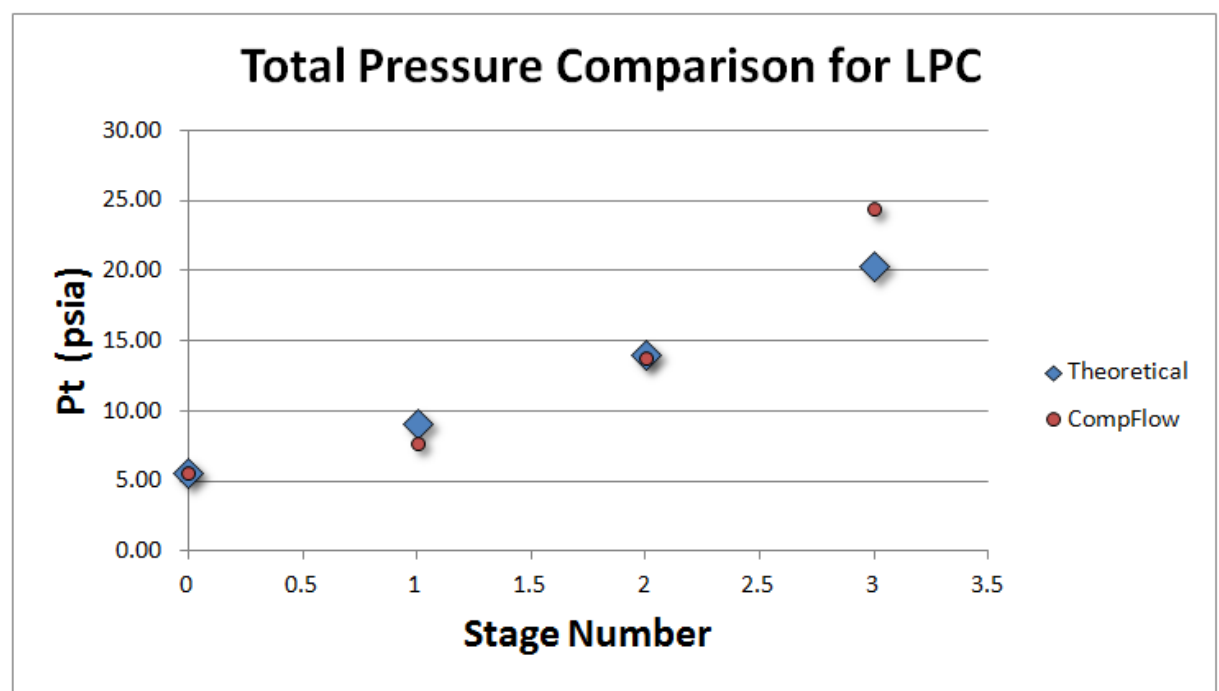

Figure 5.7: Comparison of theoretical total pressures to CompFlow results

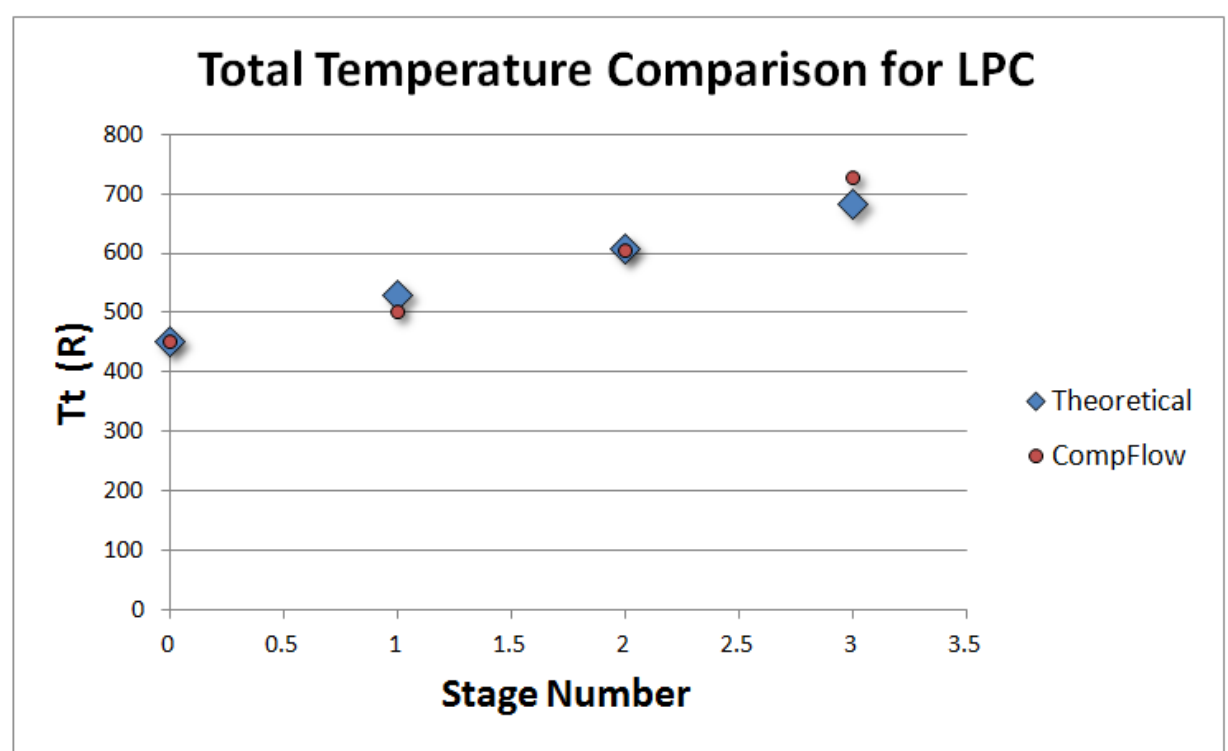

Figure 5.8: Comparison of theoretical total temperatures to CompFlow results 


\subsection{GEnx-2B HPC}

The high pressure compressor consists of an inlet guide vane and ten stages of rotors and stators, as shown in Figure 5.9 . Table 5.7 provides the hub and tip radii for each stage. The input parameters used for this case are listed in Table 5.8. The efficiency behind each blade row is evenly distributed in the radial direction from 0.92 at the hub to 0.85 at the tip. The exit flow angles are kept constant across the blade span for each blade row. Note that the ambient conditions for the HPC case seen in the table are taken from the exit conditions for the LPC.

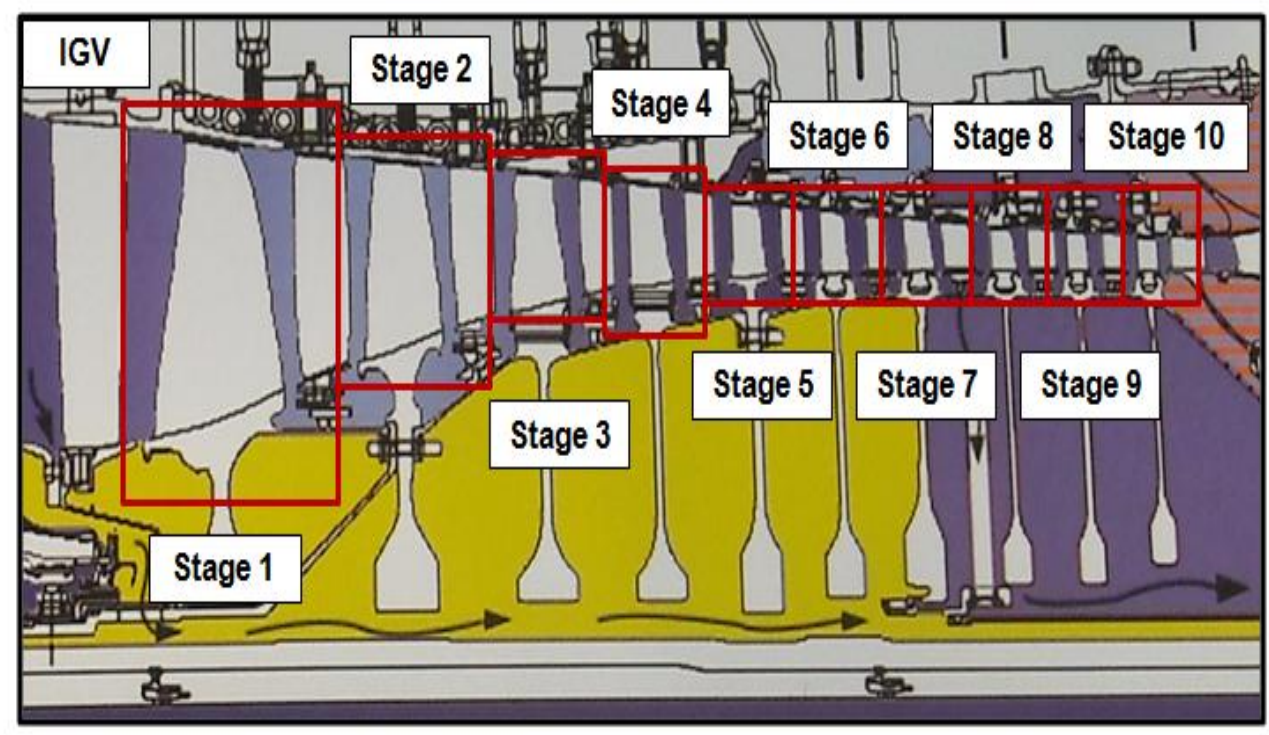

Figure 5.9: Schematic of GEnx-2B HPC [29]

Table 5.7: Radii Distribution for HPC

\begin{tabular}{|c|c|c|c|c|c|c|c|c|c|c|c|}
\hline Stage & IGV & $\mathbf{1}$ & $\mathbf{2}$ & $\mathbf{3}$ & $\mathbf{4}$ & $\mathbf{5}$ & $\mathbf{6}$ & $\mathbf{7}$ & $\mathbf{8}$ & $\mathbf{9}$ & $\mathbf{1 0}$ \\
\hline $\begin{array}{c}\text { Hub Radius } \\
\text { (ft) }\end{array}$ & 0.583 & 0.656 & 0.620 & 0.802 & 0.839 & 0.875 & 0.875 & 0.875 & 0.875 & 0.875 & 0.875 \\
\hline $\begin{array}{c}\text { Tip Radius } \\
\text { (ft) }\end{array}$ & 1.276 & 1.203 & 1.167 & 1.131 & 1.094 & 1.094 & 1.058 & 1.058 & 1.058 & 1.021 & 1.021 \\
\hline
\end{tabular}


Table 5.8: HPC Input Parameters for CompFlow

\begin{tabular}{|c|c|c|}
\hline \multicolumn{2}{|c|}{ GEnx-2B HPC } & Initial Parameters \\
\hline \multirow{2}{*}{ Rotational Speed } & 11377 & rpm \\
\hline & 1191.4 & $\mathrm{rad} / \mathrm{s}$ \\
\hline Efficiency & $\begin{array}{l}0.92 \\
0.85 \\
\end{array}$ & $\begin{array}{l}\text { hub } \\
\text { tip }\end{array}$ \\
\hline IGV Exit Angle & 10 & degrees \\
\hline Rotor Exit Angle & 45 & degrees \\
\hline Stator Exit Angle & 10 & degrees \\
\hline \multicolumn{3}{|c|}{ Ambient Conditions } \\
\hline Total Pressure & 24.45 & psia \\
\hline Total Temperature & 472.9 & ${ }^{\circ} \mathrm{R}$ \\
\hline Ambient Density & $1.77 \times 10^{-3}$ & $\operatorname{slug} / \mathrm{ft}^{3}$ \\
\hline Mass Flow Rate & 3.23 & slug/s \\
\hline \multicolumn{3}{|c|}{ SLA Parameters } \\
\hline Number of Annuli & 9 & \\
\hline Number of Axial Stations & 22 & \\
\hline
\end{tabular}

\subsubsection{HPC Results}

At this time, CompFlow was not able to converge on a solution for the HPC case. The calculations are possible only up to Rotor 2 in the second stage, at which point any estimate for axial velocity entered within a valid range would not lead to convergence. This could be affected by the numerous assumptions made to this point for the geometry. A jet engine axial compressor is an environment with severe adverse pressure gradients, and the performance is very sensitive to the compressor geometry. As mentioned earlier, commercial commercials often contain variable stator geometries. As the exit angles were estimated for this case study, along with the ambient conditions, the values chosen most 
likely do not represent optimal values for the given operating conditions. Changing the exit flow angles may affect the convergence of this case study. Future work should include a more detailed analysis of compressors with a high number of stages. 


\section{Chapter 6}

\section{Conclusions}

\subsection{Accomplishments}

The study and analysis of axial compressors is an integral step in the production of jet engines. The examination of vibratory effects due to running these high-speed compressors is crucial for the design phase. The work presented in this thesis provides a tool for estimating the force and moment components that will be used for rotordynamic calculations.

The major product of this thesis is packaged as an "aerodynamic module" for use within ROMAC's software RotorSol. A GUI was developed to provide users with the means of conducting an aerodynamic analysis for axial compressors, labeled as CompFlow. Instructions for running this software have been provided in Appendix C. The governing model of CompFlow is based on the "streamline curvature" method as devised by $\mathrm{Wu}$ and later modified by Flack. One major advantage of CompFlow is that the run-time is much less than the computational time and power necessary for full CFD calculations. A verification and validation study was conducted on CompFlow to determine the accuracy of the solutions presented.

The verification study conducted on the code showed that the results from CompFlow yielded similar results as to a simple compressor case provided by Flack. This process primarily involved debugging the code for errors. The validation study involved comparing results from CompFlow to compressor cases with experimental data available. 
This study was conducted using open sources cases from NASA called Stage 37 and NASA 74A. The results from CompFlow had excellent agreement with Stage 37 experimental data. However, for NASA 74A the values from CompFlow were found to overestimate the experimental values.

Sensitivity studies were also conducting using the validation cases. One such study analyzed the effect of varying the annuli number on accuracy. This result yielded an optimal annuli range of 9 to 15 . Another study conducted studied decreasing the rotational speed of Stage 37 in CompFlow as an "off-design" analysis. The study showed that there was increasingly poor matching of data with experimental values as the rotational speed was decreased.

Finally, a case study was conducted on an actual commercial jet engine, the GEnx2B. This engine was developed by GE Aviation as a next generation product that operates more quietly and cleanly. As experimental data was not available, theoretical values were calculated based on assumptions about the operating condition. This compressor case is divided into two portions, the low-pressure compressor and the high-pressure compressor. CompFlow was able to run the LPC case and obtain reasonable agreement with the theoretical values. The code was not able to process the HPC case for the GEnx$2 \mathrm{~b}$, which is most likely due to the numerous assumptions made for the geometry.

\subsection{Future Work}

As the majority of this thesis was devoted to developing software, numerous improvements can be expected to the code. One item that will need to be addressed is the 
integration of CompFlow with RotorSol. This process will involve ensuring that data flows between the two software modules correctly and debugging any errors associated with integration. This step may also include updating the current GUI to a new version and to match the look of RotorSol.

Another project component could involve obtaining more compressor cases with available experimental data where the input and output parameters are detailed. Such experimental cases could be provided by Penn State as well as NASA. As validation was an important component of the thesis work, additional studies would help to increase the confidence in the accuracy of the code. Within this thesis, the results from CompFlow were compared to mean-line values. However, running full CFD cases for comparison would add another valuable study towards validation.

Although CompFlow is able to model some compressible effects, it does not fully account for end-wall losses or viscous effects. Adding boundary layer analysis to the code would help to account for viscous effects near the end-wall regions of the compressor. Another component to add to the code would be loss-modeling. This is partially accounted for by the efficiency distribution, but a more detailed analysis would help to define the effects near the end-wall region.

Finally, integrated use of CompFlow within industry could be another project. As this code was developed for use in rotordynamic calculations, input from the actual users would be crucial for further development. Beta testing of the code within the companies associated with ROMAC would allow for valuable feedback on the code's capabilities and areas for improvement. 


\section{Bibliography}

[1] Dixon, S., Fluid Mechanics and Thermodynamics of Turbomachinery, Pergamon Press Ltd., Boston, MA, 1998.

[2] Muszynska, A., Rotordynamics, CRC Press, Taylor \& Francis Group, Boca Raton, FL, 2005.

[3] "The GEnx Engine Family". [www.geaviation.com/engines/commericial/genx. Accessed 09/01/2011].

[4] Lakshminarayana, B., Fluid Dynamics and Heat Transfer of Turbomachinery, John Wiley \& Sons, Inc., New York, 1996.

[5] Flack, R., Fundamentals of Jet Propulsion with Applications, Cambridge University Press, Cambridge, UK, 2005.

[6] Gorla, R., and Khan, A., Turbomachinery: Design and Theory, Marcel Dekker, Inc., New York, 2003.

[7] De Choudhury, P., "Rotordynamic Stability Case Studies," International Journal of Rotating Machinery, Vol. 10, 2004.

[8] Adamczyk, J.J., “Aerodynamic Analysis of Multistage Turbomachinery Flows in Support of Aerodynamic Design," Journal of Turbomachinery, Vol. 122, 2000.

[9] Aungier, R., Axial-Flow Compressors: A Strategy for Aerodynamic Design and Analysis, ASME Press, New York, 2003.

[10] Veres, J., "Axial and Centrifugal Compressor Mean Line Flow Analysis Method," AIAA-2009-1641, Proc. 47th Aerospace Sciences Meeting, Orlando, FL, 2009.

[11] Wu, C., "A General Theory of Three-Dimensional Flow in Subsonic and Supersonic Turbomachines of Axial-, Radial- and Mixed-Flow Types”, NACA TN 1795, Washington, D.C., 1952.

[12] Denton, J.D., and Dawes, W.N., "Computational Fluid Dynamics for Turbomachinery Design," Proceedings of the Institute of Mechanical Engineers, Vol. 213, 1999.

[13] Lewis, R.I., Vortex Element Methods for Fluid Dynamics Analysis of Engineering Systems, Cambridge University Press, Cambridge, UK, 1991. 
[14] Dawes, W.N., "Turbomachinery Computational Fluid Dynamics: Asymptotes and Paradigm Shifts," Philosophical Transactions of the Royal Society, Vol. 365, 2007.

[15] Boretti, A., "Experimental and Computational Analysis of a Transonic Compressor Rotor," 17th Australasian Fluid Mechanics Conference, Auckland, New Zealand, 2010.

[16] Cumpsty, N., Compressor Aerodynamics, Longman Scientific and Technical, Essex, UK, 1989.

[17] Marsh, H., "A Digitial Computer Program for the Through-Flow Fluid Mechanics in an Arbitrary Turbomachine Using a Matrix Method," Aeronautical Research Council, 1966.

[18] Oates, G., Aerothermodynamics of Aircraft Engine Components, American Institute of Aeronautics and Astronautics, 1985.

[19] Horlock, J.H., and Denton, J.D., “A Review of Some Early Design Practice Using Computational Fluid Dynamics and a Current Perspective," Journal of Turbomachinery, Vol. 127, 2005.

[20] Anderson, J.D., Computational Fluid Dynamics: The Basics With Applications, McGraw-Hill, Inc., New York, 1995.

[21] Korpela, S.A., Principles of Turbomachinery, Wiley, Hoboken, NJ, 2011.

[22] Chaudhry, J.A., Sheth, P.N., and Allaire, P., "Matlab Rotor 2.0 Beta User Manual."

[23] Roy, C.J., "Review of Code and Solution Verification Procedures for Computational Simulation," Journal of Computational Physics, Vol. 205, 2005.

[24] Kleijnen, J.P.C, "Verification and Validation of Simulation Models," European Journal of Operational Research, Vol. 82, 1995.

[25] Denton, J., "Lessons from Rotor 37," Journal of Thermal Science, Vol. 6, No. 1, 1996.

[26] Reid, L., and Moore, R., "Performance of Single-Stage Axial-Flow Transonic Compressor with Rotor and Stator Aspect Ratios of 1.19 and 1.26, Respectively, and with design pressure ratio of 2.05," NASA TP 1659, 1980.

[27] Steinke, R., "Design of 9.271-Pressure-Ratio Five-Stage Core Compressor and Overall Performance for First Three Stages," NASA TP 2597, 1986. 
[28] "Driving GE Ecomagination with the Low-Emission GEnx Jet Engine," [http://www.geaviation.com/press/genx/genx_20050720.html. Accessed 02/10/2013].

[29] GE Aviation 


\section{Appendix A}

\section{Density Calculations}

In order to consider compressibility effects, CompFlow updates the density at the exit of each stage. This is done by determining the mean of the radial distributions of static pressure and temperature. These values are then used in the equation of state, see in Equation(3.11). The density is assumed to be constant along the radial direction for an axial location. The mean static temperature at the stage exit is then used to determine new values for $c_{p}$ and $\gamma$. Flack provides an equation that utilizes a least-squares fit of numeric data for the specific heat calculation [5].

$$
c_{p}=0.2269807 e^{0.000097247 \cdot T}
$$

The above equation outputs a value with English units of Btu/lbm- ${ }^{\circ} \mathrm{R}$, so the temperature will be converted to ${ }^{\circ} \mathrm{R}$ if it is not already so. To help reduce errors with unit conversions, all calculations for this process are done in English units, with the final answers being converted to metric if that is the system in use in the main script for CompFlow. The ratio for specific heats can be calculated using the following equation.

$$
\gamma=\frac{c_{p}}{c_{p}-R}
$$

The parameter $R$ seen above is the gas constant with the same units as $c_{p}$. As the end of the calculation the specific heat is converted to either $\mathrm{ft}^{2} /{ }^{\circ} \mathrm{R}-\mathrm{s}^{2}$ for English units or it is converted to $\mathrm{m}^{2} / \mathrm{K}-\mathrm{s}^{2}$ for metric units. 


\section{Appendix B}

\section{Summary of Equations}

$$
\begin{aligned}
& \text { Radial Equilibrium Momentum Equation } \\
& \begin{array}{c}
\frac{\partial V_{r}}{\partial t}+V_{r} \frac{\partial V_{r}}{\partial r}+V_{x} \frac{\partial V_{r}}{\partial x}-\frac{V_{u}{ }^{2}}{r}=-\frac{1}{\rho} \frac{\partial p}{\partial r}+F_{r} \\
\text { Radial Equilibrium Condition } \\
\frac{\partial p}{\partial r}=\rho \frac{V_{u}^{2}}{r} \\
(p+d p)(r+d r) d \theta-p \cdot r \cdot d \theta-2\left(p+\frac{d p}{2}\right) d r \frac{d \theta}{2}=\frac{d m}{2} c_{u}{ }^{2} \\
\text { Differential Pressure Analysis } \\
\text { Differential Mass } \\
d m=\rho \cdot r \cdot d \theta \cdot d r
\end{array}
\end{aligned}
$$

Free Vortex Distribution

$$
c_{u}=\frac{a}{r}
$$

Forced Vortex

$$
c_{u}=b r
$$

Half-Vortex

$$
c_{u}=\frac{a}{r}+b r
$$

Thermodynamic Expression for Entropy

$$
\frac{1}{\rho} \frac{d p}{d r}=\frac{d h}{d r}-T \frac{d s}{d r}
$$

Static Enthalpy

$$
h_{0}=h+\frac{V^{2}}{2}=h+\frac{V_{u}^{2}}{2}+\frac{V_{x}^{2}}{2}
$$


Modified Radial Equilibrium

$$
\frac{\partial p}{\partial r}=\rho\left(\frac{V_{u}^{2}}{r}+V_{u} \frac{\partial V_{u}}{\partial r}+V_{x} \frac{\partial V_{x}}{\partial r}\right)
$$

Ideal Equation of State

Radial Equilibrium with No Pressure Gradient

$$
p=\rho R T
$$

$$
V_{x} \frac{d V_{x}}{d r}+V_{u} \frac{d V_{u}}{d r}+\frac{V_{u}{ }^{2}}{r}=0
$$

Simplified Radial Equilibrium

$$
\frac{d V_{u}}{V_{u}}=-\frac{d r}{r}
$$

Discrete Simplified Radial Equilibrium

Average Radius

$$
\bar{R}_{i j}=\frac{1}{2}\left(R_{i j}+R_{i-1 j}\right)
$$

Ambient Axial Velocity Estimate

$$
c_{a}=\frac{\dot{m}}{\rho A}
$$

Inlet Area

$$
A=\pi\left(R_{T}{ }^{2}-R_{H}{ }^{2}\right)
$$

Axial Velocity

$$
c_{a_{i j}}=c_{i j} \cos \alpha_{i j}
$$

Tangential Velocity

$$
c_{u_{i j}}=c_{i j} \sin \alpha_{i j}
$$

Absolute Velocity

$$
c_{i j}^{2}=c_{a_{i j}}^{2}+c_{u_{i j}}^{2}
$$


Volumetric Flow Rate Using Even Spacing

$$
Q_{j}=\frac{\dot{m}}{\rho_{j} n}
$$

$$
\begin{gathered}
\text { Volumetric Flow Rate } \\
Q_{j}=c_{a_{i j}} \pi\left(R_{i+1 j}{ }^{2}-R_{i j}{ }^{2}\right)
\end{gathered}
$$

$$
\text { Blade Speed }
$$

$$
U_{i j}=\bar{R}_{i j} \omega
$$

Torque Equation (Euler Turbine)

$$
\begin{gathered}
T_{q_{i j}}=Q_{j}\left(\rho_{j+1} \bar{R}_{i j+1} c_{u_{i j+1}}-\rho_{j} \bar{R}_{i j} c_{u_{i j}}\right) \\
\text { Power } \\
\dot{W}_{i j}=T_{q_{i j}} \omega
\end{gathered}
$$

Relative Exit Flow Angle

$$
\begin{gathered}
\tan \beta_{i j}=\frac{c_{u_{i j}}-U_{i j}}{c_{a_{i j}}} \\
\text { Relative Velocity } \\
w_{i j}=\frac{c_{a_{i j}}}{\cos \beta_{i j}} \\
\text { Static Pressure } \\
p_{i j+1}=\rho_{j+1}\left[\dot{W}_{i j} \frac{\eta_{i j}}{\dot{m}_{a}}-\frac{1}{2}\left(c_{i j+1}{ }^{2}-c_{i j}{ }^{2}\right)-\frac{p_{i j}}{\rho_{j}}\right] \\
p_{t_{i j}}=\frac{\rho_{j}}{2} c_{i j}^{2}+p_{i j} \\
\text { Total Temperature } \\
\left\{\frac{1}{T_{t_{i j}}}\left[\left(\frac{p_{t_{i j}}}{p_{t_{i j-1}}}\right)^{\gamma / \gamma-1}-1\right]+1\right\}
\end{gathered}
$$


Static Temperature

$$
T_{s_{i j}}=T_{t_{i j}}-\frac{c_{i j}^{2}}{2 c_{p}}
$$

Speed of Sound

$$
a_{i j}=\sqrt{\gamma \cdot R \cdot T_{s_{i j}}}
$$

Mach Number

$$
M_{i j}=\frac{c_{i j}}{a_{i j}}
$$

Discrete Modified Radial Equilibrium

$$
\frac{\left(p_{i j}-p_{i-1 j}\right)}{\bar{R}_{i j}-\bar{R}_{i-1 j}}=\rho_{j i}\left[\frac{\bar{c}_{u_{i j}{ }^{2}}}{R_{i j}}+\bar{c}_{u_{i j}} \frac{\left(c_{u_{i j}}-c_{u_{i-1 j}}\right)}{\bar{R}_{i j}-\bar{R}_{i-1 j}}+\bar{c}_{a_{i j}} \frac{\left(c_{a_{i j}}-c_{a_{i-1 j}}\right)}{\bar{R}_{i j}-\bar{R}_{i-1 j}}\right]
$$

\section{Regula Falsi Method}

$$
x_{3}=\left[\frac{x_{1} y_{2}-x_{2} y_{1}}{y_{2}-y_{1}}\right]
$$

Total Pressure and Temperature Ratios

$$
P R_{j}=\frac{P t_{i j+1}}{P t_{i j}} \quad T R_{j}=\frac{T t_{i j+1}}{T t_{i j}}
$$

Overall Pressure Ratio

$$
\pi_{c}=\frac{P t_{s_{-} t o t}}{P t_{1}}
$$

Overall Temperature Ratio

$$
\tau_{c}=\pi_{c}^{\gamma-1 / \gamma}
$$

Adiabatic Efficiency

$$
\eta_{a d}=\frac{\pi_{c}{ }^{\gamma-1} / \gamma-1}{\tau_{c}-1}
$$

Polytropic Efficiency

$$
\eta_{p c}=\frac{\gamma-1}{\gamma} \frac{\ln \pi_{c}}{\ln \tau_{c}}
$$




\section{Percent Span}

$$
\% \text { span }=\frac{\bar{R}_{i j}-R_{h_{j}}}{R_{t_{j}}-R_{h_{j}}} \cdot 100
$$

Blade Spacing

$$
S_{j}=R_{h_{j}} \cdot \frac{2 \pi}{n}
$$

Blade Height

$$
h_{j}=R_{t_{j}}-R_{h_{j}}
$$

$$
\begin{gathered}
\text { Axial Force } \\
-F_{a_{i j}}=h_{j} \cdot S_{j}\left(p_{i j}-p_{i j-1}\right)+\rho_{j-1} \cdot c_{a_{i j-1}}{ }^{2} \cdot S_{j}\left(\frac{\rho_{j-1}}{\rho_{j}}-1\right)
\end{gathered}
$$

Tangential Force

$$
\begin{gathered}
F_{u_{i j}}=\rho_{j-1} \cdot h_{j} \cdot S_{j} \cdot c_{a_{i j-1}}\left(c_{u_{i j-1}}-c_{u_{i j}}\right) \\
\text { Total Force } \\
F_{i j}^{2}=F_{u_{i j}}^{2}+F_{a_{i j}}^{2}
\end{gathered}
$$

Tangential Moment

$$
M_{o_{j}}=F_{u m_{j}} \cdot R_{m_{j}}
$$

Mean Radius

$$
R_{m_{j}}=\frac{1}{2}\left(R_{t_{j}}-R_{h_{j}}\right)
$$

Specific Heat

$$
c_{p}=0.2269807 e^{0.000097247 \cdot T}
$$

\section{Ratio of Specific Heats}

$$
\gamma=\frac{c_{p}}{c_{p}-R}
$$




\section{Appendix C}

\section{CompFlow GUI User Guide}

This appendix will provide readers with the basics of navigating the CompFlow

GUI. Figure C.1 below displays the main window that is used for CompFlow. The steps to run CompFlow are described below. This GUI will be integrated into RotorSol to provide an "aerodynamic module" for compressors.

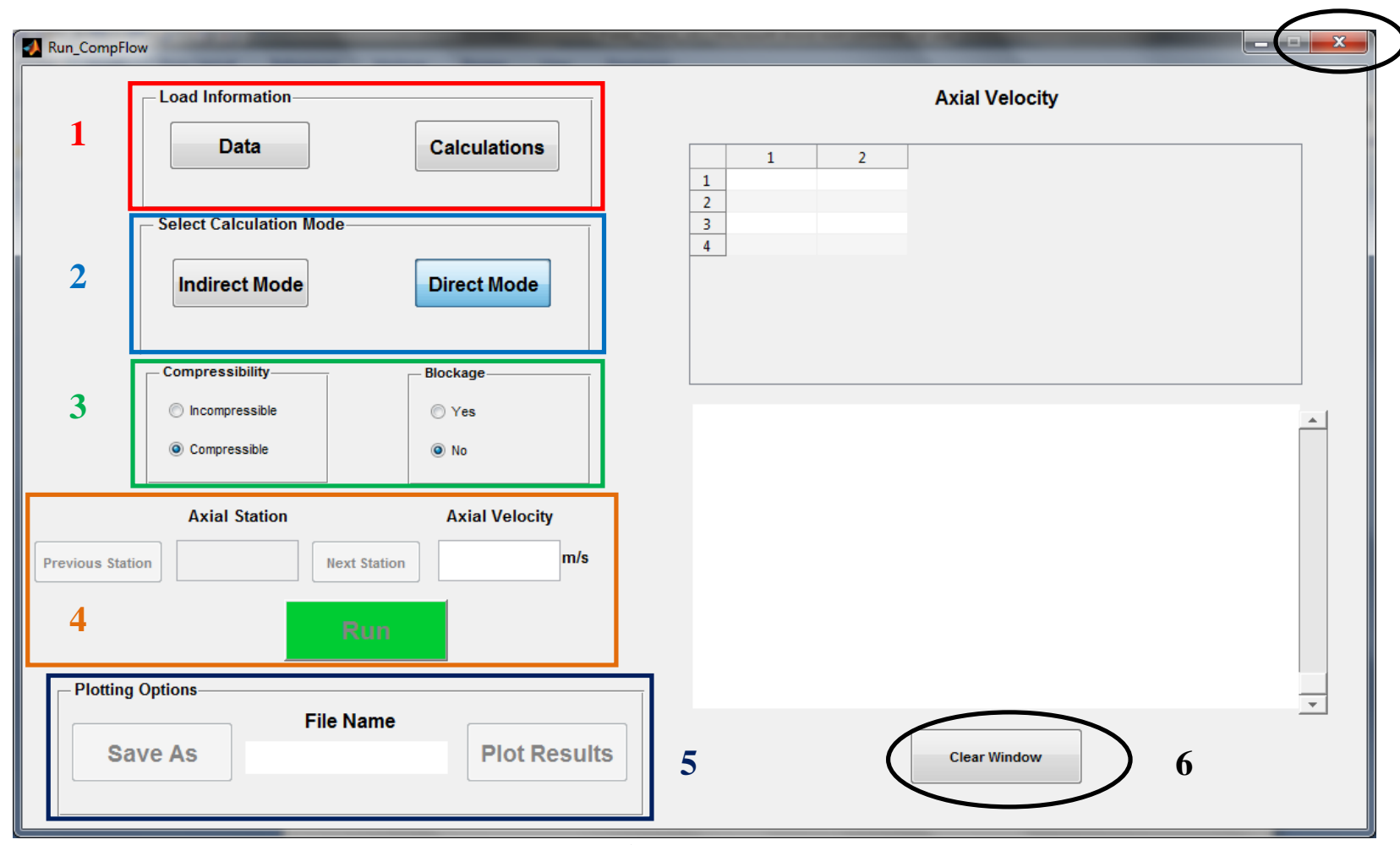

Figure C.1: CompFlow GUI main window 


\section{Step 1: Load Information}

The first step for running CompFlow is to load in the geometric data. There are two options for doing this, provided by the buttons labeled "Data" and "Calculations". These buttons are outlined in red seen in Figure C.1. If there is already data loaded into the GUI, a message box will warn the user that the data will be replaced upon a selection of a button.

\section{Data}

The "Data" option allows the user to enter geometric data manually into the GUI using the screen shown in Figure C.2. The flow of steps for manually entering the data is described below. It is recommended to manually enter the geometry for each new case or for new users. The GUI consists of a combination of text boxes, radio buttons and tables. For the text boxes and tables, be sure to hit "Enter" or "Tab" keys to lock in the data.

Table C.1: Steps for entering data into CompFlow

\begin{tabular}{|c|c|}
\hline Step & Action \\
\hline 1 & $\begin{array}{l}\text { - Enter numeric values for Number of Annuli and the total Number of Axial Stations } \\
\text { (include one for ambient conditions). } \\
\text { - The number of columns in Part } 5 \text { will update to reflect the axial stations. }\end{array}$ \\
\hline 2 & $\begin{array}{l}\text { - IGV: Select Yes if an inlet guide vane is in front of the first stage. Default is No } \\
\text { - Units: The units next to boxes in the GUI will update to reflect changes to this. }\end{array}$ \\
\hline 3 & $\begin{array}{l}\text { - Enter numeric values for Rotational Speed, Density and Mass Flow Rate. } \\
\text { - } \quad \text { GUI will calculate Ambient Axial Velocity and display it. } \\
\text { To change the value of the velocity, select Enter Velocity, and enter value in the box } \\
\text { above it. Note that a new density will be calculated. Selecting Enter Velocity again } \\
\text { will revert to the default settings. (Note: Enter original density again) }\end{array}$ \\
\hline 4 & $\begin{array}{l}\text { - Select either Static Pressure or Total Pressure for initialization. } \\
\text { - Enter corresponding values in the white text boxes }\end{array}$ \\
\hline 5 & $\begin{array}{l}\text { - Station Name: Enter the text name for each station. This will be used for the plot } \\
\text { legends } \\
\text { - Stage Number: Enter numeric values for the stage number each blade row }\end{array}$ \\
\hline
\end{tabular}




\begin{tabular}{|c|c|}
\hline & $\begin{array}{l}\text { corresponds to (e.g. "0" for ambient, "1" for IGV, etc.) } \\
\text { - Hub Radius: Enter numeric values for the hub radius of each blade row } \\
\text { - } \quad \text { Tip Radius: Enter numeric values for the tip (casing) radius of each blade row } \\
\text { - } \quad \text { Hit Done once the prior steps are complete to enable the options in Step } 6 .\end{array}$ \\
\hline 6 & $\begin{array}{l}\text { - The rows correspond to each blade row, and the columns to the number of annuli. If } \\
\text { the number of annuli or axial stations is changed, hit Done in Step } 5 \text { to update the } \\
\text { tables. } \\
\text { - Absolute Exit Flow Angle Distribution: Enter a value for each box to provide the } \\
\text { radial distribution of the exit flow angle (degrees) } \\
\text { - Exit Efficiency Distribution: Enter a value for each box to provide the radial } \\
\text { distribution of the efficiency. For IGV's and stators, enter the same efficiency } \\
\text { distribution as the rotor in that stage. Do not enter zeros. }\end{array}$ \\
\hline 7 & $\begin{array}{l}\text { - Import Data: Imports data saved to an Excel worksheet for a given case. For a new } \\
\text { case, manually enter the data first and then export it to ensure the proper formatting } \\
\text { of the Excel file. } \\
\text { - Export Data: Allows the user to save the data in the GUI to an Excel file to a folder } \\
\text { of their choice for later use. } \\
\text { - Load Data: All other sections should be complete before this step! Data from the } \\
\text { GUI will be saved and returned to the main window for computation. The data } \\
\text { window seen in Figure C. } 2 \text { will close. }\end{array}$ \\
\hline 8 & - This will close the window without saving any data. \\
\hline
\end{tabular}

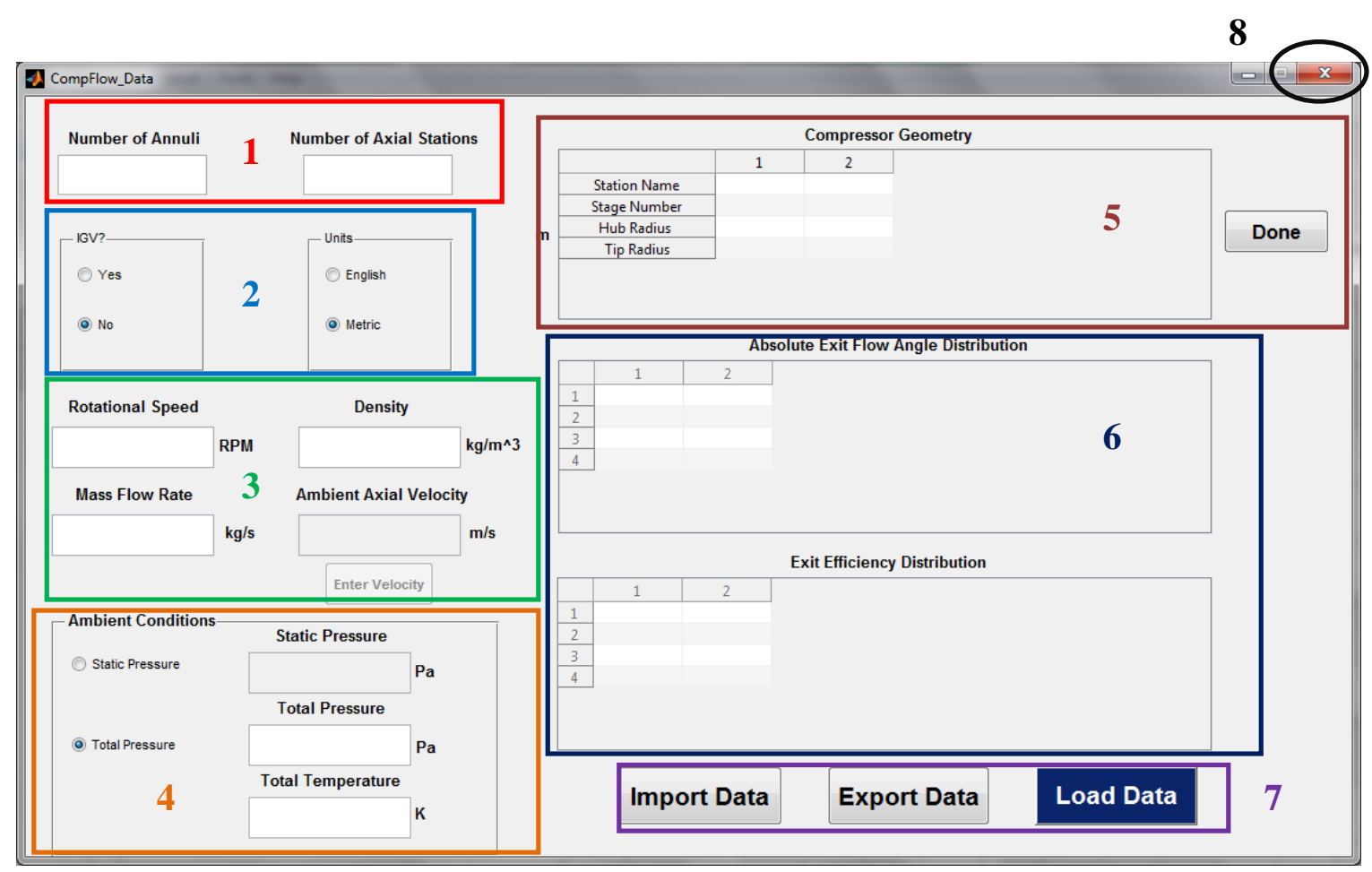

Figure C.2: GUI for "Data" button 


\section{Calculations}

The Calculations option allows a user to load data from a prior run, or data from an interupted case. The data for all cases are stored as a Matlab structure file (.mat). A another window will open that prompts the user to select a .mat file, shown in Figure C.3. If a user is attempting to load data from an interrupted case, the file "iter.mat" should be selected for the direct mode, and "vortex.mat" for the indirect mode.

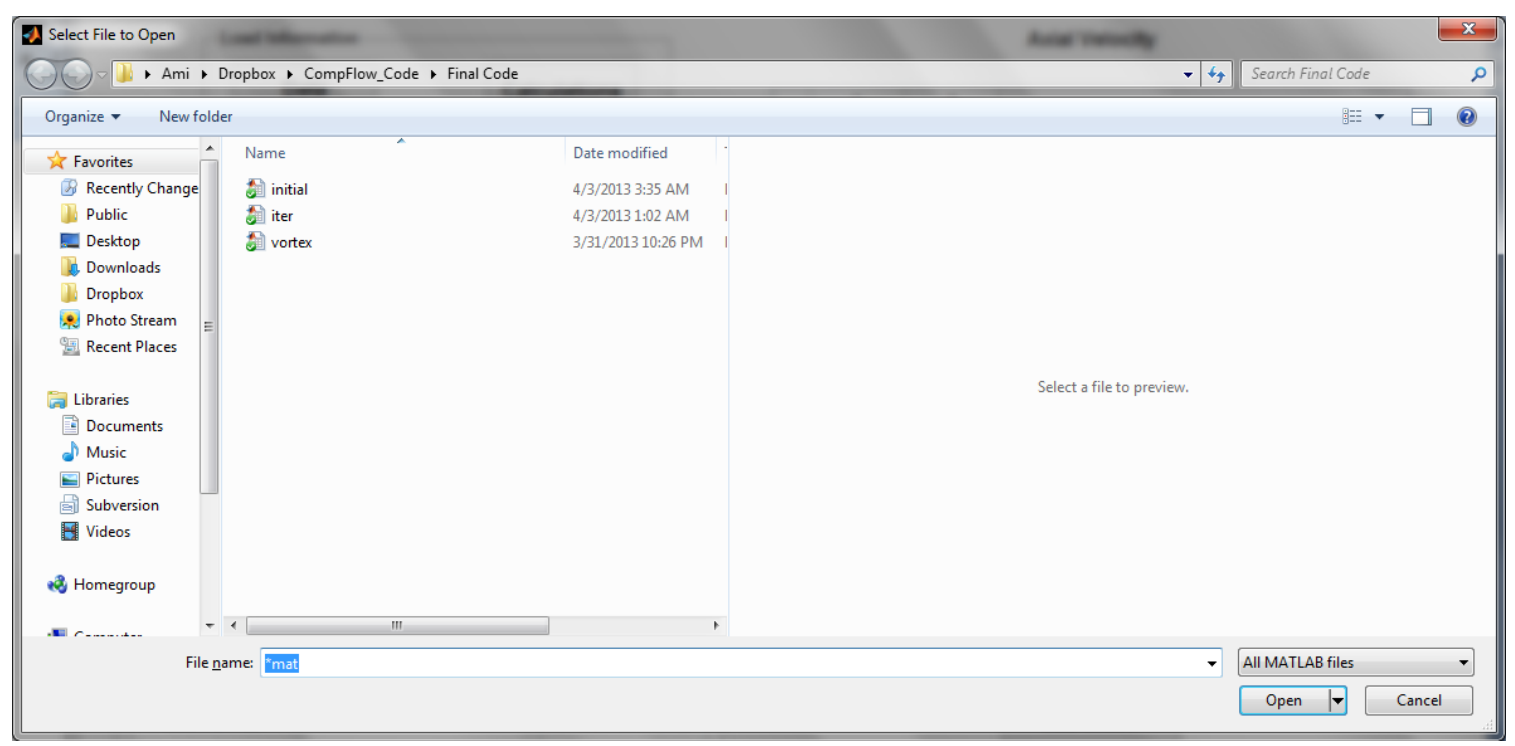

Figure C.3: Window for selecting .mat file using "Calculations" option in main window

Selecting Open will load the data into the main window and activate all the options, as seen below in Figure C.4. Hitting either Cancel or the red " $x$ " will return the user to the main window without loading any data. 


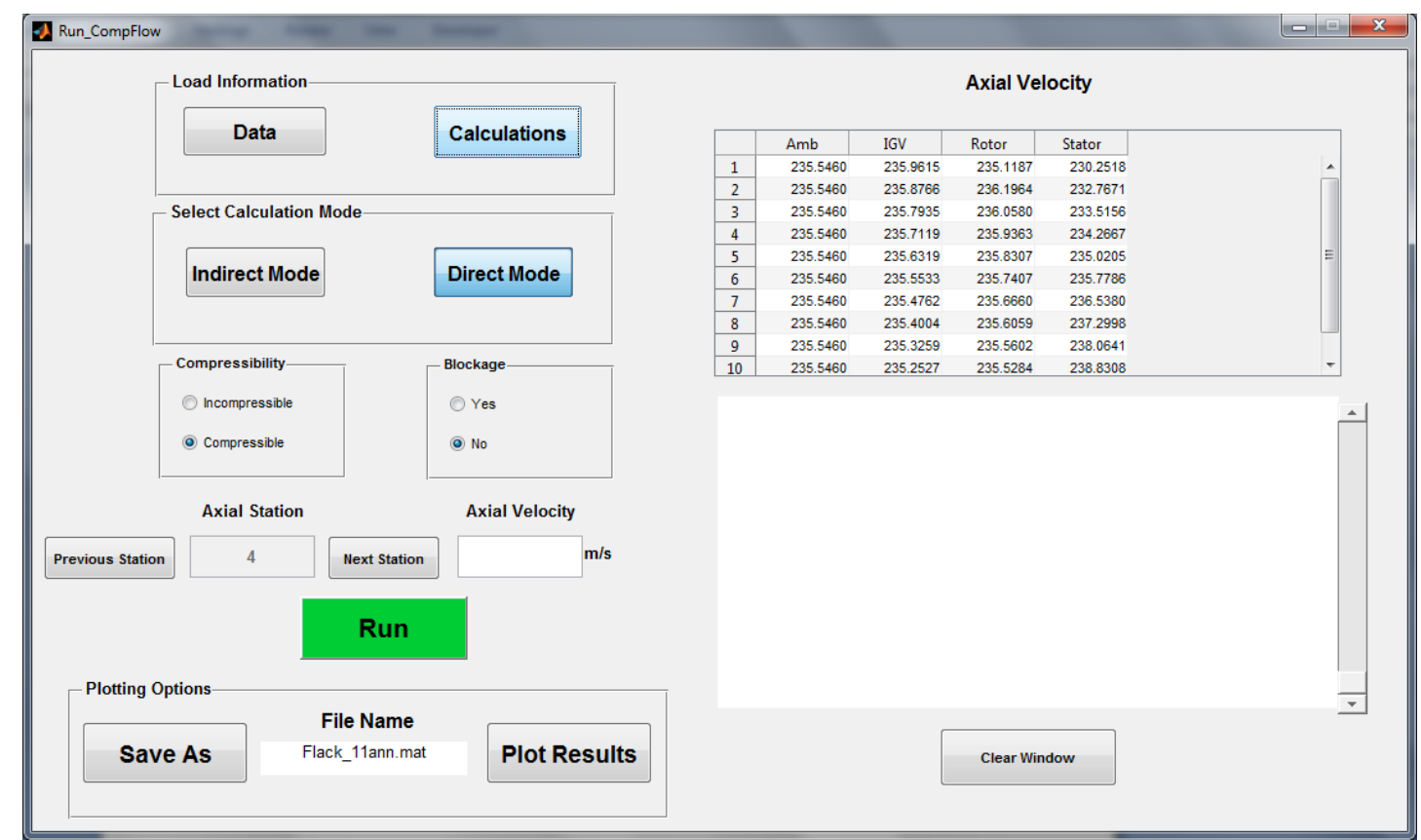

Figure C.4: Example of using "Calculations" option in main window

\section{Step 2: Select Calculation Mode}

Selecting Indirect Mode will allow a user to run a "free vortex" condition for the compressor being analyzed. This mode is very quick and does not require any iteration. The option Direct Mode will use an initial guess for the ambient velocity at the second station to iterate on the radial velocity profile for a given station. This mode will require user input at each axial station.

\section{Step 3: Compressibility and Blockage}

The user can decide whether to run an incompressible or compressible study by selecting the desired option. Incompressible will use the ambient density provided in 
Step 1 for all axial stations. Compressible will update the density at the exit of each stage. More information on the compressible calculations can be found in Appendix A.

\section{Step 4: Running Calculations}

When a new case is loaded, the Axial Station box in Figure C.1 is set to "2", so the calculations will start at the first axial station past ambient conditions. The table in the upper right corner will also show the velocity distribution for ambient conditions. To run calculations for an axial station, a velocity estimate should be entered in the Axial Velocity box. Hit Run and the iterations will show in the text box to the right with the slider. Once the calculations are done, the user will be able to increment to the next axial station using the Next Station button. If the iterations fail to converge, a message will appear in the text window, and a message box will appear with an error message. The user should then enter a new estimate for axial velocity and select Run again. Repeat this process until the iterations have converged for an axial station. Once convergence has been met for the last axial station, the options in Step 6 will be enabled.

\section{Step 6: Plotting Options}

\section{Save As}

After calculations have fully converged for a compressor case, the user will need to select a folder for saving the converged data. A window similar to the one seen in Figure C.3 will appear. The data will be stored as a Matlab structure (.mat) file. The folder the user selects will also be the location the plots are stored in. Once a location has 
been determined, the file name will appear in the text box and the Plot Results button will be activated.

\section{Plot Results}

Hitting this button will open another window, as seen below in Figure C.5. The table below describes the steps necessary to plot the results.

Table C.2: Steps for plotting results in CompFlow

\begin{tabular}{|c|ll|}
\hline Step & $\begin{array}{l}\text { Action } \\
\mathbf{1}\end{array}$ & $\begin{array}{l}\text { From the CompFlow main window, the name of the file from Step 6 Plotting Options } \\
\text { will be displayed here }\end{array}$ \\
$\begin{array}{c}\text { If the user desires to plot the results of a different case, the Load File button can be } \\
\text { used to load another .mat file. }\end{array}$ \\
\hline $\mathbf{2}$ & $\bullet \begin{array}{l}\text { The number of blades for each station needs to be entered here. Do not enter a value } \\
\text { of zero. }\end{array}$ \\
\hline $\mathbf{3}$ & $\begin{array}{l}\text { Selecting one of these options will plot the radial distribution of the parameter against } \\
\text { percent span in the plot in the center of the screen. The legend will contain the names } \\
\text { entered earlier in the process. }\end{array}$ \\
\hline $\mathbf{4}$ & $\begin{array}{l}\text { Save Current Figure will save the current plot in the middle of the window to the } \\
\text { directory chosen in Step 6 Plotting Options as a ".tif" file. }\end{array}$ \\
\hline
\end{tabular}

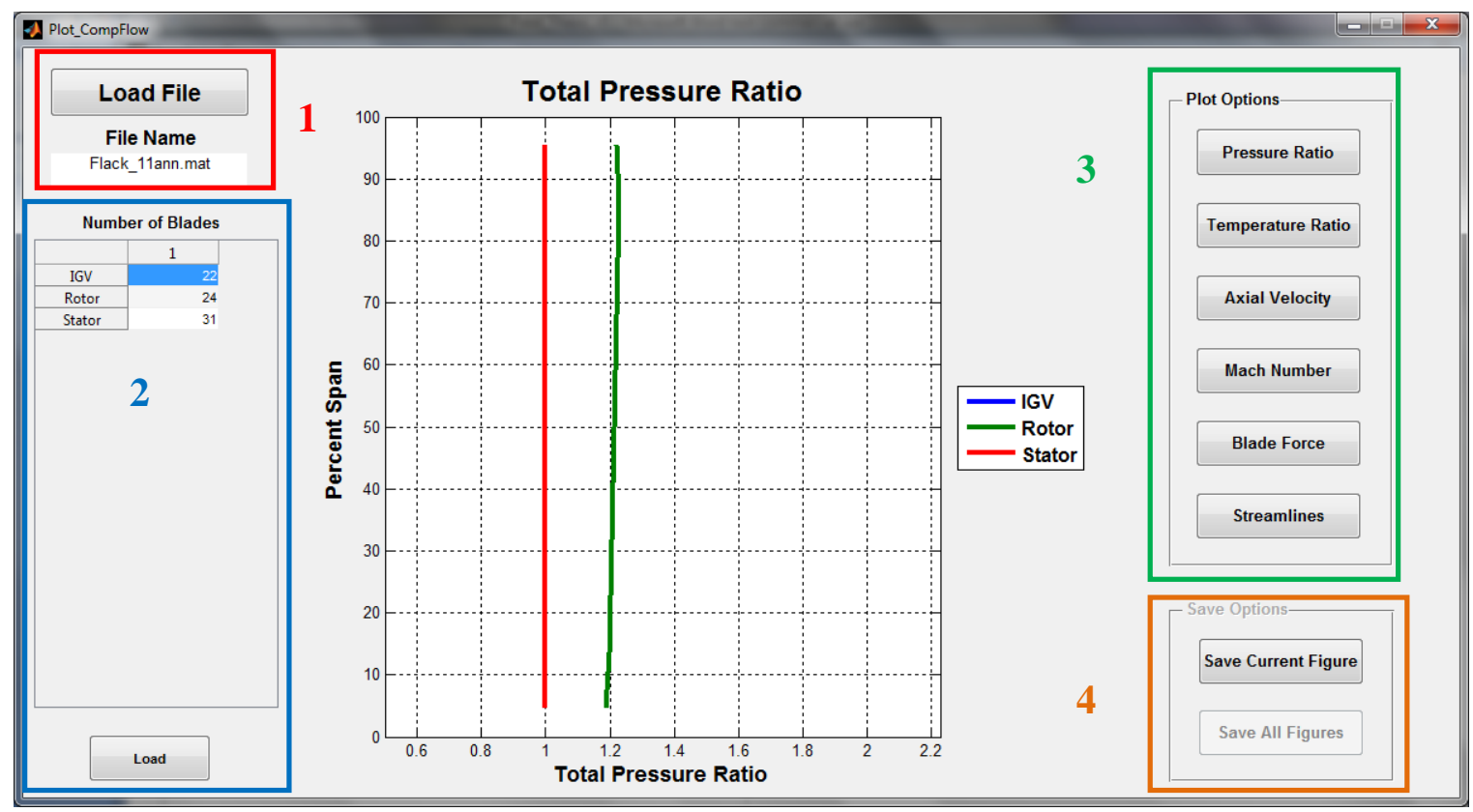

Figure C.5: Window for plotting results from CompFlow 